\title{
Studies in Chlorin Chemistry. III. A Practical Synthesis of C,D-Ring Symmetric Chlorins of Potential Utility in Photodynamic Therapy
}

\author{
William G. O’Neal, William P. Roberts, Indranath Ghosh, Hui Wang and Peter A. Jacobi*
}

Burke Chemical Laboratory, Dartmouth College,Hanover, New Hampshire 03755

Supporting Information

\section{Table of Contents}

\begin{tabular}{|c|c|c|c|c|c|}
\hline Contents & Page & Contents & Page & Contents & Page \\
\hline General Experimental & S2 & Experimental 8be & S14 & ${ }^{1} \mathrm{H} /{ }^{/ 3} \mathrm{C}$ NMR spectra $\mathbf{6 b}$ & S35 \\
\hline Experimental 13a & $\mathrm{S} 2,5$ & Experimental 8ca & S14 & ${ }^{1} \mathrm{H} /{ }^{13} \mathrm{C}$ NMR spectra $\mathbf{6 c}$ & S36 \\
\hline Experimental 18a & $\mathrm{S} 2$ & Experimental 8cb & S14 & ${ }^{1} \mathrm{H} /{ }^{/ 3} \mathrm{C}$ NMR spectra $\mathbf{6 d}$ & S37 \\
\hline Experimental 13b & S3 & Experimental 8cc & S14 & ${ }^{1} \mathrm{H} /{ }^{13} \mathrm{C}$ NMR spectra $\mathbf{6 e}$ & S38 \\
\hline Experimental 13c & $\mathrm{S} 3,5$ & Experimental 8cd & S15 & ${ }^{1} \mathrm{H} /{ }^{13} \mathrm{C}$ NMR spectra $17 \mathbf{a}$ & S39 \\
\hline Experimental 13d & S3,6 & Experimental 8ce & S15 & ${ }^{1} \mathrm{H} /{ }^{13} \mathrm{C}$ NMR spectra $\mathbf{2 4 c B}$ & S40 \\
\hline Experimental 13e & S3 & Experimental 8da & S15 & ${ }^{1} \mathrm{H} /{ }^{13} \mathrm{C}$ NMR spectra 25 & S46 \\
\hline Experimental 22a & S4 & Experimental 8db & S16 & ${ }^{1} \mathrm{H} /{ }^{13} \mathrm{C}$ NMR spectra $28 b$ & S47 \\
\hline Experimental 22c & S4 & Experimental 8dc & S16 & ${ }^{1} \mathrm{H} /{ }^{13} \mathrm{C}$ NMR spectra $8 \mathrm{ac}$ & S48 \\
\hline Experimental 22d & S4 & Experimental 8dd & S16 & ${ }^{1} \mathrm{H} /{ }^{13} \mathrm{C}$ NMR spectra 8ad & S49 \\
\hline Experimental 24a & S4 & Experimental 8de & S17 & ${ }^{1} \mathrm{H} /{ }^{13} \mathrm{C}$ NMR spectra $8 \mathbf{a e}$ & S50 \\
\hline Experimental 24c & S5 & Experimental 8ea & S17 & ${ }^{1} \mathrm{H} /{ }^{13} \mathrm{C}$ NMR spectra $8 b c$ & S51 \\
\hline Experimental 24d & S5 & Experimental 8eb & S17 & ${ }^{1} \mathrm{H} /{ }^{13} \mathrm{C}$ NMR spectra 8 bd & S52 \\
\hline Experimental 27c & S5 & Experimental 8ec & S18 & ${ }^{1} \mathrm{H} /{ }^{13} \mathrm{C}$ NMR spectra $8 \mathbf{b e}$ & $\mathrm{S} 53$ \\
\hline Experimental 25d & S6 & Experimental 8ed & $\mathrm{S} 18$ & ${ }^{1} \mathrm{H} /{ }^{13} \mathrm{C}$ NMR spectra $8 c c$ & S54 \\
\hline Experimental 6a & S6 & Experimental 8ee & $\mathrm{S} 18$ & ${ }^{1} \mathrm{H} /{ }^{13} \mathrm{C}$ NMR spectra $8 \mathrm{~cd}$ & S55 \\
\hline Experimental $\mathbf{6 c}$ & S6 & References & S19 & ${ }^{1} \mathrm{H} /{ }^{13} \mathrm{C}$ NMR spectra 8ce & S56 \\
\hline Experimental 6d & S6 & ${ }^{1} \mathrm{H} /{ }^{13} \mathrm{C}$ NMR spectra 18a & S20 & ${ }^{1} \mathrm{H} /{ }^{13} \mathrm{C}$ NMR spectra 8da & S57 \\
\hline Experimental $\mathbf{6 e}$ & S7 & ${ }^{1} \mathrm{H} /{ }^{13} \mathrm{C}$ NMR spectra 13c & $\mathrm{S} 21$ & ${ }^{1} \mathrm{H} /{ }^{13} \mathrm{C}$ NMR spectra 8db & S58 \\
\hline Experimental 17a & S7 & ${ }^{1} \mathrm{H} /{ }^{13} \mathrm{C}$ NMR spectra $\mathbf{1 3 d}$ & S22 & ${ }^{1} \mathrm{H} /{ }^{13} \mathrm{C}$ NMR spectra $8 d c$ & S59 \\
\hline Experimental $\mathbf{2 4 c B}$ & S7 & ${ }^{1} \mathrm{H} /{ }^{13} \mathrm{C}$ NMR spectra $13 \mathrm{e}$ & $\mathrm{S} 23$ & ${ }^{1} \mathrm{H} /{ }^{13} \mathrm{C}$ NMR spectra 8dd & $\mathrm{S} 60$ \\
\hline Experimental 25 & S8 & ${ }^{1} \mathrm{H} /{ }^{13} \mathrm{C}$ NMR spectra 22a & S24 & ${ }^{1} \mathrm{H} /{ }^{13} \mathrm{C}$ NMR spectra $8 d e$ & S61 \\
\hline Experimental for C,D-rings & S8-11 & ${ }^{1} \mathrm{H} /{ }^{13} \mathrm{C}$ NMR spectra 22b & $\mathrm{S} 25$ & ${ }^{1} \mathrm{H} /{ }^{13} \mathrm{C}$ NMR spectra 8ea & S62 \\
\hline Experimental 28b & S11 & ${ }^{1} \mathrm{H} /{ }^{13} \mathrm{C}$ NMR spectra 22c & S26 & ${ }^{1} \mathrm{H} /{ }^{13} \mathrm{C}$ NMR spectra $8 \mathbf{e b}$ & S63 \\
\hline Experimental 8aa & $\mathrm{S} 12$ & ${ }^{1} \mathrm{H} /{ }^{13} \mathrm{C}$ NMR spectra 22d & S27 & ${ }^{1} \mathrm{H} /{ }^{13} \mathrm{C}$ NMR spectra 8ec & S64 \\
\hline Experimental 8ab & $\mathrm{S} 12$ & ${ }^{1} \mathrm{H} /{ }^{13} \mathrm{C}$ NMR spectra $24 a$ & $\mathrm{~S} 28$ & ${ }^{1} \mathrm{H} /{ }^{13} \mathrm{C}$ NMR spectra 8ed & S65 \\
\hline Experimental 8ac & $\mathrm{S} 12$ & ${ }^{1} \mathrm{H} /{ }^{13} \mathrm{C}$ NMR spectra $24 \mathbf{b}$ & $\mathrm{S} 29$ & ${ }^{1} \mathrm{H} /{ }^{13} \mathrm{C}$ NMR spectra $8 \mathbf{e e}$ & S41-45 \\
\hline Experimental 8ad & S13 & ${ }^{1} \mathrm{H} /{ }^{13} \mathrm{C}$ NMR spectra $24 \mathrm{c}$ & $\mathrm{S} 30$ & Spectra for C,D-rings & S66-68 \\
\hline Experimental 8ae & $\mathrm{S} 13$ & ${ }^{1} \mathrm{H} /{ }^{13} \mathrm{C}$ NMR spectra $24 d$ & S31 & $\mathrm{X}$-ray data for $\mathbf{2 4 c B}$ & \\
\hline Experimental 8ba & $\mathrm{S} 13$ & ${ }^{1} \mathrm{H} /{ }^{13} \mathrm{C}$ NMR spectra $\mathbf{1 3 b}$ & $\mathrm{S} 32$ & & \\
\hline Experimental 8bc & $\mathrm{S} 13$ & ${ }^{1} \mathrm{H} /{ }^{13} \mathrm{C}$ NMR spectra $27 \mathrm{c}$ & $\mathrm{S} 33$ & & \\
\hline Experimental 8bd & S14 & ${ }^{1} \mathrm{H} /{ }^{13} \mathrm{C}$ NMR spectra $\mathbf{6 a}$ & S34 & & \\
\hline
\end{tabular}


I. General: All reactions were performed in flame-dried glassware fitted with rubber septa under positive pressure of nitrogen or argon, unless otherwise noted. Tetrahydrofuran was passed through activated alumina under positive pressure of nitrogen prior to use. Triethylamine was distilled from calcium hydride. Acetonitrile (anhydrous, extra dry), and dimethylformamide (99.8\%, anhydrous) were used as received. TLC was performed on pre-coated $250 \mu \mathrm{m}$ silica 60 $\mathrm{F}_{254}$ glass-backed plates. Flash chromatography was performed using Rf grade silica (60, , 200400 mesh) or neutral alumina (Grade III, $58 \AA, 150$ mesh). Melting points are uncorrected. Infrared spectra were recorded on $\mathrm{NaCl}$ plates either neat or as thin films prepared with $\mathrm{CHCl}_{3}$. ${ }^{1} \mathrm{H}$ and ${ }^{13} \mathrm{C}$ NMR spectra were recorded at 300 or $500 \mathrm{MHz}$. Unless otherwise stated, $\mathrm{CDCl}_{3}$ was used as the solvent. Resonances are reported in parts per million downfield from TMS and were referenced to either the residual solvent peak $\left({ }^{1} \mathrm{H} ; \mathrm{CHCl}_{3}\right.$ : 87.27$)$ or the solvent resonances $\left({ }^{13} \mathrm{C}\right.$; $\left.\mathrm{CDCl}_{3}: \delta 77.0\right)$.

\section{Preparation of formyldihydrodipyrrins 13:}

Method A: 13 from 15

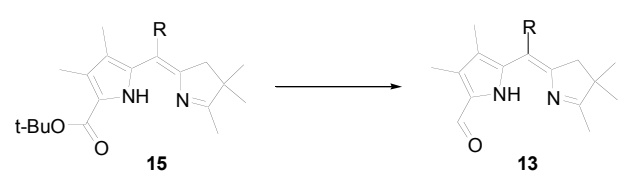

3,4-Dimethyl-5-[phenyl-(4,4,5-trimethyl-3,4-dihydro-pyrrol-2-ylidene)-methyl]-1H-pyrrole2-carbaldehyde (13a). Conditions $i$. TFA $(0.31 \mathrm{~mL}, 4.0 \mathrm{mmol})$ was added to $15 \mathrm{a}(78.5 \mathrm{mg}, 0.20$ $\mathrm{mmol}$ ), and the solution was stirred at $\mathrm{rt}$ for $10 \mathrm{~min}$. The reaction was cooled to $-10^{\circ} \mathrm{C}$ for $10 \mathrm{~min}$ and then treated with triethylorthoformate $(82 \mu \mathrm{l}, 0.50 \mathrm{mmol})$. After $20 \mathrm{~min}$, the mixture was poured into $10 \%$ aq $\mathrm{KH}_{2} \mathrm{PO}_{4}(10 \mathrm{~mL})$ at $0{ }^{\circ} \mathrm{C}$ and the solution extracted with $\mathrm{CH}_{2} \mathrm{Cl}_{2}(3 \times 5 \mathrm{~mL})$. The combined organic extracts were washed sequentially with saturated aq $\mathrm{NaHCO}_{3}$ and brine, dried over $\mathrm{Na}_{2} \mathrm{SO}_{4}$, filtered, and concentrated. The residue was purified by flash chromatography (silica gel, gradient elution: EtOAc:hexanes $=1: 9$ to $4: 6)$ to give 13a $(33.4 \mathrm{mg}, 52 \%)$ as a solid that was identical to the literature compound. ${ }^{1}$ Also isolated was side product 18a $(10.0 \mathrm{mg}$, 13\%):1-\{3,4-Dimethyl-5-[phenyl-(4,4,5-trimethyl-3,4-dihydro-pyrrol-2-ylidene)-methyl]-1Hpyrrol-2-yl\}-2,2,2-trifluoro-ethanone (18a). $\mathrm{R}_{f}(3: 7$ EtOAc/hexanes) 0.64 ; $\operatorname{IR}($ thin film) 1652, 1581, 1343, 1191, $1132 \mathrm{~cm}^{-1} ;{ }^{1} \mathrm{H}$ NMR $\left(500 \mathrm{MHz}, \mathrm{CDCl}_{3}\right) \delta 1.17(\mathrm{~s}, 6 \mathrm{H}), 1.22(\mathrm{~s}, 3 \mathrm{H}), 2.22(\mathrm{~s}$, $3 \mathrm{H}), 2.30(\mathrm{~s}, 3 \mathrm{H}), 2.38(\mathrm{~s}, 2 \mathrm{H}), 7.23-7.25(\mathrm{~m}, 2 \mathrm{H}), 7.37-7.43(\mathrm{~m}, 3 \mathrm{H}), 12.76(\mathrm{br} \mathrm{s}, 1 \mathrm{H}) ;{ }^{13} \mathrm{C} \mathrm{NMR}$ $\left(500 \mathrm{MHz}, \mathrm{CDCl}_{3}\right) \delta 10.0,11.3,16.1,26.0,44.6,48.5,116.8,119.1,120.0,122.2,122.4,127.8$, 129.0, 129.9, 137.3, 139.0, 154.2, 167.9 (q), 190.9. ${ }^{19} \mathrm{~F}\left(300 \mathrm{MHz}, \mathrm{CDCl}_{3}\right) \delta$-72.9. HRMS (EI) Calcd for $\mathrm{C}_{22} \mathrm{H}_{23} \mathrm{~F}_{3} \mathrm{~N}_{2} \mathrm{O}$ : 388.1762; found: 388.1762 .

3,4-Dimethyl-5-[phenyl-(4,4,5-trimethyl-3,4-dihydro-pyrrol-2-ylidene)-methyl]-1H-pyrrole2-carbaldehyde (13a). Conditions ii. $30 \% \mathrm{HBr}$ in $\mathrm{HOAc}(620 \mu \mathrm{L}, 3.10 \mathrm{mmol})$ was added to $15 \mathrm{a}$ $(122 \mathrm{mg}, 0.310 \mathrm{mmol})$, and the solution was stirred at $\mathrm{rt}$ for $1 \mathrm{~h}$. The mixture was then added to $10 \% \mathrm{KH}_{2} \mathrm{PO}_{4}(5 \mathrm{~mL})$ at $0^{\circ} \mathrm{C}$ and extracted with $\mathrm{CH}_{2} \mathrm{Cl}_{2}(3 \times 5 \mathrm{~mL})$. The combined organic extracts were washed sequentially with saturated aq $\mathrm{NaHCO}_{3}$ and brine, dried over $\mathrm{Na}_{2} \mathrm{SO}_{4}$, filtered, and concentrated. The resulting oil was dissolved in $\mathrm{CH}_{2} \mathrm{Cl}_{2}(2.0 \mathrm{~mL})$ and 
triethylorthoformate $(130 \mu \mathrm{L}, 0.775 \mathrm{mmol})$. The mixture was then added to TFA (460 $\mu \mathrm{L}, 6.20$ $\mathrm{mmol})$ at $-10^{\circ} \mathrm{C}$. After stirring for 20 minutes, the mixture was added to $10 \% \mathrm{KH}_{2} \mathrm{PO}_{4}(10 \mathrm{~mL})$ at $0^{\circ} \mathrm{C}$ and the resulting solution extracted with $\mathrm{CH}_{2} \mathrm{Cl}_{2}(3 \times 10 \mathrm{~mL})$. The combined organic layers were washed sequentially with saturated aq $\mathrm{NaHCO}_{3}$ and brine, dried over $\mathrm{Na}_{2} \mathrm{SO}_{4}$, filtered, and concentrated. The resulting oil was purified by flash chromatography (silica gel, gradient elution: EtOAc:hexanes $=1: 9$ to $4: 6)$ to give 13a $(63.2 \mathrm{mg}, 64 \%)$, which was identical to the literature compound $^{1}$ and the material prepared by Conditions $i$.

3,4-Dimethyl-5-[1-(4,4,5-trimethyl-3,4-dihydro-pyrrol-2-ylidene)-ethyl]-1H-pyrrole-2-carbaldehyde (13b). Following Conditions $i i$ outlined for 13a, reaction of $\mathbf{1 5 b}(914 \mathrm{mg}, 2.77 \mathrm{mmol})$ with $30 \% \mathrm{HBr}$ in $\mathrm{HOAc}(5.52 \mathrm{~mL}, 27.7 \mathrm{mmol})$, TFA $(4.11 \mathrm{~mL}, 55.3 \mathrm{mmol})$, triethylorthoformate (1.14 mL, $6.93 \mathrm{mmol})$, and $\mathrm{CH}_{2} \mathrm{Cl}_{2}(7.5 \mathrm{~mL})$ gave $13 \mathbf{b}(177 \mathrm{mg}, 25 \%)$ that was identical to the literature compound. ${ }^{1}$ Conditions $i$ were previously used to prepare $13 \mathbf{b}$ in $32 \%$ yield. ${ }^{1}$

3,4-Dimethyl-5-(4,4,5-trimethyl-3,4-dihydro-pyrrol-2-ylidenemethyl)-1Hpyrrole-2-carbaldehyde (13c). Following Conditions $i$ outlined for 13a, reaction of $15 \mathbf{c}(408 \mathrm{mg}, 1.29 \mathrm{mmol})$ with TFA $(1.9 \mathrm{~mL}, 25.6 \mathrm{mmol})$ and triethylorthoformate $(0.54 \mathrm{~mL}, 3.3 \mathrm{mmol})$ gave $13 \mathbf{c}(223 \mathrm{mg}$, $71 \%$ ) as a yellow crystalline solid, $\mathrm{mp} 158.5-159.2{ }^{\circ} \mathrm{C} . \mathrm{R}_{f}(2: 5 \mathrm{EtOAc} /$ hexanes $) 0.43$; IR(thin film) 3356, $1634 \mathrm{~cm}^{-1}$; ${ }^{1} \mathrm{H}$ NMR $\left(500 \mathrm{MHz}, \mathrm{CDCl}_{3}\right) \delta 1.15(\mathrm{~s}, 6 \mathrm{H}), 1.96(\mathrm{~s}, 3 \mathrm{H}), 2.16(\mathrm{~s}, 3 \mathrm{H}), 2.32(\mathrm{~s}$, $3 \mathrm{H}), 2.56(\mathrm{~d}, J=1.71,2 \mathrm{H}), 5.82(\mathrm{~s}, 1 \mathrm{H}), 9.53(\mathrm{~s}, 1 \mathrm{H}), 11.11($ br s, $1 \mathrm{H}) ;{ }^{13} \mathrm{C}$ NMR $(500 \mathrm{MHz}$, $\left.\mathrm{CDCl}_{3}\right) \delta 8.4,8.8,15.9,25.6,44.5,48.6,102.7,118.8,128.9,131.0,135.4,154.6,175.7,190.3$. Anal. Calcd for $\mathrm{C}_{15} \mathrm{H}_{20} \mathrm{~N}_{2} \mathrm{O}$ : C, 73.74; H, 8.25; N, 11.47; O, 6.55. Found: C, 73.67; H, 8.27; N, $11.38 .^{1}$

3,4-Dimethyl-5-[1-(4,4,5-trimethyl-3,4-dihydro-pyrrol-2-ylidene)-hexyl]-1Hpyrrole-2-carbaldehyde (13d). Following Conditions ii outlined for 13a, reaction of 15d $(289 \mathrm{mg}, 0.748 \mathrm{mmol})$ with $30 \% \mathrm{HBr}$ in $\mathrm{HOAc}(1.5 \mathrm{~mL}, 7.5 \mathrm{mmol})$, TFA $(1.1 \mathrm{~mL}, 15 \mathrm{mmol})$, triethylorthoformate (307 $\mu \mathrm{L}, 1.86 \mathrm{mmol})$, and $\mathrm{CH}_{2} \mathrm{Cl}_{2}(1.1 \mathrm{~mL})$ gave $13 \mathrm{~d}(133 \mathrm{mg}, 57 \%)$ as a yellow oil after flash chromatography (silica gel, EtOAc:hexanes $=2: 5) . \quad \mathrm{R}_{f}(2: 5 \mathrm{EtOAc} /$ hexanes) 0.26 ; IR(thin film) $3243,1639 \mathrm{~cm}^{-1}$; ${ }^{1} \mathrm{H}$ NMR $\left(500 \mathrm{MHz}, \mathrm{CDCl}_{3}\right) \delta 0.89$ (t, $\left.J=7.1,3 \mathrm{H}\right), 1.89(\mathrm{~s}, 6 \mathrm{H}), 1.32(\mathrm{~m}, 4 \mathrm{H})$, $1.42(\mathrm{~m}, 2 \mathrm{H}), 2.09(\mathrm{~s}, 3 \mathrm{H}), 2.14(\mathrm{~s}, 3 \mathrm{H}), 2.90(\mathrm{~s}, 3 \mathrm{H}), 2.42(\mathrm{~m}, 2 \mathrm{H}) 2.59(\mathrm{~s}, 2 \mathrm{H}), 9.56(\mathrm{~s}, 1 \mathrm{H})$, 11.46 (br s, 1H); ${ }^{13} \mathrm{C}$ NMR $\left(500 \mathrm{MHz}, \mathrm{CDCl}_{3}\right) \delta$ 9.1, 10.7, 14.3, 16.0, 22.8, 25.8, 26.1, 29.2, 31.0, $32.0,43.6,48.1,118.8,119.0,128.6,136.4,152.0,176.3,187.6$. HRMS (EI) Calcd for $\mathrm{C}_{20} \mathrm{H}_{30} \mathrm{~N}_{2} \mathrm{O}: 314.2358$; found: 314.2360 .

\section{3,4-Dimethyl-5-[1-(4,4,5-trimethyl-3,4-dihydro-pyrrol-2-ylidene)-undecyl]-1 $H$-pyrrole-2-}

carbaldehyde (13e). Following Conditions ii outlined for 13a, reaction of 15e (135 mg, 0.296 mmol) with $30 \% \mathrm{HBr}$ in $\mathrm{HOAc}(0.59 \mathrm{~mL}, 3.0 \mathrm{mmol})$, TFA $(0.44 \mathrm{~mL}, 6.0 \mathrm{mmol})$, triethylorthoformate $(120 \mu \mathrm{L}, 0.729 \mathrm{mmol})$, and $\mathrm{CH}_{2} \mathrm{Cl}_{2}(1.0 \mathrm{~mL})$ gave $13 \mathrm{e}(69.4 \mathrm{mg}, 61 \%)$ as a yellow oil after flash chromatography (silica gel, EtOAc:hexanes $=2: 5) . \mathrm{R}_{f}(2: 5 \mathrm{EtOAc} /$ hexanes $)$ 0.37; IR(thin film) 3244, $1636 \mathrm{~cm}^{-1} ;{ }^{1} \mathrm{H}$ NMR $\left(500 \mathrm{MHz}, \mathrm{CDCl}_{3}\right) \delta 0.89(\mathrm{t}, J=7.08,3 \mathrm{H}), 1.2(\mathrm{~s}$, $6 \mathrm{H}), 1.36-1.22(\mathrm{~m}, 14 \mathrm{H}), 1.44-1.38(\mathrm{~m}, 2 \mathrm{H}), 2.1(\mathrm{~s}, 3 \mathrm{H}), 2.14(\mathrm{~s}, 3 \mathrm{H}), 2.27(\mathrm{~s}, 3 \mathrm{H}), 2.43(\mathrm{~m}, 2 \mathrm{H})$, $2.61(\mathrm{~s}, 2 \mathrm{H}), 9.58(\mathrm{~s}, 1 \mathrm{H}), 11.42(\mathrm{br} \mathrm{s}, 1 \mathrm{H}) ;{ }^{13} \mathrm{C}$ NMR $\left(500 \mathrm{MHz}, \mathrm{CDCl}_{3}\right) \delta$ 9.1, 10.8, 14.3, 16.0, 22.9, 25.8, 26.1, 29.49, 29.50, 29.71, 29.76, 29.77, 29.82, 31.1, 32.1, 43.6, 48.1, 118.8, 119.1, 128.6, 136.5, 152.1, 176.3, 187.6. HRMS (EI) Calcd for $\mathrm{C}_{25} \mathrm{H}_{40} \mathrm{~N}_{2} \mathrm{O}: 384.3141$; found: 384.3138 . 
Method B: 13 from 22

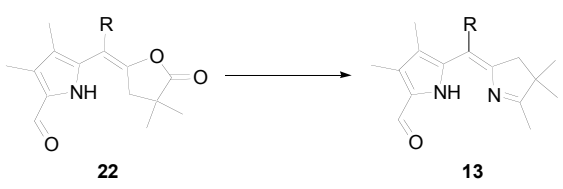

5-[(4,4-Dimethyl-5-oxo-dihydro-furan-2-ylidene)-phenyl-methyl]-3,4-dimethyl-1H-pyrrole-2carbaldehyde (22a). TFA (3.76 mL, $50.6 \mathrm{mmol})$ was added to $11 \mathrm{a}(500 \mathrm{mg}, 1.26 \mathrm{mmol})$, and the solution was stirred vigorously for $5 \mathrm{~min}$. The reaction was cooled to $0^{\circ} \mathrm{C}$ for $10 \mathrm{~min}$ and then treated with triethylorthoformate $(1.14 \mathrm{~mL}, 6.93 \mathrm{mmol})$. After $20 \mathrm{~min}$, the reaction was poured into $10 \%$ aq $\mathrm{KH}_{2} \mathrm{PO}_{4}(20 \mathrm{ml})$ at $0^{\circ} \mathrm{C}$ and extracted with $\mathrm{CH}_{2} \mathrm{Cl}_{2}(3 \times 10 \mathrm{ml})$. The combined organic extracts were washed sequentially with water and saturated aq $\mathrm{NaHCO}_{3}$, dried over $\mathrm{Na}_{2} \mathrm{SO}_{4}$, filtered, and concentrated. The residue was purified by flash chromatography (silica gel, EtOAc:hexanes $=3: 7)$ to give 22a $(361 \mathrm{mg}, 87 \%)$ as a colorless crystalline solid, $\mathrm{mp}$ 186.6$188.0^{\circ} \mathrm{C} . \mathrm{R}_{f}\left(4: 6 \mathrm{EtOAc} /\right.$ hexanes) 0.36 ; IR(thin film) $3239,1799,1628,1442,1232,1067 \mathrm{~cm}^{-1} ;{ }^{1} \mathrm{H}$ NMR $\left(500 \mathrm{MHz}, \mathrm{CDCl}_{3}\right) \delta 1.35(\mathrm{~s}, 6 \mathrm{H}), 1.84(\mathrm{~s}, 3 \mathrm{H}), 2.28(\mathrm{~s}, 3 \mathrm{H}), 2.72(\mathrm{~s}, 2 \mathrm{H}), 7.21(\mathrm{~m}, 1 \mathrm{H})$, $7.28(\mathrm{~m}, 4 \mathrm{H}), 9.48(\mathrm{~s}, 1 \mathrm{H}), 9.63(\mathrm{br} \mathrm{s}, 1 \mathrm{H}) ;{ }^{13} \mathrm{C} \mathrm{NMR}\left(500 \mathrm{MHz}, \mathrm{CDCl}_{3}\right) \delta$ 9.3, 9.4, 25.1, 39.8, 41.7, 109.5, 120.8, 127.6, 128.5, 128.8, 129.5, 132.2, 134.7, 135.6, 147.8, 177.3, 179.9. Anal. Calcd for $\mathrm{C}_{20} \mathrm{H}_{21} \mathrm{NO}_{3}: \mathrm{C}, 74.28 ; \mathrm{H}, 6.55 ; \mathrm{N}, 4.33 ; \mathrm{O}, 14.84$. Found: $\mathrm{C}, 74.11 ; \mathrm{H}, 6.55 ; \mathrm{N}, 4.34$.

5-(4,4-Dimethyl-5-oxo-dihydro-furan-2-ylidenemethyl)-3,4-dimethyl-1 Hpyrrole-2-carbaldehyde (22c). Following the procedure outlined for 22a, reaction of 11c $(160 \mathrm{mg}, 0.50 \mathrm{mmol})$ with TFA $(1.5 \mathrm{~mL}, 20 \mathrm{mmol})$ and triethylorthoformate $(450 \mu \mathrm{L}, 2.7 \mathrm{mmol})$ gave $22 \mathrm{c}(119 \mathrm{mg}$, $95 \%$ ) as a colorless solid, $166.8-167.5^{\circ} \mathrm{C} . \mathrm{R}_{f}(2: 5 \mathrm{EtOAc} /$ hexanes) 0.28 ; IR(thin film) 3297,1795 , $1626 \mathrm{~cm}^{-1}$; ${ }^{1} \mathrm{H}$ NMR $\left(500 \mathrm{MHz}, \mathrm{CDCl}_{3}\right) \delta 1.38(\mathrm{~s}, 6 \mathrm{H}), 1.99(\mathrm{~s} 3 \mathrm{H}), 2.28(\mathrm{~s}, 3 \mathrm{H}) 3.01(\mathrm{~s}, 2 \mathrm{H})$, $6.23(\mathrm{~s}, 1 \mathrm{H}), 8.50(\mathrm{br} \mathrm{s}, 1 \mathrm{H}), 9.58(\mathrm{~s}, 1 \mathrm{H}) ;{ }^{13} \mathrm{C} \mathrm{NMR}\left(500 \mathrm{MHz}, \mathrm{CDCl}_{3}\right) \delta 8.86,8.90,25.2,40.2$, 40.4, 96.6, 121.1, 129.6, 132.0, 132.4, 150.3, 176.4, 179.2. Anal. Calcd for $\mathrm{C}_{14} \mathrm{H}_{17} \mathrm{NO}_{3}$ : C, 68.00; H, 6.93; N, 5.66; O, 19.41. Found: C, 67.94; H, 6.96; N, 5.63.

\section{5-[1-(4,4-Dimethyl-5-oxo-dihydro-furan-2-ylidene)-hexyl]-3,4-dimethyl-1 Hpyrrole-2-carb-}

aldehyde (22d). Following the procedure outlined for 22a, reaction of $11 d(94 \mathrm{mg}, 0.24 \mathrm{mmol}$ ) with TFA $(0.73 \mathrm{~mL}, 9.7 \mathrm{mmol})$ and triethylorthoformate $(220 \mu \mathrm{L}, 1.3 \mathrm{mmol})$ gave $22 \mathrm{~d}(64.7 \mathrm{mg}$, $85 \%)$ as a colorless oil. $\mathrm{R}_{f}\left(1: 5 \mathrm{EtOAc} /\right.$ hexanes) 0.20 ; IR(neat) $3246,1799,1628 \mathrm{~cm}^{-1}$; ${ }^{1} \mathrm{H}$ NMR $\left(300 \mathrm{MHz}, \mathrm{CDCl}_{3}\right) 0.85$ (t, J=6.7, 3H), $1.25(\mathrm{~m}, 6 \mathrm{H}), 1.29(\mathrm{~s}, 6 \mathrm{H}), 1.91(\mathrm{~s}, 3 \mathrm{H}), 2.29(\mathrm{~s}, 3 \mathrm{H}), 2.44$ $(\mathrm{t}, J=6.8,2 \mathrm{H}), 2.53(\mathrm{~s}, 2 \mathrm{H}), 8.92(\mathrm{br} \mathrm{s}, 1 \mathrm{H}), 9.58(\mathrm{~s}, 1 \mathrm{H}) ;{ }^{13} \mathrm{C} \mathrm{NMR}\left(300 \mathrm{MHz}, \mathrm{CDCl}_{3}\right) \delta 9.1,9.5$, 14.2, 22.6, 24.9, 28.0, 30.1, 31.6, 40.3, 40.4, 110.0, 119.7, 129.2, 132.2, 135.2, 146.6, 176.9, 180.0. HRMS (EI) Calcd for $\mathrm{C}_{19} \mathrm{H}_{27} \mathrm{NO}_{3}: 317.1991$; found: 317.1995 .

5-(4,4-Dimethyl-2,5-dioxo-1-phenyl-hexyl)-3,4-dimethyl-1H-pyrrole-2-carbaldehyde (24a). A solution of 22a $(389 \mathrm{mg}, 1.20 \mathrm{mmol})$ in THF $(24 \mathrm{~mL})$ was cooled to $-78^{\circ} \mathrm{C}$ and treated dropwise with $1.6 \mathrm{M} \mathrm{MeLi}$ in $\mathrm{Et}_{2} \mathrm{O}(1.50 \mathrm{~mL}, 2.41 \mathrm{mmol})$ over $20 \mathrm{~min}$. The reaction was then quenched by addition to $60 \mathrm{~mL}$ of saturated aq $\mathrm{NH}_{4} \mathrm{Cl}$. After allowing the mixture to warm to rt, $\mathrm{Et}_{2} \mathrm{O}(10 \mathrm{~mL})$ was added and the layers were separated. The organic layer was washed sequentially with water and brine, dried over $\mathrm{Na}_{2} \mathrm{SO}_{4}$, filtered and concentrated. The resulting yellow oil was purified by flash chromatography (silica gel, EtOAc:hexanes $=3: 7)$ to give $\mathbf{2 4 a}(318 \mathrm{mg}, 78 \%)$ as a colorless crystalline solid, mp $130.2-131.4^{\circ} \mathrm{C} . \mathrm{R}_{f}(2: 3 \mathrm{EtOAc} /$ hexanes) 0.21 ; IR(thin film) $3427,1704,1834$, 
$1057 \mathrm{~cm}^{-1} ;{ }^{1} \mathrm{H}$ NMR $\left(500 \mathrm{MHz}, \mathrm{CDCl}_{3}\right) 1.135(\mathrm{~s}, 3 \mathrm{H}), 1.141(\mathrm{~s}, 3 \mathrm{H}), 1.87(\mathrm{~s}, 3 \mathrm{H}), 2.17(\mathrm{~s}, 3 \mathrm{H})$, $2.22(\mathrm{~s}, 3 \mathrm{H}), 2.73(\mathrm{~d}, \mathrm{~J}=17.8,1 \mathrm{H}), 2.95(\mathrm{~d}, \mathrm{~J}=17.8,1 \mathrm{H}), 5.15(\mathrm{~s}, 1 \mathrm{H}), 7.23(\mathrm{~d}, \mathrm{~J}=7.32,2 \mathrm{H}), 7.30$ $(\mathrm{m}, 1 \mathrm{H}), 7.36(\mathrm{~m}, 2 \mathrm{H}), 9.54(\mathrm{~s}, 1 \mathrm{H}), 9.65(\mathrm{br} \mathrm{s}, 1 \mathrm{H}) ;{ }^{13} \mathrm{C} \mathrm{NMR}\left(500 \mathrm{MHz}, \mathrm{CDCl}_{3}\right) \delta 8.8,9.0,24.8$, 25.6, 25.9, 46.3, 52.3, 55.3, 118.8, 128.3, 128.70, 128.72, 129.7, 131.6, 134.2, 135.7, 176.9, 206.0, 213.3. Anal. Calcd for $\mathrm{C}_{21} \mathrm{H}_{25} \mathrm{NO}_{3}$ : C, 74.31; H, 7.42; N, 4.13; O, 14.14. Found: C, 74.18; H, 7.42; N, 4.17.

5-(4,4-Dimethyl-2,5-dioxo-hexyl)-3,4-dimethyl-1 $H$-pyrrole-2-carbaldehyde (24c). Following the procedure outlined for $\mathbf{2 4 a}$, reaction of $22 \mathrm{c}(265 \mathrm{mg}, 1.07 \mathrm{mmol})$ in THF $(53 \mathrm{~mL})$ with $1.6 \mathrm{M}$ MeLi in $\mathrm{Et}_{2} \mathrm{O}(1.33 \mathrm{~mL}, 2.14 \mathrm{mmol})$ gave $24 \mathrm{c}(217 \mathrm{mg}, 77 \%)$ after flash chromatography (silica gel, EtOAc:hexanes = 2:5). Colorless crystalline solid, $\mathrm{mp} 104-105^{\circ} \mathrm{C} . \mathrm{R}_{f}(2: 5 \mathrm{EtOAc} / \mathrm{hexanes})$ 0.23; IR(thin film) 3248, 1704, $1627 \mathrm{~cm}^{-1} ;{ }^{1} \mathrm{H}$ NMR (500 MHz, $\left.\mathrm{CDCl}_{3}\right) \delta 1.20$ (s, 6H), 1.91 (s, $3 \mathrm{H}), 2.18(\mathrm{~s}, 3 \mathrm{H}), 2.19(\mathrm{~s}, 3 \mathrm{H}), 2.26(\mathrm{~s}, 3 \mathrm{H}), 2.78(\mathrm{~s}, 2 \mathrm{H}), 3.74(\mathrm{~s}, 2 \mathrm{H}), 9.50(\mathrm{~s}, 1 \mathrm{H}), 10.25$ (br s, $1 \mathrm{H}) ;{ }^{13} \mathrm{C} \mathrm{NMR}\left(500 \mathrm{MHz}, \mathrm{CDCl}_{3}\right) \delta 8.6,9.1,25.5,25.6,40.6,46.2,52.1,119.5,128.8,131.7$, 132.4, 176.7, 204.7, 213.4. HRMS (EI) Calcd for $\mathrm{C}_{15} \mathrm{H}_{21} \mathrm{NO}_{3}$ : 263.1521; found: 263.1525. Anal. Calcd for $\mathrm{C}_{15} \mathrm{H}_{21} \mathrm{NO}_{3}$ : C, 68.42; H, 8.04; N, 5.32; O, 18.23. Found: C, 68.19; H, 8.00; N, 5.27.

5-(4,4-Dimethyl-2,5-dioxo-1-pentyl-hexyl)-3,4-dimethyl-1H-pyrrole-2-carbaldehyde (24d). Following the procedure outlined for $\mathbf{2 4 a}$, reaction of $\mathbf{2 2 d}(65 \mathrm{mg}, 0.20 \mathrm{mmol})$ in THF $(4.0 \mathrm{~mL})$ with $1.6 \mathrm{M} \mathrm{MeLi}$ in $\mathrm{Et}_{2} \mathrm{O}(0.26 \mathrm{~mL}, 0.40 \mathrm{mmol})$ gave $\mathbf{2 4 d}(50 \mathrm{mg}, 74 \%)$ as a clear colorless oil after flash chromatography (silica gel, EtOAc:hexanes $=2: 3) . \quad \mathrm{R}_{\mathrm{f}}(2: 3$ EtOAc/hexanes) 0.33; IR(neat) 3247, 1707, $1627 \mathrm{~cm}^{-1} ;{ }^{1} \mathrm{H}$ NMR (500 MHz, $\left.\mathrm{CDCl}_{3}\right) 0.82(\mathrm{t}, J=6.6,3 \mathrm{H}), 1.11(\mathrm{~s}, 3 \mathrm{H})$, 1.13(s, 3H), $1.22(\mathrm{~m}, 2 \mathrm{H}), 1.66(\mathrm{~m}, 6 \mathrm{H}), 1.94(\mathrm{~s}, 3 \mathrm{H}), 2.16(\mathrm{~s}, 3 \mathrm{H}), 2.24(\mathrm{~s}, 3 \mathrm{H}), 2.58(\mathrm{~d}, J=17.8$, $1 \mathrm{H}), 2.79(\mathrm{~d}, J=17.8,1 \mathrm{H}), 3.76(\mathrm{~d}, t=6.3,1 \mathrm{H}), 9.49(\mathrm{br} \mathrm{s}, 1 \mathrm{H}), 9.52(\mathrm{~s}, 1 \mathrm{H}) ;{ }^{13} \mathrm{C} \mathrm{NMR}(500 \mathrm{MHz}$, $\left.\mathrm{CDCl}_{3}\right) \delta 8.7,9.1,14.1,22.5,25.0,25.6,25.7,27.0,31.2,31.7,45.8,50.0,52.3,119.1,129.0$, 132.0, 134.7, 176.8, 207.3, 213.2; HRMS (EI) Calcd for $\mathrm{C}_{20} \mathrm{H}_{31} \mathrm{NO}_{3}$ : 333.2304; found: 333.2310 .

\section{3,4-Dimethyl-5-[phenyl-(4,4,5-trimethyl-3,4-dihydro-pyrrol-2-ylidene)-methyl]-1H-pyrrole-}

2-carbaldehyde (13a). A solution of $24 a(83.3 \mathrm{mg}, 0.245 \mathrm{mmol})$ in $\mathrm{CH}_{3} \mathrm{CN}(2.7 \mathrm{~mL})$ was treated with $\left(\mathrm{NH}_{4}\right)_{2} \mathrm{CO}_{3}(238 \mathrm{mg}, 2.45 \mathrm{mmol})$ and basic alumina (417 mg, Grade I), flushed with nitrogen, and heated to $75^{\circ} \mathrm{C}$ in a sealed flask for $8 \mathrm{hrs}$. At the end of this period, the mixture was cooled to rt, filtered, and concentrated. The residue was dissolved in $\mathrm{CH}_{2} \mathrm{Cl}_{2}$, washed with water, dried over $\mathrm{Na}_{2} \mathrm{SO}_{4}$, filtered and concentrated. The resulting oil was purified by flash chromatography (silica gel, EtOAc:hexanes = 1:4) to give 13a $(29.4 \mathrm{mg}, 37 \%)$ as a solid that was identical to the literature compound $^{1}$ and that prepared by Method A.

\section{3,4-Dimethyl-5-(4,4,5-trimethyl-3,4-dihydro-pyrrol-2-ylidenemethyl)-1Hpyrrole-2-carb-}

aldehyde (13c). A solution of $24 \mathbf{c}(100 \mathrm{mg}, 0.38 \mathrm{mmol})$ in $\mathrm{CH}_{3} \mathrm{CN}$ (4.2 mL) was treated with $\left(\mathrm{NH}_{4}\right)_{2} \mathrm{CO}_{3}(365 \mathrm{mg}, 3.80 \mathrm{mmol})$ and basic alumina $(500 \mathrm{mg}$, Grade I), flushed with nitrogen, sealed, and stirred at $\mathrm{rt}$ for $24 \mathrm{~h}$. The reaction mixture was then heated to $75^{\circ} \mathrm{C}$ for $1 \mathrm{~h}$. At the end of this period, the mixture was cooled to $\mathrm{rt}$, filtered, and concentrated. The residue was dissolved in $\mathrm{CH}_{2} \mathrm{Cl}_{2}$, washed with water, dried over $\mathrm{Na}_{2} \mathrm{SO}_{4}$, filtered and concentrated. The resulting oil was purified by flash chromatography (silica gel, EtOAc:hexanes $=1: 2)$ to give 13c $(53.8 \mathrm{mg}, 58 \%)$ as a yellow crystalline solid that was identical to the literature compound ${ }^{1}$ and that prepared by Method A. Also isolated was the side product 27c (16 mg, 17\%). Crystalline solid, mp 166-168 ${ }^{\circ} \mathrm{C} . \mathrm{R}_{f}$ (3:2 EtOAc/hexanes) 0.33; IR(thin film) 3256, 1708, $1640 \mathrm{~cm}^{-1} ;{ }^{1} \mathrm{H}$ NMR (500 MHz, $\left.\mathrm{CDCl}_{3}\right) 1.31(\mathrm{~s}, 6 \mathrm{H}), 1.89(\mathrm{~s}, 3 \mathrm{H}), 2.04(\mathrm{~s}, 3 \mathrm{H}), 2.29(\mathrm{~s}, 3 \mathrm{H}), 2.46(\mathrm{~s}, 2 \mathrm{H}), 9.32(\mathrm{br} \mathrm{s}, 1 \mathrm{H}), 9.61$ (s, 
$1 \mathrm{H}) ;{ }^{13} \mathrm{C}$ NMR (500 MHz, $\left.\mathrm{CDCl}_{3}\right) \delta$ 9.1, 10.1, 13.9, 27.3, 42.0, 51.0, 120.5, 128.4, 129.9, 130.9, 131.4, 177.3, 182.4, 205.3; HRMS (EI) Calcd for $\mathrm{C}_{15} \mathrm{H}_{19} \mathrm{NO}_{2}$ : 245.1416; found: 245.1422; Anal. Calcd for $\mathrm{C}_{15} \mathrm{H}_{19} \mathrm{NO}_{2}$ : C, 73.44; H, 7.81; N, 5.71; O, 13.04. Found: C, 73.68; H, 7.81; N, 5.73.

3,4-Dimethyl-5-[1-(4,4,5-trimethyl-3,4-dihydro-pyrrol-2-ylidene)-hexyl]-1Hpyrrole-2-carbaldehyde (13d). Following the procedure outlined for 13a, reaction of $\mathbf{2 4 d}(108 \mathrm{mg}, 0.324 \mathrm{mmol})$ in $\mathrm{CH}_{3} \mathrm{CN}(3.6 \mathrm{~mL})$ with $\left(\mathrm{NH}_{4}\right)_{2} \mathrm{CO}_{3}(311 \mathrm{mg}, 3.20 \mathrm{mmol})$ and basic alumina $(540 \mathrm{mg}$, Grade $\mathrm{I})$ gave 13d (78.4 mg, 77\%) as a yellow oil that was identical to the material prepared by Method A.

\section{Preparation of bis-formyldihydrodipyrrins 6:}

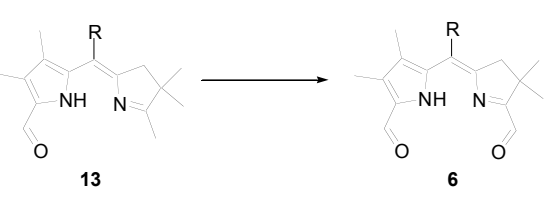

5-[(5-Formyl-4,4-dimethyl-3,4-dihydro-pyrrol-2-ylidene)-phenyl-methyl]-3,4-dimethyl-1Hpyrrole-2-carbaldehyde (6a). A solution of 13a (244 mg, $0.727 \mathrm{mmol})$ in $\mathrm{CH}_{2} \mathrm{Cl}_{2}(15 \mathrm{~mL})$ and pyridine $(59 \mu \mathrm{L}, 0.73 \mathrm{mmol})$ was treated with $\mathrm{SeO}_{2}(83.3 \mathrm{mg}, 0.727 \mathrm{mmol})$ and stirred at $\mathrm{rt}$ for 5 $\mathrm{h}$. The solvent was then removed by rotary evaporation, and the residue was redissolved in DMF $(10 \mathrm{~mL})$ and heated to $80^{\circ} \mathrm{C}$ for $15 \mathrm{~min}$. The reaction mixture was cooled to rt, filtered, and poured into water $(50 \mathrm{~mL})$. The solution was extracted with $\mathrm{CH}_{2} \mathrm{Cl}_{2}(4 \times 20 \mathrm{~mL})$, and the combined organic extracts were washed sequentially with saturated aq $\mathrm{NaHCO}_{3}$ and brine, dried over $\mathrm{Na}_{2} \mathrm{SO}_{4}$, filtered, and concentrated. The resulting oil was purified by flash chromatography (silica gel, EtOAc:hexanes $=3: 7)$ to give $\mathbf{6 a}(170 \mathrm{mg}, 70 \%)$ as an orange crystalline solid, $\mathrm{mp}$ 169.8-170.6 ${ }^{\circ} \mathrm{C} . \mathrm{R}_{f}$ (1:4 EtOAc/hexanes) 0.19; IR(thin film) 3321, 1688, 1629, 1442, 1311, 1182 $\mathrm{cm}^{-1} ;{ }^{1} \mathrm{H}$ NMR $\left(500 \mathrm{MHz}, \mathrm{CDCl}_{3}\right) \delta 1.28(\mathrm{~s}, 3 \mathrm{H}), 1.31(\mathrm{~s}, 6 \mathrm{H}), 2.22(\mathrm{~s}, 3 \mathrm{H}), 2.54(\mathrm{~s}, 2 \mathrm{H}), 7.25-$ $7.46(\mathrm{~m}, 5 \mathrm{H}), 9.70(\mathrm{~s}, 1 \mathrm{H}), 10.03(\mathrm{~s}, 1 \mathrm{H}), 11.05(\mathrm{br} \mathrm{s}, 1 \mathrm{H}) ;{ }^{13} \mathrm{C} \mathrm{NMR}\left(500 \mathrm{MHz}, \mathrm{CDCl}_{3}\right) \delta 8.9$, $10.2,25.8,46.4,46.8,123.7,128.5,129.2,129.3,130.2,130.4,131.4,134.2,138.8,153.1,177.6$, 178.9, 190.7. Anal. Calcd for $\mathrm{C}_{21} \mathrm{H}_{22} \mathrm{~N}_{2} \mathrm{O}_{2}$ : C, 75.42; H, 6.63; N, 8.38; O, 9.57. Found: C, 75.21; $\mathrm{H}, 6.70 ; \mathrm{N}, 8.39 .{ }^{1}$

\section{5-(5-Formyl-4,4-dimethyl-3,4-dihydro-pyrrol-2-ylidenemethyl)-3,4-dimethyl-1 $\mathrm{H}$-pyrrole-2-}

carbaldehyde (6c). Following the procedure outlined for $\mathbf{6 a}$, reaction of 13c $(166 \mathrm{mg}, 0.643$ $\mathrm{mmol})$ in $\mathrm{CH}_{2} \mathrm{Cl}_{2}(13 \mathrm{~mL})$ with pyridine $(66 \mu \mathrm{L}, 0.82 \mathrm{mmol})$ and $\mathrm{SeO}_{2}(91 \mathrm{mg}, 0.82 \mathrm{mmol})$ gave 6c $(107 \mathrm{mg}, 61 \%)$ after flash chromatography (silica gel, EtOAc:hexanes $=2: 3)$. Yellow crystalline solid, $\mathrm{mp} 115-117^{\circ} \mathrm{C} . \mathrm{R}_{f}(2: 3 \mathrm{EtOAc} /$ hexanes) 0.36 ; IR(thin film) $3374,1690,1635$ $\mathrm{cm}^{-1} ;{ }^{1} \mathrm{H}$ NMR $\left(500 \mathrm{MHz}, \mathrm{CDCl}_{3}\right) \delta 1.35(\mathrm{~s}, 6 \mathrm{H}), 2.05(\mathrm{~s}, 3 \mathrm{H}), 2.26(\mathrm{~s}, 3 \mathrm{H}), 2.74(\mathrm{~s}, 2 \mathrm{H}), 6.26(\mathrm{~s}$, $1 \mathrm{H}), 9.64(\mathrm{~s}, 1 \mathrm{H}), 9.99(\mathrm{~s}, 1 \mathrm{H}), 10.78($ br s, $1 \mathrm{H}) ;{ }^{13} \mathrm{C} \mathrm{NMR}\left(500 \mathrm{MHz}, \mathrm{CDCl}_{3}\right)$ \& 8.7, 8.8, 25.7, 46.4, 46.7, 112.3, 117.1, 122.6, 130.9, 133.8, 151.7, 177.2, 179.2, 190.5. HRMS (EI) Calcd for $\mathrm{C}_{15} \mathrm{H}_{18} \mathrm{~N}_{2} \mathrm{O}_{2}$ : 258.1368; found: 258.1361. Anal. Calcd for $\mathrm{C}_{15} \mathrm{H}_{18} \mathrm{~N}_{2} \mathrm{O}_{2}$ : C, 69.74; H, 7.02; N, 10.84; O, 12.39. Found: C, 69.61; H, 6.96; N, 10.68. ${ }^{1}$

5-[1-(5-Formyl-4,4-dimethyl-3,4-dihydro-pyrrol-2-ylidene)-hexyl]-3,4-dimethyl-1H-pyrrole2-carbaldehyde (6d). Following the procedure outlined for $\mathbf{6 a}$, reaction of 13d (144 $\mathrm{mg}, 0.458$ mmol) in $\mathrm{CH}_{2} \mathrm{Cl}_{2}(8.7 \mathrm{~mL})$ with pyridine $(44 \mu \mathrm{L}, 0.55 \mathrm{mmol})$ and $\mathrm{SeO}_{2}(61 \mathrm{mg}, 0.55 \mathrm{mmol})$ gave 6d $(94.7 \mathrm{mg}, 63 \%)$ as a yellow oil after flash chromatography (silica gel, EtOAc:hexanes $=2: 3$ ). 
$\mathrm{R}_{f}$ (2:3 EtOAc/hexanes) 0.50; IR(thin film) 3277, 1688, $1635 \mathrm{~cm}^{-1} ;{ }^{1} \mathrm{H}$ NMR (500 MHz, $\left.\mathrm{CDCl}_{3}\right)$ $\delta 0.90(\mathrm{t}, J=7.1,3 \mathrm{H}), 1.35(\mathrm{~m}, 4 \mathrm{H}), 1.37(\mathrm{~s}, 6 \mathrm{H}), 1.46(\mathrm{~m}, 2 \mathrm{H}), 2.13(\mathrm{~s}, 3 \mathrm{H}), 2.29(\mathrm{~s}, 3 \mathrm{H}), 2.53(\mathrm{~m}$, 2H), $2.74(\mathrm{~s}, 2 \mathrm{H}), 9.65(\mathrm{~s}, 1 \mathrm{H}), 9.91(\mathrm{~s}, 1 \mathrm{H}), 11.00$ (br s, $1 \mathrm{H}) ;{ }^{13} \mathrm{C} \mathrm{NMR}\left(500 \mathrm{MHz}, \mathrm{CDCl}_{3}\right) \delta 8.9$, $10.9,14.2,22.6,25.9,28.7,31.9,32.1,45.5,46.1,121.4,129.9,130.7,131.3,134.7,152.3,177.2$, 177.5, 190.5. HRMS (EI) Calcd for $\mathrm{C}_{20} \mathrm{H}_{28} \mathrm{~N}_{2} \mathrm{O}_{2}$ : 328.2151; found: 328.2143 .

\section{5-[1-(5-Formyl-4,4-dimethyl-3,4-dihydro-pyrrol-2-ylidene)-undecyl]-3,4-dimethyl-1H-}

pyrrole-2-carbaldehyde (6e). Following the procedure outlined for $\mathbf{6 a}$, reaction of $13 \mathbf{e}(131 \mathrm{mg}$, $0.341 \mathrm{mmol})$ in $\mathrm{CH}_{2} \mathrm{Cl}_{2}(6.5 \mathrm{~mL})$ with pyridine $(33 \mu \mathrm{L}, 0.41 \mathrm{mmol})$ and $\mathrm{SeO}_{2}(45 \mathrm{mg}, 0.41 \mathrm{mmol})$ gave 6e $(88.2 \mathrm{mg}, 65 \%)$ as a yellow oil after flash chromatography (silica gel, EtOAc:hexanes $=$ 1:4). $\mathrm{R}_{f}$ (1:4 EtOAc/hexanes) 0.33; IR(thin film) 3268, 1689, $1630 \mathrm{~cm}^{-1} ;{ }^{1} \mathrm{H}$ NMR (500 MHz, $\left.\mathrm{CDCl}_{3}\right) \delta 0.89(\mathrm{t}, J=6.8,3 \mathrm{H}), 1.34-1.22(\mathrm{~m}, 14 \mathrm{H}), 1.38(\mathrm{~s}, 6 \mathrm{H}), 1.51-1.42(\mathrm{~m}, 2 \mathrm{H}), 2.14(\mathrm{~s}, 3 \mathrm{H})$, $2.30(\mathrm{~s}, 3 \mathrm{H}), 2.52-2.57(\mathrm{~m}, 2 \mathrm{H}), 2.75(\mathrm{~s}, 2 \mathrm{H}), 9.66(\mathrm{~s}, 1 \mathrm{H}), 9.92(\mathrm{~s}, 1 \mathrm{H}), 11.00(\mathrm{br} \mathrm{s}, 1 \mathrm{H}) ;{ }^{13} \mathrm{C}$ NMR (500 MHz, $\left.\mathrm{CDCl}_{3}\right) \delta$ 9.0, 11.0, 14.3, 22.9, 26.0, 29.2, 29.5, 29.66, 29.75, 29.79, 29.9, 32.1, 32.2, 45.6, 46.1, 121.5, 130.0, 130.8, 131.4, 134.6, 152.3, 177.4, 177.7, 190.6. HRMS (EI) Calcd for $\mathrm{C}_{25} \mathrm{H}_{38} \mathrm{~N}_{2} \mathrm{O}_{2}$ : 398.2933; found: 398.2932 .

\section{Preparation of Other A,B-ring intermediates:}

\section{3,4-Dimethyl-2-[phenyl-(4,4,5-trimethyl-3,4-dihydro-pyrrol-2-ylidene)-methyl]-1H-pyrrole}

(17a). $33 \% \mathrm{HBr}$ in HOAc $(450 \mu \mathrm{L}, 2.62 \mathrm{mmol})$ was added to $\mathbf{1 5 a}(103 \mathrm{mg}, 0.262 \mathrm{mmol})$, and the solution was stirred at $\mathrm{rt}$ for $30 \mathrm{~min}$. The mixture was then added to $10 \% \mathrm{KH}_{2} \mathrm{PO}_{4}(5 \mathrm{~mL})$ at $0^{\circ} \mathrm{C}$ and extracted with $\mathrm{CH}_{2} \mathrm{Cl}_{2}(3 \times 5 \mathrm{~mL})$. The combined organic extracts were washed sequentially with saturated aq $\mathrm{NaHCO}_{3}$ and brine, dried over $\mathrm{Na}_{2} \mathrm{SO}_{4}$, filtered, and concentrated. The resulting oil, a mixture of Z-17a (major) and E-17a (minor) isomers, was NMR-clean and generally used immediately due to the instability of the product. For analytical purposes, Z-17a (56.7 mg, 74\%) was isolated as a yellow film by flash chromatography ( unstable to prolonged handling and mass analysis. $\mathrm{R}_{f}$ (1:4 EtOAc/hexanes) 0.60 ; IR(thin film) 3316, 1701, $1441 \mathrm{~cm}^{-1}$; ${ }^{1} \mathrm{H}$ NMR $\left(500 \mathrm{MHz}, \mathrm{CDCl}_{3}\right) \delta 1.13(\mathrm{~s}, 6 \mathrm{H}), 1.25(\mathrm{~s}, 3 \mathrm{H}), 1.97(\mathrm{~s}, 3 \mathrm{H})$, $2.16(\mathrm{~s}, 3 \mathrm{H}), 2.34(\mathrm{~s}, 2 \mathrm{H}), 6.65(\mathrm{~s}, 1 \mathrm{H}), 7.29-7.41(\mathrm{~m}, 5 \mathrm{H}), 1.08($ br s, $1 \mathrm{H}) ;{ }^{13} \mathrm{C}$ NMR $(500 \mathrm{MHz}$, $\left.\mathrm{CDCl}_{3}\right) \delta 10.3,10.7,15.9,25.9,44.5,47.9,115.9,117.8,119.2,122.1,126.9,128.0,128.6,130.1$, $141.1,146.6,185.1$.

\section{5-(5-[1,3]Dithian-2-yl-4,4-dimethyl-2,5-dioxo-pentyl)-3,4-dimethyl-1Hpyrrole-2-carb-}

aldehyde (24cB). A solution of 1,3 dithiane $(214 \mathrm{mg}, 1.78 \mathrm{mmol})$ in THF $(9.0 \mathrm{~mL})$ was cooled to $-40^{\circ} \mathrm{C}$ and treated dropwise with $2.5 \mathrm{M} \mathrm{nBuLi}$ in hexanes $(0.81 \mathrm{~mL}, 1.7 \mathrm{mmol})$ over $5 \mathrm{~min}$. The solution was stirred at $-40^{\circ} \mathrm{C}$ for $5 \mathrm{~min}$, then at $-15^{\circ} \mathrm{C}$ for an additional $2 \mathrm{~h}$. The lithium dithiane reagent was then added dropwise to a solution of $22 \mathrm{c}(200 \mathrm{mg}, 0.81 \mathrm{mmol})$ in THF $(10 \mathrm{~mL})$ at $-78^{\circ} \mathrm{C}$. After addition was complete, the reaction mixture was stirred at $-78^{\circ} \mathrm{C}$ for $30 \mathrm{~min}$. It was then quenched by addition to saturated aq $\mathrm{NH}_{4} \mathrm{Cl}(20 \mathrm{~mL})$. After the mixture had warmed to rt, $\mathrm{Et}_{2} \mathrm{O}(20 \mathrm{~mL})$ was added, and the layers were separated. The organic layer was washed sequentially with water and brine, dried over $\mathrm{Na}_{2} \mathrm{SO}_{4}$, filtered and concentrated. The residue was purified by flash chromatography (silica gel, EtOAc:hexanes $=2: 5)$ to give $\mathbf{2 4 c B}(220 \mathrm{mg}, 74 \%)$ as colorless crystalline solid, $\mathrm{mp}$ 142.3-142.9 ${ }^{\circ} \mathrm{C} . \mathrm{R}_{f}$ (2:5 EtOAc/hexanes) 0.15 ; IR(thin film) 3246, 2919, 1695, $1627 \mathrm{~cm}^{-1} ;{ }^{1} \mathrm{H}$ NMR $\left(500 \mathrm{MHz}, \mathrm{CDCl}_{3}\right) \delta 1.31(\mathrm{~s}, 1 \mathrm{H}), 1.91(\mathrm{~s}, 1 \mathrm{H}), 2.00(\mathrm{~m}$, 
$1 \mathrm{H}), 2.12(\mathrm{~m}, 1 \mathrm{H}), 2.26(\mathrm{~s}, 3 \mathrm{H}), 2.57(\mathrm{~m}, 2 \mathrm{H}), 2.83(\mathrm{~s}, 2 \mathrm{H}), 3.27$ (ddd, $J=13.67,11.96,2.68,2 \mathrm{H})$, $3.71(\mathrm{~s}, 2 \mathrm{H}), 4.69(\mathrm{~s}, 1 \mathrm{H}), 9.50(\mathrm{~s}, 1 \mathrm{H}), 10.11(\mathrm{br} \mathrm{s}, 1 \mathrm{H}) ;{ }^{13} \mathrm{C} \mathrm{NMR}\left(500 \mathrm{MHz}, \mathrm{CDCl}_{3}\right) \delta 8.7,9.1$, 25.3, 26.0, 26.1, 40.4, 41.7, 45.6, 54.4, 119.6, 128.9, 131.5, 132.5, 176.7, 204.8, 207.6. Anal. Calcd for $\mathrm{C}_{18} \mathrm{H}_{25} \mathrm{NO}_{3} \mathrm{~S}_{2}$ : C, 58.82; H, 6.86; N, 3.81; O, 13.06; S, 17.45. Found: C, 58.93; H, 6.92; $\mathrm{N}, 3.90 ; \mathrm{S}, 17.28$.

5-[1-(4,4-Dimethyl-3-oxo-2-phenylsulfanyl-cyclopent-1-enyl)-ethyl]-3,4-dimethyl-1H-pyrrole2-carbaldehyde (25). A solution of thioanisole $(100 \mu \mathrm{L}, 0.855 \mathrm{mmol})$ and TMEDA $(120 \mu \mathrm{L}$, $0.817 \mathrm{mmol})$ in THF $(2.1 \mathrm{~mL})$ was cooled to $0^{\circ} \mathrm{C}$ and treated dropwise with $2.5 \mathrm{M} \mathrm{nBuLi}$ in hexanes $(0.34 \mathrm{~mL}, 0.82 \mathrm{mmol})$ over $10 \mathrm{~min}$. The solution was stirred at $0^{\circ} \mathrm{C}$ for $15 \mathrm{~min}$, then at $\mathrm{rt}$ for an additional $2.5 \mathrm{~h}$. The lithiothioanisole reagent was then added dropwise to a solution of $\mathbf{2 2 b}$ (102 $\mathrm{mg}, 0.389 \mathrm{mmol})$ in THF $(1.5 \mathrm{~mL})$ at $-78^{\circ} \mathrm{C}$. The reaction was quenched by addition to saturated aq $\mathrm{NH}_{4} \mathrm{Cl}(20 \mathrm{~mL})$. After the mixture had warmed to $\mathrm{rt}, \mathrm{Et}_{2} \mathrm{O}(20 \mathrm{~mL})$ was added, and the layers were separated. The organic layer was washed sequentially with water and brine, dried over $\mathrm{Na}_{2} \mathrm{SO}_{4}$, filtered and concentrated. The residue was purified by flash chromatography (silica gel, EtOAc:hexanes $=3: 7)$ to give $\mathbf{2 4 b C}(86.5 \mathrm{mg}, 78 \%)$ that was unstable and readily underwent aldol reaction to give $\mathbf{2 5}$ upon handling. Complete conversion of $\mathbf{2 4 b C}$ to $\mathbf{2 5}$ was accomplished following the procedure outlined for 13a: Reaction of $24 \mathbf{b C}(50.1 \mathrm{mg}, 0.130 \mathrm{mmol})$ in $\mathrm{CH}_{3} \mathrm{CN}$ $(1.4 \mathrm{~mL})$ with $\left(\mathrm{NH}_{4}\right)_{2} \mathrm{CO}_{3}(130 \mathrm{mg}, 1.30 \mathrm{mmol})$ and basic alumina $(250 \mathrm{mg}$, Grade I) gave 25 (39.6 mg, 83\%) after flash chromatography (silica gel, EtOAc:hexanes = 3:7). Colorless crystalline solid, $\mathrm{mp} 187.2-188.2^{\circ} \mathrm{C} . \mathrm{R}_{f}(2: 3$ EtOAc/hexanes) 0.22 ; IR(thin film) 1709, 1629, 1439, $1138 \mathrm{~cm}^{-1} ;{ }^{1} \mathrm{H}$ NMR $\left(500 \mathrm{MHz}, \mathrm{CDCl}_{3}\right) \delta 1.06(\mathrm{~s}, 3 \mathrm{H}), 1.14(\mathrm{~s}, 3 \mathrm{H}), 1.49(\mathrm{~d}, J=7.3,3 \mathrm{H}), 1.82(\mathrm{~s}$, $3 \mathrm{H}), 2.14(\mathrm{~d}, J=18.6,1 \mathrm{H}), 2.24(\mathrm{~s}, 3 \mathrm{H}), 2.54(\mathrm{~d}, J=18.6,1 \mathrm{H}), 4.78(\mathrm{q}, J=7.3,1 \mathrm{H}), 7.18-7.27$ (m, 5H), 9.05 (br s, 1H), $\left.9.55(\mathrm{~s}, 1 \mathrm{H}) ;{ }^{13} \mathrm{C} \mathrm{NMR} \mathrm{(500} \mathrm{MHz,} \mathrm{CDCl}_{3}\right) \delta 8.7,9.0,16.4,25.3,25.5$, 33.7, 43.5, 43.8, 119.3, 126.8, 128.8, 128.9, 129.3, 130.6, 132.5, 134.4, 136.8, 176.9, 179.5, 209.2. Anal. Calcd for $\mathrm{C}_{22} \mathrm{H}_{25} \mathrm{NO}_{2} \mathrm{~S}: \mathrm{C}, 71.90 ; \mathrm{H}, 6.86 ; \mathrm{N}, 3.81 ; \mathrm{O}, 8.71 ; \mathrm{S}, 8.73$. Found: $\mathrm{C}, 72.02 ; \mathrm{H}$, $7.03 ; \mathrm{N}, 3.92 ; \mathrm{S}, 8.64$.

\section{Preparation of C,D-rings:}
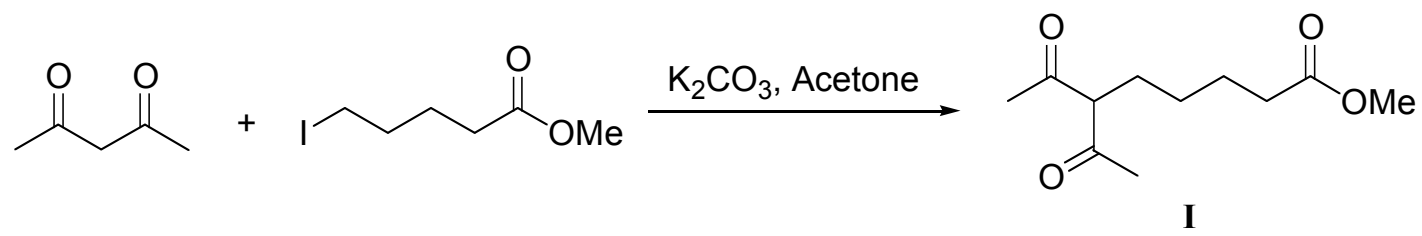

6-Acetyl-7-oxo-octanoic acid methyl ester (I). A solution of methyl 5-iodovalerate (2.32 g, 9.58 mmol), 2, 4-pentandione (0.960 g, $9.58 \mathrm{mmol}), \mathrm{K}_{2} \mathrm{CO}_{3}(1.33 \mathrm{~g}, 9.58 \mathrm{mmol})$ in acetone $(30 \mathrm{~mL})$ was refluxed for $24 \mathrm{~h}$. The solvent was removed by rotary evaporation. The residue was then dissolved in $\mathrm{CH}_{2} \mathrm{Cl}_{2}(50 \mathrm{~mL})$, washed sequentially with water $(20 \mathrm{~mL} \times 2)$ and brine $(20 \mathrm{~mL})$, dried over $\mathrm{Na}_{2} \mathrm{SO}_{4}$, and concentrated to give crude $\mathbf{I}$ (1.92 g, $94 \%$ ), which was used directly for the next step. ${ }^{2}$ 


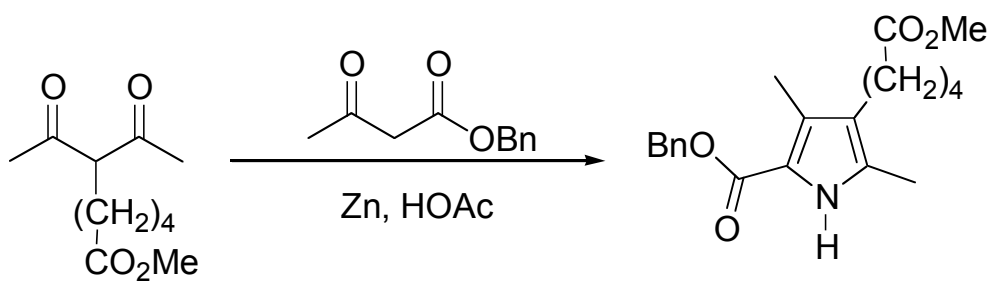

II

4-Methoxycarbonylmethyl-3,5-dimethyl-1H-pyrrole-2-carboxylic acid benzyl ester (II). A solution of sodium nitrite $(725 \mathrm{mg}, 10.5 \mathrm{mmol})$ in water $(5 \mathrm{~mL})$ was added dropwise to a wellstirred solution of benzyl acetoacetate $(1.44 \mathrm{~g}, 7.51 \mathrm{mmol})$ in $\mathrm{AcOH}(5 \mathrm{~mL})$ at $0{ }^{\circ} \mathrm{C}$. The resulting mixture was stirred at $\mathrm{rt}$ for $6 \mathrm{~h}$. The solution was then extracted with $\mathrm{Et}_{2} \mathrm{O}(3 \times 20 \mathrm{~mL})$. The combined organic extracts were washed with brine, dried over $\mathrm{Na}_{2} \mathrm{SO}_{4}$, and concentrated. The resulting oil was added dropwise, along with zinc metal $(1.473 \mathrm{~g}, 22.53 \mathrm{mmol})$, to a solution of methyl 6-acetyl-7-oxooctanoate $(1.609 \mathrm{~g}, 7.51 \mathrm{mmol})$ in $\mathrm{AcOH}(3 \mathrm{~mL})$ while maintaining a reaction temperature below $65{ }^{\circ} \mathrm{C}$. After addition was complete, the reaction mixture was stirred at $\mathrm{rt}$ for $6 \mathrm{~h}$, then poured into ice-water and extracted with EtOAc $(3 \times 40 \mathrm{~mL})$. The combined organic extracts were washed sequentially with water $(2 \times 50 \mathrm{~mL})$ and brine, dried over $\mathrm{Na}_{2} \mathrm{SO}_{4}$, and concentrated. A crude purification was performed on the resulting oil (silica gel, hexanes:EtOAc $=4: 1)$ to give II $(1.3 \mathrm{~g}, 50 \%, \sim 90 \%$ purity), which was used without further purification for the subsequent step. ${ }^{2}$
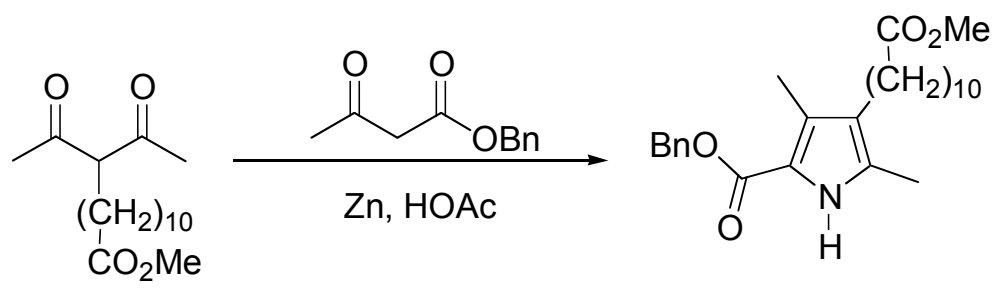

III

4-Methoxycarbonylmethyl-3,5-dimethyl-1H-pyrrole-2-carboxylic acid benzyl ester (III). Following the procedure outlined for II, reaction of sodium nitrite $(693 \mathrm{mg}, 10.0 \mathrm{mmol})$ in water $(5 \mathrm{~mL})$, benzyl acetoacetate $(1.38 \mathrm{~g}, 7.17 \mathrm{mmol})$ in AcOH $(5 \mathrm{~mL})$ with zinc metal $(1.407 \mathrm{~g}, 21.52$ mmol) and methyl 12-acetyl-13-oxotetradecanoate $(2.14 \mathrm{~g}, 7.17 \mathrm{mmol})$ in AcOH $(3 \mathrm{~mL})$ gave III $(1.46 \mathrm{~g}, 48 \%)$ after flash chromatography (silica gel, hexanes:EtOAc $=5: 1)$. Solid, 66.5-68.0 ${ }^{\circ} \mathrm{C}$. IR(thin film) 3313, 1738, 1664, 1438, $1267 \mathrm{~cm}^{-1} ;{ }^{1} \mathrm{H}$ NMR $\left(500 \mathrm{MHz}, \mathrm{CDCl}_{3}\right) \delta 1.19(\mathrm{~m}, 12 \mathrm{H})$, $1.31(\mathrm{~m}, 2 \mathrm{H}), 1.53(\mathrm{~m}, 2 \mathrm{H}), 2.09(\mathrm{~s}, 3 \mathrm{H}), 2.20(\mathrm{~s}, 3 \mathrm{H}), 2.25(\mathrm{~m}, 4 \mathrm{H}), 3.58(\mathrm{~s}, 3 \mathrm{H}), 5.21(\mathrm{~s}, 2 \mathrm{H})$, $7.32(\mathrm{~m}, 5 \mathrm{H}), 8.61(\mathrm{~s}, 1 \mathrm{H}) ;{ }^{13} \mathrm{C}$ NMR $\left(500 \mathrm{MHz}, \mathrm{CDCl}_{3}\right) \delta 11.0,11.8,24.3,25.2,29.4,29.5,29.7$, 29.8, 29.8, 31.1, 34.4, 51.7, 65.6, 116.5, 122.8, 127.9, 128.2, 128.3, 128.8, 130.2, 137.0, 161.6, 174.6. HRMS (ESI) Calcd for $\mathrm{C}_{26} \mathrm{H}_{37} \mathrm{NO}_{4}$ : 427.2723 ; found: $428.2783\left(\mathrm{M}+\mathrm{H}^{+}\right)$. 

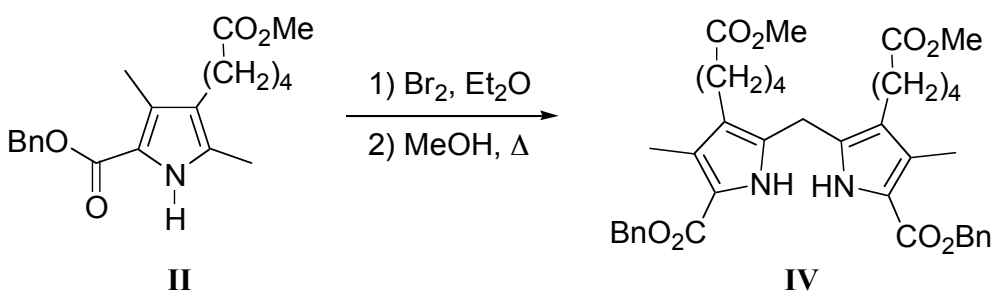

Dibenzyl 3,3'-Bis-(4-methoxycarbonylbutyl)-4,4'-dimethylpyrromethane-5,5'-dicarboxylate (IV). A solution of II $(0.969 \mathrm{~g}, 2.82 \mathrm{mmol})$ in $\mathrm{Et}_{2} \mathrm{O}(23 \mathrm{~mL})$ was treated dropwise with $\mathrm{Br}_{2}$ (519 $\mathrm{mg}, 3.25 \mathrm{mmol})$ in $\mathrm{Et}_{2} \mathrm{O}(9.8 \mathrm{~mL})$ over $5 \mathrm{~min}$, then stirred at $\mathrm{rt}$ for $2 \mathrm{~h}$. The solvent was removed by rotary evaporation, and the residual pink solid was taken up in $\mathrm{MeOH}(9 \mathrm{~mL})$ and heated at reflux for $3 \mathrm{~h}$. The solution was allowed to cool to rt, concentrated, and the residue was purified by flash chromatography (silica gel, hexanes:EtOAc $=4: 1)$ to give IV $(0.678 \mathrm{~g}, 72 \%)$ as a solid, $\mathrm{mp} 85.0-86.0^{\circ} \mathrm{C}$. IR(thin film) $3311,1735,1687,1438,1250 \mathrm{~cm}^{-1} ;{ }^{1} \mathrm{H}$ NMR $\left(500 \mathrm{MHz}, \mathrm{CDCl}_{3}\right)$ ठ $1.40(\mathrm{~m}, 4 \mathrm{H}), 1.63(\mathrm{~m}, 4 \mathrm{H}), 2.25(\mathrm{~s}, 6 \mathrm{H}), 2.28(\mathrm{~m}, 4 \mathrm{H}), 2.35(\mathrm{t}, J=7.5 \mathrm{~Hz}, 4 \mathrm{H}), 3.65(\mathrm{~s}, 6 \mathrm{H}), 3.83$ (s, 2H), $5.26(\mathrm{~s}, 4 \mathrm{H}), 7.36(\mathrm{~m}, 10 \mathrm{H}), 8.77(\mathrm{~s}, 2 \mathrm{H}) ;{ }^{13} \mathrm{C} \mathrm{NMR}\left(500 \mathrm{MHz}, \mathrm{CDCl}_{3}\right) \delta$ 11.0, 23.5, 23.9, 25.0, 30.5, 34.1, 51.8, 65.8, 117.8, 122.5, 127.9, 128.2, 128.2, 128.7, 129.9, 136.6, 161.8, 174.3. Anal. Calcd for $\mathrm{C}_{39} \mathrm{H}_{46} \mathrm{~N}_{2} \mathrm{O}_{8}: \mathrm{C}, 69.83 ; \mathrm{H}, 6.91 ; \mathrm{N}, 4.18$. Found: C, 69.41; H, 6.98; N, 4.07. ${ }^{3}$

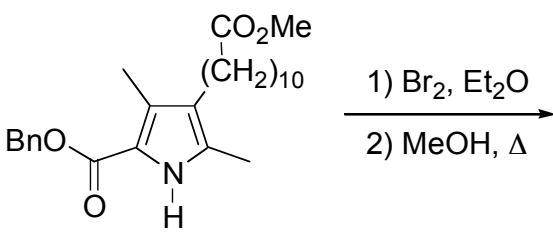

III

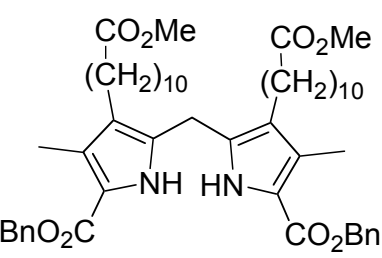

V

Dibenzyl 3,3'-Bis-(10-methoxycarbonyldecyl)-4,4'-dimethylpyrromethane-5,5'-dicarboxylate (V). Following the procedure outlined for IV, reaction of III $(2.03 \mathrm{~g}, 4.75 \mathrm{mmol})$ in $\mathrm{Et}_{2} \mathrm{O}(48 \mathrm{~mL})$ with $\mathrm{Br}_{2}(874 \mathrm{mg}, 6.47 \mathrm{mmol})$ in $\mathrm{Et}_{2} \mathrm{O}(15.6 \mathrm{~mL})$ and $\mathrm{MeOH}(18 \mathrm{~mL})$ gave $\mathbf{V}(1.12 \mathrm{~g}, 56 \%)$ after flash chromatography (silica gel, hexanes:EtOAc $=4: 1$ ). Colorless crystalline solid, $64.0-65 \cdot 0^{\circ} \mathrm{C}$. IR(thin film) 3312, 1738, 1701, 1656, 1438, $1252 \mathrm{~cm}^{-1} ;{ }^{1} \mathrm{H}$ NMR (500 MHz, $\left.\mathrm{CDCl}_{3}\right) \delta 1.21(\mathrm{~m}$, $24 \mathrm{H}), 1.28(\mathrm{~m}, 4 \mathrm{H}), 1.55(\mathrm{~m}, 4 \mathrm{H}), 2.21(\mathrm{~s}, 6 \mathrm{H}), 2.23(\mathrm{~m}, 4 \mathrm{H}), 2.27(\mathrm{~m}, 4 \mathrm{H}), 3.60(\mathrm{~s}, 6 \mathrm{H}), 3.74(\mathrm{~s}$, $2 \mathrm{H}), 5.17(\mathrm{~s}, 4 \mathrm{H}), 7.26(\mathrm{~m}, 10 \mathrm{H}), 8.88(\mathrm{~s}, 2 \mathrm{H}) ;{ }^{13} \mathrm{C}$ NMR $\left(500 \mathrm{MHz}, \mathrm{CDCl}_{3}\right) \delta 11.0,23.0,24.3$, 25.0, 29.3, 29.4, 29.6, 29.7, 29.7, 29.8, 31.2, 34.2, 51.5, 65.7, 117.5, 122.7, 127.8, 127.8, 128.0, 128.5, 130.6, 136.4, 162.0, 174.4. Anal. Calcd for $\mathrm{C}_{51} \mathrm{H}_{70} \mathrm{~N}_{2} \mathrm{O}_{8}: \mathrm{C}, 73.00 ; \mathrm{H}, 8.41 ; \mathrm{N}, 3.34$. Found: C, 73.02; H, 8.52; N, 3.31. ${ }^{3}$ 

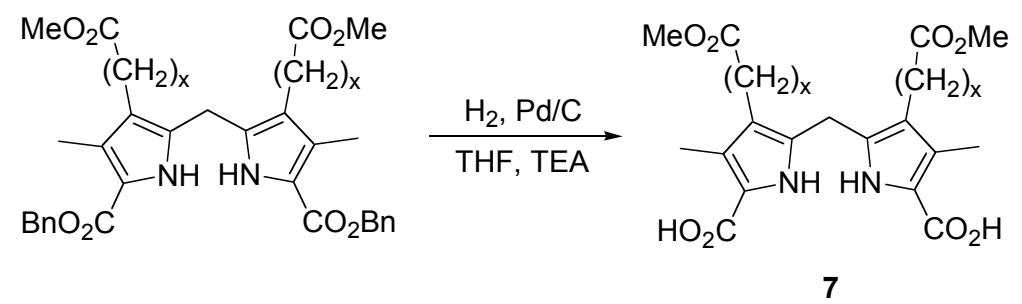

3,3'-Bis-(4-methoxycarbonylbutyl)-4,4'-dimethylpyrromethane-5,5'-dicarboxylic acid (7d). A solution of IV $(201 \mathrm{mg}, 0.300 \mathrm{mmol})$ in THF $(3.6 \mathrm{~mL})$ and $\mathrm{NEt}_{3}(7.2 \mu \mathrm{L})$ was treated with $\mathrm{Pd} / \mathrm{C}$ (19.3 $\mathrm{mg}, 0.018 \mathrm{mmol}$ ) and stirred at $\mathrm{rt}$ under an $\mathrm{H}_{2}$ atmosphere in the dark for $6 \mathrm{~h}$. The solution was filtered through Celite and concentrated to give $7 \mathbf{d}(0.147 \mathrm{~g}$, 99\%), which could be crystallized from $\mathrm{MeOH}$ to give a colorless crystalline solid, mp 130-132 ${ }^{\circ} \mathrm{C}$. IR(thin film) 3309 , 1737, 1653, 1465, $1283 \mathrm{~cm}^{-1}$; ${ }^{1} \mathrm{H}$ NMR $\left(500 \mathrm{MHz}, \mathrm{CDCl}_{3}\right) \delta 1.13(\mathrm{~m}, 4 \mathrm{H}), 1.50(\mathrm{~m}, 4 \mathrm{H}), 2.11(\mathrm{~s}$, $6 \mathrm{H}), 2.24(\mathrm{~m}, 8 \mathrm{H}), 3.55(\mathrm{~s}, 6 \mathrm{H}), 3.72(\mathrm{~s}, 2 \mathrm{H}), 11.07(\mathrm{~s}, 2 \mathrm{H}), 11.99(\mathrm{~s}, 2 \mathrm{H}) ;{ }^{13} \mathrm{C}$ NMR $(500 \mathrm{MHz}$, $\left.\mathrm{CDCl}_{3}\right) \delta 11.0,24.0,25.2,30.6,33.9,51.8,51.9,117.7,121.2,125.8,131.2,163.2,174.0$. HRMS (ESI) Calcd for $\mathrm{C}_{25} \mathrm{H}_{34} \mathrm{~N}_{2} \mathrm{O}_{8}: 490.2315$; found: $447.2473\left(\mathrm{M}+\mathrm{H}^{+}\right.$; loss of $\left.\mathrm{CO}_{2}\right){ }^{3}$

3,3'-Bis-(10-methoxycarbonyldecyl)-4,4'-dimethylpyrromethane-5,5'-dicarboxylic acid (7e). Following the procedure outlined for $\mathbf{7 d}$, reaction of $\mathbf{V}(252 \mathrm{mg}, 0.300 \mathrm{mmol})$ in THF $(3.6 \mathrm{~mL})$ and $\mathrm{NEt}_{3}(7.2 \mu \mathrm{L})$ with $\mathrm{Pd} / \mathrm{C}(19.3 \mathrm{mg}, 0.018 \mathrm{mmol})$ gave $7 \mathrm{e}(0.198 \mathrm{~g}, 99 \%)$, which could be crystallized from $\mathrm{MeOH}$ to give a colorless crystalline solid, mp 118-120 ${ }^{\circ} \mathrm{C}$. IR(thin film) 3315 , 1739, 1651, 1465, $1258 \mathrm{~cm}^{-1} ;{ }^{1} \mathrm{H}$ NMR $\left(500 \mathrm{MHz}, \mathrm{CDCl}_{3}\right) \delta 1.13(\mathrm{~m}, 4 \mathrm{H}), 1.22(\mathrm{~m}, 24 \mathrm{H}), 1.50$ $(\mathrm{m}, 4 \mathrm{H}), 2.11(\mathrm{~s}, 6 \mathrm{H}), 2.27(\mathrm{~m}, 8 \mathrm{H}), 3.57(\mathrm{~s}, 6 \mathrm{H}), 3.71(\mathrm{~s}, 2 \mathrm{H}), 11.05(\mathrm{~s}, 2 \mathrm{H}), 11.94(\mathrm{~s}, 2 \mathrm{H}) ;{ }^{13} \mathrm{C}$ NMR $\left(500 \mathrm{MHz}, \mathrm{CDCl}_{3}\right) \delta 11.0,24.5,25.1,29.2,29.4,29.6,29.7,29.7,29.9,31.3,34.0,51.8$, 51.9, 117.7, 121.6, 125.7, 131.2, 163.2, 174.0. HRMS (ESI) Calcd for $\mathrm{C}_{37} \mathrm{H}_{58} \mathrm{~N}_{2} \mathrm{O}_{8}$ : 658.4193; found: $659.4259\left(\mathrm{M}+\mathrm{H}^{+}\right) .^{3}$

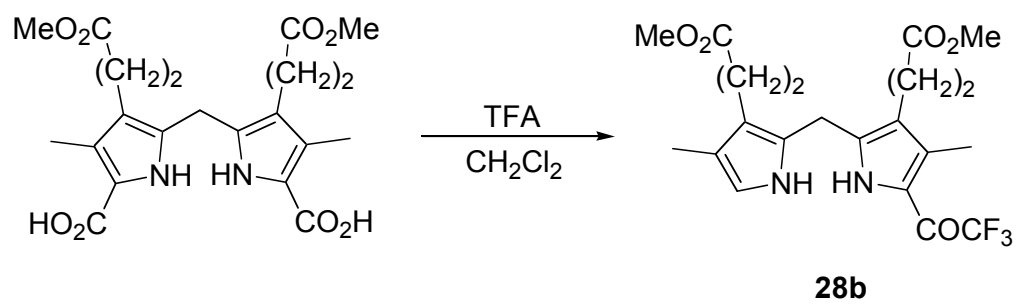

3-\{2-[3-(2-Methoxycarbonyl-ethyl)-4-methyl-5-trifluoroacetyl-1H-pyrrol-2-ylmethyl]-4methyl-1H-pyrrol-3-yl\}-propionic acid methyl ester (28b). A solution of $7 \mathbf{b}(20 \mathrm{mg}, 46 \mu \mathrm{mol})$ in $\mathrm{CH}_{2} \mathrm{Cl}_{2}(2 \mathrm{~mL})$ was treated with TFA $(200 \mu \mathrm{L}, 2.7 \mathrm{mmol})$ and stirred at $\mathrm{rt}$ for $2 \mathrm{~h}$. The solution was then added to $10 \%$ aq $\mathrm{KH}_{2} \mathrm{PO}_{4}(10 \mathrm{~mL})$ at $0^{\circ} \mathrm{C}$. The layers were separated, and the aqueous layer was extracted with $\mathrm{CH}_{2} \mathrm{Cl}_{2}(5 \mathrm{~mL})$. The combined organic extracts were washed sequentially with water and brine, dried over $\mathrm{Na}_{2} \mathrm{SO}_{4}$, filtered and concentrated. The residue was purified by flash chromatography (silica gel, EtOAc:hexanes $=1: 9)$ to give $\mathbf{2 8 b}(11 \mathrm{mg}, 55 \%)$ as a clear colorless oil. $\mathrm{R}_{f}\left(2: 3 \mathrm{EtOAc} /\right.$ hexanes) $0.40 ;{ }^{1} \mathrm{H}$ NMR $\left(500 \mathrm{MHz}, \mathrm{CDCl}_{3}\right) 2.06(\mathrm{~s}, 3 \mathrm{H}), 2.36$ (s, $3 \mathrm{H}), 2.59(\mathrm{~m}, 4 \mathrm{H}), 2.76(\mathrm{t}, J=6.8,2 \mathrm{H}), 2.84(\mathrm{t}, J=7.2,2 \mathrm{H}), 3.68(\mathrm{~s}, 3 \mathrm{H}), 3.70(\mathrm{~s}, 3 \mathrm{H}), 4.02(\mathrm{~s}, 2 \mathrm{H})$, 
6.45 (s, 1H), 8.27 (br s, $1 \mathrm{H}), 9.55$ (br s, $1 \mathrm{H}) ;{ }^{13} \mathrm{C} \mathrm{NMR}\left(500 \mathrm{MHz}, \mathrm{CDCl}_{3}\right) \delta$ 10.7, 11.5, 19.3, 19.5, 23.1, 34.4, 34.5, 52.0, 52.1, 115.5, 117.4 (q, $J=289$ ), 117.8, 117.9, 122.67, 122.75, 123.4, 123.8, 139.7, 168.3 (q, $J=35.9$ ), 174.0, 174.7; ${ }^{19} \mathrm{~F}$ NMR (300 MHz, $\mathrm{CDCl}_{3}$ ) $\delta$-73.1; HRMS (EI) Calcd for $\mathrm{C}_{21} \mathrm{H}_{25} \mathrm{~F}_{3} \mathrm{~N}_{2} \mathrm{O}_{5}$ : 442.1716; found: 442.1722. ${ }^{5}$

IV. Preparation of Chlorins 8:

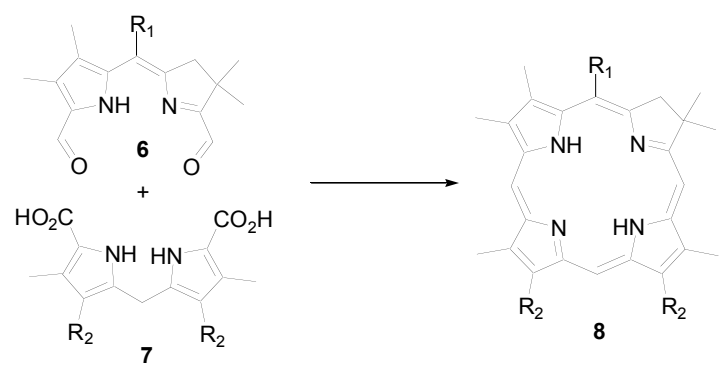

$\mathbf{2 , 2 , 7 , 8 , 1 2 , 1 3 , 1 7 , 1 8 - O c t a m e t h y l - 5 - p h e n y l - 2 , 3 , 2 2 , 2 4 - t e t r a h y d r o - p o r p h i n e ~ ( 8 a a ) . ~ N i t r o g e n ~ w a s ~}$ bubbled through a suspension of $\mathbf{6 a}(31 \mathrm{mg}, 0.093 \mathrm{mmol})$ and $7 \mathbf{a}(27 \mathrm{mg}, 0.093 \mathrm{mmol})$ in $\mathrm{CH}_{2} \mathrm{Cl}_{2}$ $(8.5 \mathrm{~mL})$ for $10 \mathrm{~min}$. The mixture was treated with TFA $(420 \mu \mathrm{L})$ and stirred at $\mathrm{rt}$ in the dark for $24 \mathrm{~h}$. The reaction was then poured into saturated aq $\mathrm{KHCO}_{3}(15 \mathrm{~mL})$ at $0^{\circ} \mathrm{C}$. The layers were separated, and the aqueous layer was extracted with $\mathrm{CH}_{2} \mathrm{Cl}_{2}(10 \mathrm{~mL})$. The combined organic layers were washed sequentially with water and brine, dried over $\mathrm{Na}_{2} \mathrm{SO}_{4}$, filtered, and concentrated. The residue was purified by chromatography (neutral alumina, Grade III, $\mathrm{CH}_{2} \mathrm{Cl}_{2}$ ) to give 8aa (34 mg, 74\%) as a green solid that was identical to the literature compound. ${ }^{1}$

3-[18-(2-Methoxycarbonyl-ethyl)-3,7,8,13,13,17-hexamethyl-10-phenyl-12,13,22,24-tetrahydro-porphin-2-yl]-propionic acid methyl ester (8ab). Following the procedure outlined for 8aa, reaction of $6 \mathbf{a}(43.2 \mathrm{mg}, 0.13 \mathrm{mmol})$ with $7 \mathbf{b}(56 \mathrm{mg}, 0.13 \mathrm{mmol})$ in $\mathrm{CH}_{2} \mathrm{Cl}_{2}(12 \mathrm{~mL})$ and TFA $(600 \mu \mathrm{L})$ gave 8ab (49 mg, 60\%) after chromatography (silica gel, EtOAc:hexanes =1:4, 1\% $\left.\mathrm{NEt}_{3}\right)$. The chlorin was identical to the literature compound. ${ }^{1}$

4-[18-(3-Methoxycarbonyl-propyl)-3,7,8,13,13,17-hexamethyl-10-phenyl-12,13,22,24-tetrahydro-porphin-2-yl]-butyric acid methyl ester (8ac). Following the procedure outlined for 8aa, reaction of $6 \mathbf{a}(28 \mathrm{mg}, 0.084 \mathrm{mmol})$ with $7 \mathbf{c}(39 \mathrm{mg}, 0.084 \mathrm{mmol})$ in $\mathrm{CH}_{2} \mathrm{Cl}_{2}(7.6 \mathrm{~mL})$ and TFA $(380 \mu \mathrm{L})$ gave 8ac $(42 \mathrm{mg}, 75 \%)$ after chromatography (silica gel, EtOAc:hexanes $=1: 5,1 \%$ $\left.\mathrm{NEt}_{3}\right)$. Green solid, decomposes above $290^{\circ} \mathrm{C}$. IR(thin film) $3445,1643 \mathrm{~cm}^{-1} ; \mathrm{UV}\left(\mathrm{CH}_{2} \mathrm{Cl}_{2}\right): \lambda_{\max }$ $\left(\varepsilon \mathrm{L} \mathrm{mol}^{-1} \mathrm{~cm}^{-1}\right)=648(50600) \mathrm{nm} ;{ }^{1} \mathrm{H}$ NMR $\left(500 \mathrm{MHz}, \mathrm{CDCl}_{3}\right) \delta-2.39(\mathrm{~s}, 1 \mathrm{H}),-2.03(\mathrm{~s}, 1 \mathrm{H}), 1.97$ $(\mathrm{s}, 6 \mathrm{H}), 2.42(\mathrm{~s}, 3 \mathrm{H}), 2.58(\mathrm{~m}, 4 \mathrm{H}), 2.71(\mathrm{~m}, 4 \mathrm{H}), 3.45(\mathrm{~s}, 3 \mathrm{H}), 3.46(\mathrm{~s}, 3 \mathrm{H}), 3.48(\mathrm{~s}, 3 \mathrm{H}), 3.73(\mathrm{~m}$, $6 \mathrm{H}), 3.93(\mathrm{t}, J=7.3 \mathrm{~Hz}, 2 \mathrm{H}), 4.07(\mathrm{t}, J=6.8 \mathrm{~Hz}, 2 \mathrm{H}), 4.12(\mathrm{~s}, 2 \mathrm{H}), 7.71(\mathrm{~m}, 3 \mathrm{H}), 7.85(\mathrm{~m}, 2 \mathrm{H}), 8.82$ $(\mathrm{s}, 1 \mathrm{H}), 9.75(\mathrm{~s}, 1 \mathrm{H}), 9.80(\mathrm{~s}, 1 \mathrm{H}) ;{ }^{13} \mathrm{C} \mathrm{NMR}\left(500 \mathrm{MHz}, \mathrm{CDCl}_{3}\right) \delta 11.6,12.0,12.0,14.0,14.0$, 25.6, 25.9, 27.9, 28.3, 30.0, 31.8, 33.9, 34.0, 46.0, 51.8, 51.8, 52.9, 91.3, 98.8, 99.4, 111.7, 127.8, $128.6,129.3,129.6,132.0,132.5,133.8,135.7,136.9,137.1,137.5,138.4,140.2,144.0,149.9$, 150.9, 162.3, 173.2, 174.4, 174.7. HRMS (ESI) Calcd for $\mathrm{C}_{42} \mathrm{H}_{48} \mathrm{~N}_{4} \mathrm{O}_{4}$ : 672.3676; found: 673.3748 $\left(\mathrm{M}+\mathrm{H}^{+}\right)$. 
5-[18-(4-Methoxycarbonyl-butyl)-3,7,8,13,13,17-hexamethyl-10-phenyl-12,13,22,24-tetrahydro-porphin-2-yl]-pentanoic acid methyl ester (8ad). Following the procedure outlined for 8aa, reaction of 6a $(28 \mathrm{mg}, 0.084 \mathrm{mmol})$ with $7 \mathbf{d}(41 \mathrm{mg}, 0.084 \mathrm{mmol})$ in $\mathrm{CH}_{2} \mathrm{Cl}_{2}(7.6 \mathrm{~mL})$ and TFA $(380 \mu \mathrm{L})$ gave $8 \mathbf{a d}(40 \mathrm{mg}, 68 \%)$ after chromatography (silica gel, EtOAc:hexanes $=1: 4,1 \%$ $\mathrm{NEt}_{3}$ ). Green solid, mp 194.0-196.0 ${ }^{\circ} \mathrm{C}$. IR(thin film) $3421,1735 \mathrm{~cm}^{-1} ; \mathrm{UV}\left(\mathrm{CH}_{2} \mathrm{Cl}_{2}\right): \lambda_{\max }(\varepsilon \mathrm{L}$ $\left.\mathrm{mol}^{-1} \mathrm{~cm}^{-1}\right)=648(51200) \mathrm{nm} ;{ }^{1} \mathrm{H}$ NMR $\left(500 \mathrm{MHz} \mathrm{CDCl}_{3}\right) \delta-2.38(\mathrm{~s}, 1 \mathrm{H}),-2.01(\mathrm{~s}, 1 \mathrm{H}), 1.98(\mathrm{~s}$, $6 \mathrm{H}), 2.07(\mathrm{~m}, 4 \mathrm{H}), 2.29(\mathrm{~m}, 4 \mathrm{H}), 2.44(\mathrm{~s}, 3 \mathrm{H}), 2.51(\mathrm{~m}, 4 \mathrm{H}), 3.46(\mathrm{~s}, 3 \mathrm{H}), 3.47(\mathrm{~s}, 3 \mathrm{H}), 3.49(\mathrm{~s}$, $3 \mathrm{H}), 3.68(\mathrm{~s}, 6 \mathrm{H}), 3.91(\mathrm{t}, J=7.5 \mathrm{~Hz}, 2 \mathrm{H}), 4.03(\mathrm{t}, J=7.5 \mathrm{~Hz}, 2 \mathrm{H}), 4.13(\mathrm{~s}, 2 \mathrm{H}), 7.72(\mathrm{~m}, 3 \mathrm{H}), 7.86$ $(\mathrm{m}, 2 \mathrm{H}), 8.83(\mathrm{~s}, 1 \mathrm{H}), 9.61(\mathrm{~s}, 1 \mathrm{H}), 9.81(\mathrm{~s}, 1 \mathrm{H}) ;{ }^{13} \mathrm{C} \mathrm{NMR}\left(500 \mathrm{MHz}, \mathrm{CDCl}_{3}\right) \delta 11.7,12.0,12.0$, $12.1,14.0,14.0,25.4,25.5,26.1,26.4,31.8,32.4,32.9,34.4,34.5,46.1,51.8,51.8,52.9,91.3$, $98.5,99.4,111.7,127.8,128.6,129.1,129.6,132.0,132.5,133.7,135.7,137.0,137.3,137.4$, 138.4, 140.8, 144.1, 150.0, 150.9, 162.2, 173.2, 174.3, 174.5. HRMS (ESI) Calcd for $\mathrm{C}_{44} \mathrm{H}_{52} \mathrm{~N}_{4} \mathrm{O}_{4}$ : 700.3989; found: $701.4070\left(\mathrm{M}+\mathrm{H}^{+}\right)$.

11-[18-(10-Methoxycarbonyl-decyl)-3,7,8,13,13,17-hexamethyl-10-phenyl-12,13,22,24-tetrahydro-porphin-2-yl]-undecanoic acid methyl ester (8ae). Following the procedure outlined for 8aa, reaction of 6a $(36 \mathrm{mg}, 0.11 \mathrm{mmol})$ with $7 \mathrm{e}(71 \mathrm{mg}, 0.11 \mathrm{mmol})$ in $\mathrm{CH}_{2} \mathrm{Cl}_{2}(10 \mathrm{~mL})$ and TFA $(500 \mu \mathrm{L})$ gave 8 ae $(51 \mathrm{mg}, 54 \%)$ after chromatography (silica gel, EtOAc:hexanes $=1: 4,1 \%$ $\mathrm{NEt}_{3}$ ). Green solid, mp 137.0-138.0 ${ }^{\circ} \mathrm{C}$. IR(thin film) 3422, $1641 \mathrm{~cm}^{-1} ; \mathrm{UV}\left(\mathrm{CH}_{2} \mathrm{Cl}_{2}\right): \lambda_{\max }(\varepsilon \mathrm{L}$ $\left.\mathrm{mol}^{-1} \mathrm{~cm}^{-1}\right)=648(51800) \mathrm{nm} ;{ }^{1} \mathrm{H} \operatorname{NMR}\left(500 \mathrm{MHz}, \mathrm{CDCl}_{3}\right) \delta-2.36(\mathrm{~s}, 1 \mathrm{H}),-2.01(\mathrm{~s}, 1 \mathrm{H}), 1.31$ (br, $16 \mathrm{H}), 1.52(\mathrm{br}, 4 \mathrm{H}), 1.63(\mathrm{~m}, 4 \mathrm{H}), 1.72(\mathrm{~m}, 4 \mathrm{H}), 1.99(\mathrm{~s}, 6 \mathrm{H}), 2.25(\mathrm{~m}, 4 \mathrm{H}), 2.30(\mathrm{~m}, 4 \mathrm{H}), 2.44(\mathrm{~s}$, $3 \mathrm{H}), 3.47(\mathrm{~s}, 3 \mathrm{H}), 3.48(\mathrm{~s}, 3 \mathrm{H}), 3.49(\mathrm{~s}, 3 \mathrm{H}), 3.68(\mathrm{~s}, 6 \mathrm{H}), 3.87(\mathrm{t}, J=7.5 \mathrm{~Hz}, 2 \mathrm{H}), 3.99$ (t, $J=7.5 \mathrm{~Hz}$, 2H), $4.14(\mathrm{~s}, 2 \mathrm{H}), 7.72(\mathrm{~m}, 3 \mathrm{H}), 7.87(\mathrm{~m}, 2 \mathrm{H}), 8.83(\mathrm{~s}, 1 \mathrm{H}), 9.66(\mathrm{~s}, 1 \mathrm{H}), 9.82(\mathrm{~s}, 1 \mathrm{H}) ;{ }^{13} \mathrm{C} \mathrm{NMR}$ $\left(500 \mathrm{MHz}, \mathrm{CDCl}_{3}\right) \delta 11.7,12.0,12.1,14.0,14.0,25.2,26.5,26.8,29.4,29.4,29.5,29.6,29.8$, $29.8,29.9,30.0,30.0,30.1,30.2,30.2,31.8,33.1,33.6,34.4,46.1,51.7,52.9,91.1,98.8,99.4$, $111.7,127.7,128.5,128.9,129.4,131.8,132.6,134.0,135.4,136.8,137.0,138.3,138.5,141.5$, 144.2, 150.0, 151.2, 161.9, 173.1, 174.6. HRMS (ESI) Calcd for $\mathrm{C}_{56} \mathrm{H}_{76} \mathrm{~N}_{4} \mathrm{O}_{4}$ : 868.5867; found: $869.5970\left(\mathrm{M}+\mathrm{H}^{+}\right)$.

2,2,5,7,8,12,13,17,18-Nonamethyl-2,3,22,24-tetrahydro-porphine (8ba). Following the procedure outlined for 8aa, reaction of $\mathbf{6 b}(33 \mathrm{mg}, 0.12 \mathrm{mmol})$ with $7 \mathbf{a}(36 \mathrm{mg}, 0.12 \mathrm{mmol})$ in $\mathrm{CH}_{2} \mathrm{Cl}_{2}(11 \mathrm{~mL})$ and TFA $(560 \mu \mathrm{L})$ gave $8 \mathbf{b a}(38 \mathrm{mg}, 72 \%)$ after chromatography (silica gel, EtOAc:hexanes $\left.=1: 4,1 \% \mathrm{NEt}_{3}\right)$. To quench the reaction, $3 \mathrm{M} \mathrm{NH} \mathrm{NH}_{4} \mathrm{OH}$ was substituted for saturated aq $\mathrm{KHCO}_{3}$. The chlorin was identical to the literature compound. ${ }^{1}$

4-[18-(3-Methoxycarbonyl-propyl)-3,7,8,10,13,13,17-heptamethyl-12,13,22,24-tetrahydroporphin-2-yl]-butyric acid methyl ester (8bc). Following the procedure outlined for 8aa, reaction of $\mathbf{6 b}(27 \mathrm{mg}, 0.10 \mathrm{mmol})$ with $7 \mathbf{c}(46 \mathrm{mg}, 0.10 \mathrm{mmol})$ in $\mathrm{CH}_{2} \mathrm{Cl}_{2}(9 \mathrm{~mL})$ and TFA (450 $\mu \mathrm{L}$ ) gave 8 bc (45 mg, 74\%) after chromatography (silica gel, EtOAc:hexanes $=1: 4,1 \% \mathrm{NEt}_{3}$ ). Green solid, mp 171.0-172.0 ${ }^{\circ} \mathrm{C}$. IR(thin film) $3424,1735 \mathrm{~cm}^{-1} ; \mathrm{UV}\left(\mathrm{CH}_{2} \mathrm{Cl}_{2}\right): \lambda_{\max }\left(\varepsilon \mathrm{L} \mathrm{mol}^{-1} \mathrm{~cm}^{-}\right.$ $\left.{ }^{1}\right)=648(40100) \mathrm{nm} ;{ }^{1} \mathrm{H} \mathrm{NMR}\left(500 \mathrm{MHz}, \mathrm{CDCl}_{3}\right) \delta-2.48(\mathrm{~s}, 1 \mathrm{H}),-2.01(\mathrm{~s}, 1 \mathrm{H}), 2.06(\mathrm{~s}, 6 \mathrm{H}), 2.59$ $(\mathrm{m}, 4 \mathrm{H}), 2.72(\mathrm{~m}, 4 \mathrm{H}), 3.44(\mathrm{~s}, 3 \mathrm{H}), 3.45(\mathrm{~s}, 3 \mathrm{H}), 3.46(\mathrm{~s}, 3 \mathrm{H}), 3.50(\mathrm{~s}, 3 \mathrm{H}), 3.74(\mathrm{~s}, 3 \mathrm{H}), 3.75(\mathrm{~s}$, $3 \mathrm{H}), 3.80(\mathrm{~s}, 3 \mathrm{H}), 3.94(\mathrm{t}, J=7.0 \mathrm{~Hz}, 2 \mathrm{H}), 4.07(\mathrm{t}, J=7.3 \mathrm{~Hz}, 2 \mathrm{H}), 4.44(\mathrm{~s}, 2 \mathrm{H}), 8.83(\mathrm{~s}, 1 \mathrm{H}), 9.70$ (s, $1 \mathrm{H}), 9.74(\mathrm{~s}, 1 \mathrm{H}) ;{ }^{13} \mathrm{C} \mathrm{NMR}\left(500 \mathrm{MHz}, \mathrm{CDCl}_{3}\right) \delta$ 11.6, 11.6, 12.0, 16.6, 21.4, 25.6, 25.9, 27.9, 28.3, 32.1, 33.9, 34.0, 46.0, 51.8, 52.0, 52.4, 91.0, 97.9, 99.0, 105.2, 128.4, 129.4, 131.4, 134.1, 135.3, 136.6, 137.6, 137.9, 138.3, 140.0, 149.4, 151.1, 162.0, 172.1, 174.5, 174.7. HRMS (ESI) Calcd for $\mathrm{C}_{37} \mathrm{H}_{46} \mathrm{~N}_{4} \mathrm{O}_{4}$ : 610.3519; found: $611.3610\left(\mathrm{M}+\mathrm{H}^{+}\right)$. 
5-[18-(4-Methoxycarbonyl-butyl)-3,7,8,10,13,13,17-heptamethyl-12,13,22,24-tetrahydroporphin-2-yl]-pentanoic acid methyl ester (8bd). Following the procedure outlined for 8aa, reaction of $\mathbf{6 b}(27 \mathrm{mg}, 0.10 \mathrm{mmol})$ with $7 \mathbf{d}(49 \mathrm{mg}, 0.10 \mathrm{mmol})$ in $\mathrm{CH}_{2} \mathrm{Cl}_{2}(9 \mathrm{~mL})$ and TFA (450 $\mu \mathrm{L})$ gave $\mathbf{8 b c}(41 \mathrm{mg}, 64 \%)$ after chromatography (silica gel, EtOAc:hexanes $=1: 4,1 \% \mathrm{NEt}_{3}$ ). Green solid, mp 135.0-136.0 ${ }^{\circ} \mathrm{C}$. IR(thin film) $3436,1643 \mathrm{~cm}^{-1} ; \mathrm{UV}\left(\mathrm{CH}_{2} \mathrm{Cl}_{2}\right): \lambda_{\max }\left(\varepsilon \mathrm{L} \mathrm{mol}^{-1} \mathrm{~cm}^{-}\right.$ $\left.{ }^{1}\right)=648(26300) \mathrm{nm} ;{ }^{1} \mathrm{H}$ NMR $\left(500 \mathrm{MHz}, \mathrm{CDCl}_{3}\right) \delta-2.47(\mathrm{~s}, 1 \mathrm{H}),-2.05(\mathrm{~s}, 1 \mathrm{H}), 2.02(\mathrm{~s}, 6 \mathrm{H}), 2.05$ (s, 2H), $2.08(\mathrm{~s}, 2 \mathrm{H}), 2.27(\mathrm{~m}, 4 \mathrm{H}), 2.49(\mathrm{~m}, 4 \mathrm{H}), 3.44(\mathrm{~s}, 3 \mathrm{H}), 3.47(\mathrm{~s}, 6 \mathrm{H}), 3.52(\mathrm{~s}, 3 \mathrm{H}), 3.67(\mathrm{~s}$, $6 \mathrm{H}), 3.84$ (s, 3H), 3.88 (t, $J=7.5 \mathrm{~Hz}, 2 \mathrm{H}), 4.00$ (t, $J=7.3 \mathrm{~Hz}, 2 \mathrm{H}), 4.46$ (s, 2H), 8.80 (s, 1H), 9.54 (s, 1H), $9.75(\mathrm{~s}, 1 \mathrm{H}) ;{ }^{13} \mathrm{C}$ NMR $\left(500 \mathrm{MHz}, \mathrm{CDCl}_{3}\right) \delta 11.6,12.1,16.6,21.5,25.4,25.5,26.1,26.4$, $32.1,32.4,32.9,34.4,34.5,46.1,51.7,51.8,52.4,90.9,97.6,99.0,105.1,128.4,129.3,131.3$, 134.0, 135.3, 137.1, 137.3, 137.9, 138.3, 140.4, 149.4, 151.0, 161.8, 172.0, 174.3, 174.5. HRMS (ESI) Calcd for $\mathrm{C}_{39} \mathrm{H}_{50} \mathrm{~N}_{4} \mathrm{O}_{4}$ : 638.3832; found: $639.3901\left(\mathrm{M}+\mathrm{H}^{+}\right)$.

11-[18-(10-Methoxycarbonyl-decyl)-3,7,8,10,13,13,17-heptamethyl-12,13,22,24-tetrahydroporphin-2-yl]-undecanoic acid methyl ester (8be). Following the procedure outlined for 8aa, reaction of $\mathbf{6 b}(27 \mathrm{mg}, 0.10 \mathrm{mmol})$ with $7 \mathbf{e}(66 \mathrm{mg}, 0.10 \mathrm{mmol})$ in $\mathrm{CH}_{2} \mathrm{Cl}_{2}(9 \mathrm{~mL})$ and TFA (450 $\mu \mathrm{L}$ ) gave 8 bc $(55 \mathrm{mg}, 68 \%)$ after chromatography (silica gel, EtOAc:hexanes $\left.=1: 4,1 \% \mathrm{NEt}_{3}\right)$. Green solid, mp 83.0-84.0 ${ }^{\circ} \mathrm{C}$. IR(thin film) $3438,1644 \mathrm{~cm}^{-1}$; UV $\left(\mathrm{CH}_{2} \mathrm{Cl}_{2}\right): \lambda_{\max }\left(\varepsilon \mathrm{L} \mathrm{mol}^{-1} \mathrm{~cm}^{-1}\right)$ $=648(41400) \mathrm{nm} ;{ }^{1} \mathrm{H}$ NMR $\left(500 \mathrm{MHz}, \mathrm{CDCl}_{3}\right) \delta-2.45(\mathrm{~s}, 1 \mathrm{H}),-2.06(\mathrm{~s}, 1 \mathrm{H}), 1.29(\mathrm{br}, 16 \mathrm{H}), 1.48$ (m, 4H), $1.59(\mathrm{~m}, 4 \mathrm{H}), 1.69(\mathrm{~m}, 4 \mathrm{H}), 2.05(\mathrm{~s}, 6 \mathrm{H}), 2.20(\mathrm{~m}, 4 \mathrm{H}), 2.28(\mathrm{~m}, 4 \mathrm{H}), 3.44(\mathrm{~s}, 3 \mathrm{H}), 3.47$ (s, 3H), $3.48(\mathrm{~s}, 3 \mathrm{H}), 3.53(\mathrm{~s}, 3 \mathrm{H}), 3.66(\mathrm{~s}, 6 \mathrm{H}), 3.84(\mathrm{~m}, 2 \mathrm{H}), 3.85(\mathrm{~s}, 3 \mathrm{H}), 3.95(\mathrm{t}, J=7.5 \mathrm{~Hz}, 2 \mathrm{H})$, $4.46(\mathrm{~s}, 2 \mathrm{H}), 8.80(\mathrm{~s}, 1 \mathrm{H}), 9.58(\mathrm{~s}, 1 \mathrm{H}), 9.75(\mathrm{~s}, 1 \mathrm{H}) ;{ }^{13} \mathrm{C}$ NMR $\left(500 \mathrm{MHz}, \mathrm{CDCl}_{3}\right) \delta$ 11.6, 12.1, $16.6,21.5,25.2$, 26.5, 26.8, 29.4, 29.5, 29.6, 29.8, 29.8, 29.9, 30.0, 30.0, 30.1, 30.2, 30.2, 32.1, 33.0, 33.5, 34.4, 46.1, 51.7, 52.4, 90.8, 97.9, 99.0, 105.0, 110.0, 128.2, 129.0, 131.2, 134.3, 135.1, 137.0, 138.0, 138.0, 138.1, 141.2, 149.4, 151.3, 161.6, 171.9, 174.6. HRMS (ESI) Calcd for $\mathrm{C}_{51} \mathrm{H}_{74} \mathrm{~N}_{4} \mathrm{O}_{4}: 806.5710$; found: $807.5786\left(\mathrm{M}+\mathrm{H}^{+}\right)$.

$\mathbf{2 , 2 , 7 , 8 , 1 2 , 1 3 , 1 7 , 1 8 - O c t a m e t h y l - 2 , 3 , 2 2 , 2 4 - t e t r a h y d r o - p o r p h i n e ~ ( 8 c a ) . ~ F o l l o w i n g ~ t h e ~ p r o c e d u r e ~}$ outlined for 8aa, reaction of $\mathbf{6 c}(20 \mathrm{mg}, 78 \mu \mathrm{mol})$ with $7 \mathbf{a}(23 \mathrm{mg}, 78 \mu \mathrm{mol})$ in $\mathrm{CH}_{2} \mathrm{Cl}_{2}(7 \mathrm{~mL})$ and TFA $(350 \mu \mathrm{L})$ gave $8 \mathbf{c a}(19 \mathrm{mg}, 58 \%)$ after chromatography (silica gel, EtOAc:hexanes $=1: 4,1 \%$ $\mathrm{NEt}_{3}$ ). The chlorin was identical to the literature compound. ${ }^{1}$

3-[18-(2-Methoxycarbonyl-ethyl)-3,7,8,13,13,17-hexamethyl-12,13,22,24-tetrahydro-porphin2-yl]-propionic acid methyl ester (8cb). Following the procedure outlined for 8aa, reaction of 6b $(25 \mathrm{mg}, 96 \mu \mathrm{mol})$ with $\mathbf{7 b}(42 \mathrm{mg}, 96 \mu \mathrm{mol})$ in $\mathrm{CH}_{2} \mathrm{Cl}_{2}(8.8 \mathrm{~mL})$ and TFA $(440 \mu \mathrm{L})$ gave 8cb (34 mg, 63\%) after chromatography (silica gel, EtOAc:hexanes $=1: 4,1 \% \mathrm{NEt}_{3}$ ). To quench the reaction, $3 \mathrm{M} \mathrm{NH} \mathrm{H}_{4} \mathrm{OH}$ was substituted for saturated aq $\mathrm{KHCO}_{3}$. The chlorin was identical to the literature compound. ${ }^{1}$

4-[18-(3-Methoxycarbonyl-propyl)-3,7,8,13,13,17-hexamethyl-12,13,22,24-tetrahydro-porphin-2-yl]-butyric acid methyl ester $(8 \mathrm{cc})$. Following the procedure outlined for 8aa, reaction of 6c $(34 \mathrm{mg}, 0.13 \mathrm{mmol})$ with $7 \mathbf{c}(61 \mathrm{mg}, 0.13 \mathrm{mmol})$ in $\mathrm{CH}_{2} \mathrm{Cl}_{2}(12 \mathrm{~mL})$ and TFA $(600 \mu \mathrm{L})$ gave $8 \mathbf{c c}(45 \mathrm{mg}, 57 \%)$ after chromatography (silica gel, EtOAc:hexanes $\left.=1: 4,1 \% \mathrm{NEt}_{3}\right)$. Green solid, $\mathrm{mp}$ 192.0-194.0 ${ }^{\circ} \mathrm{C}$. IR(thin film) 3422, $1641 \mathrm{~cm}^{-1}$; UV $\left(\mathrm{CH}_{2} \mathrm{Cl}_{2}\right): \lambda_{\max }\left(\varepsilon \mathrm{L} \mathrm{mol}^{-1} \mathrm{~cm}^{-1}\right)=$ 643(52000) nm; ${ }^{1} \mathrm{H}$ NMR (500 MHz, $\left.\mathrm{CDCl}_{3}\right) \delta-2.46(\mathrm{~s}, 2 \mathrm{H}), 2.07,(\mathrm{~s}, 6 \mathrm{H}), 2.55(\mathrm{~m}, 4 \mathrm{H}), 2.68(\mathrm{~m}$, $4 \mathrm{H}), 3.41(\mathrm{~s}, 3 \mathrm{H}), 3.43(\mathrm{~s}, 3 \mathrm{H}), 3.48(\mathrm{~s}, 3 \mathrm{H}), 3.51(\mathrm{~s}, 3 \mathrm{H}), 3.71(\mathrm{~m}, 6 \mathrm{H}), 3.94(\mathrm{t}, J=7.5 \mathrm{~Hz}, 2 \mathrm{H})$, 
$4.09(\mathrm{t}, J=7.3 \mathrm{~Hz}, 2 \mathrm{H}), 4.64(\mathrm{~s}, 2 \mathrm{H}), 8.80(\mathrm{~s}, 1 \mathrm{H}), 8.91(\mathrm{~s}, 1 \mathrm{H}), 9.69(\mathrm{~s}, 1 \mathrm{H}), 9.81(\mathrm{~s}, 1 \mathrm{H}) ;{ }^{13} \mathrm{C}$ NMR $\left(500 \mathrm{MHz}, \mathrm{CDCl}_{3}\right) \delta 11.5,11.5,11.7,12.0,25.6,25.9,27.9,28.4,30.0,31.6,33.8,33.9$, $46.7,51.7,51.8,52.5,91.0,93.1,98.7,99.6,128.7,129.1,132.5,133.8,133.9,136.8,137.2$, 138.5, 138.7, 140.5, 149.5, 150.7, 162.2, 173.6, 174.4, 174.7. HRMS (ESI) Calcd for $\mathrm{C}_{36} \mathrm{H}_{44} \mathrm{~N}_{4} \mathrm{O}_{4}$ : 596.3363; found: $597.3424\left(\mathrm{M}+\mathrm{H}^{+}\right)$.

5-[18-(4-Methoxycarbonyl-butyl)-3,7,8,13,13,17-hexamethyl-12,13,22,24-tetrahydro-porphin-2-yl]-pentanoic acid methyl ester (8cd). Following the procedure outlined for 8aa, reaction of $6 \mathbf{c}(26 \mathrm{mg}, 0.10 \mathrm{mmol})$ with $7 \mathbf{d}(49 \mathrm{mg}, 0.10 \mathrm{mmol})$ in $\mathrm{CH}_{2} \mathrm{Cl}_{2}(9 \mathrm{~mL})$ and TFA $(450 \mu \mathrm{L})$ gave 8cd (32 mg, 51\%) after chromatography (silica gel, EtOAc:hexanes $\left.=1: 4,1 \% \mathrm{NEt}_{3}\right)$. Green solid, mp 168.0-169.0 ${ }^{\circ} \mathrm{C}$. IR(thin film) 3444, $1641 \mathrm{~cm}^{-1} ; \mathrm{UV}\left(\mathrm{CH}_{2} \mathrm{Cl}_{2}\right): \lambda_{\max }\left(\varepsilon \mathrm{L} \mathrm{mol} \mathrm{cm}^{-1}\right)=$ 643(40900) nm; ${ }^{1} \mathrm{H}$ NMR $\left(500 \mathrm{MHz}, \mathrm{CDCl}_{3}\right) \delta-2.46(\mathrm{~s}, 2 \mathrm{H}), 2.06(\mathrm{~m}, 4 \mathrm{H}), 2.07(\mathrm{~s}, 6 \mathrm{H}), 2.26(\mathrm{~m}$, $4 \mathrm{H}), 2.49(\mathrm{~m}, 4 \mathrm{H}), 3.41,(\mathrm{~s}, 3 \mathrm{H}), 3.44(\mathrm{~s}, 3 \mathrm{H}), 3.48(\mathrm{~s}, 3 \mathrm{H}), 3.51(\mathrm{~s}, 3 \mathrm{H}), 3.66(\mathrm{~s}, 6 \mathrm{H}), 3.90(\mathrm{t}$, $J=7.5 \mathrm{~Hz}, 2 \mathrm{H}), 4.03(\mathrm{t}, J=7.5 \mathrm{~Hz}, 2 \mathrm{H}), 4.65(\mathrm{~s}, 2 \mathrm{H}), 8.81(\mathrm{~s}, 1 \mathrm{H}), 8.91(\mathrm{~s}, 1 \mathrm{H}), 9.68(\mathrm{~s}, 1 \mathrm{H}), 9.69$ $(\mathrm{s}, 1 \mathrm{H}) ;{ }^{13} \mathrm{C}$ NMR $\left(500 \mathrm{MHz}, \mathrm{CDCl}_{3}\right) \delta 11.6,12.1,19.0,24.9,25.4,25.5,26.2,26.5,27.0,31.7$, $32.5,33.0,34.4,34.5,46.7,51.7,51.8,52.5,91.0,93.1,98.7,99.2,128.5,129.0,132.5,133.8$, $133.8,136.6,137.7,138.4,138.7,141.0,149.5,150.7,162.1,173.5,174.3,174.5$. HRMS (ESI) Calcd for $\mathrm{C}_{38} \mathrm{H}_{48} \mathrm{~N}_{4} \mathrm{O}_{4}$ : 624.3676; found: $625.3746\left(\mathrm{M}+\mathrm{H}^{+}\right)$.

\section{5-[10-Decyl-18-(4-methoxycarbonyl-butyl)-3,7,8,13,13,17-hexamethyl-12,13,22,24-tetra-}

hydro-porphin-2-yl]-pentanoic acid methyl ester (8ce). Following the procedure outlined for 8aa, reaction of 6c $(20 \mathrm{mg}, 77 \mu \mathrm{mol})$ with $7 \mathbf{e}(46 \mathrm{mg}, 77 \mu \mathrm{mol})$ in $\mathrm{CH}_{2} \mathrm{Cl}_{2}(8 \mathrm{~mL})$ and TFA (400 $\mu \mathrm{L})$ gave 8ce (30 mg, 55\%) after chromatography (silica gel, EtOAc:hexanes $=1: 4,1 \% \mathrm{NEt}_{3}$ ). Green solid, mp 123.0-124.0 ${ }^{\circ} \mathrm{C}$. IR(thin film) $3438,1639 \mathrm{~cm}^{-1} ; \mathrm{UV}\left(\mathrm{CH}_{2} \mathrm{Cl}_{2}\right): \lambda_{\max }\left(\varepsilon \mathrm{L} \mathrm{mol}^{-1} \mathrm{~cm}^{-}\right.$ $\left.{ }^{1}\right)=643(32000) \mathrm{nm} ;{ }^{1} \mathrm{H}$ NMR $\left(500 \mathrm{MHz}, \mathrm{CDCl}_{3}\right) \delta-2.45(\mathrm{~s}, 2 \mathrm{H}), 1.29(\mathrm{br}, 16 \mathrm{H}), 1.49(\mathrm{~m}, 4 \mathrm{H})$, $1.60(\mathrm{~m}, 4 \mathrm{H}), 1.69(\mathrm{~m}, 4 \mathrm{H}), 2.07(\mathrm{~s}, 6 \mathrm{H}), 2.22(\mathrm{~m}, 4 \mathrm{H}), 2.28(\mathrm{~m}, 4 \mathrm{H}), 3.41(\mathrm{~s}, 3 \mathrm{H}), 3.44(\mathrm{~s}, 3 \mathrm{H})$, $3.48(\mathrm{~s}, 3 \mathrm{H}), 3.52(\mathrm{~s}, 3 \mathrm{H}), 3.66(\mathrm{~s}, 6 \mathrm{H}), 3.87(\mathrm{t}, J=7.8 \mathrm{~Hz}, 2 \mathrm{H}), 4.00(\mathrm{t}, J=7.3 \mathrm{~Hz}, 2 \mathrm{H}), 4.65(\mathrm{~s}, 2 \mathrm{H})$, $7.80(\mathrm{~s}, 1 \mathrm{H}), 8.90(\mathrm{~s}, 1 \mathrm{H}), 9.70(\mathrm{~s}, 1 \mathrm{H}), 9.73(\mathrm{~s}, 1 \mathrm{H}) ;{ }^{13} \mathrm{C} \mathrm{NMR}\left(500 \mathrm{MHz}, \mathrm{CDCl}_{3}\right) \delta 11.5,11.6$, $11.7,12.0,25.2,26.5,26.8,29.4,29.4,29.5,29.6,29.8,29.8,29.9,30.0,30.0,30.1,30.2,30.3$, $31.7,33.1,33.6,34.4,46.7,51.7,52.5,90.8,93.0,98.8,98.8,99.6,128.3,128.8,132.7,133.6$, 133.7, 136.3, 138.2, 138.5, 138.7, 141.9, 149.9, 150.8, 161.8, 173.4, 174.6. HRMS (ESI) Calcd for $\mathrm{C}_{50} \mathrm{H}_{72} \mathrm{~N}_{4} \mathrm{O}_{4}$ : 792.5554; found: $793.5632\left(\mathrm{M}+\mathrm{H}^{+}\right)$.

$\mathbf{2 , 2 , 7 , 8 , 1 2 , 1 3 , 1 7 , 1 8 - O c t a m e t h y l - 5 - p e n t y l - 2 , 3 , 2 2 , 2 4 - t e t r a h y d r o - p o r p h i n e ~ ( 8 d a ) . ~ N i t r o g e n ~ w a s ~}$ bubbled through a suspension of $\mathbf{6 d}(43 \mathrm{mg}, 0.13 \mathrm{mmol})$ and $7 \mathbf{a}(35 \mathrm{mg}, 0.12 \mathrm{mmol})$ in $\mathrm{CH}_{2} \mathrm{Cl}_{2}(14$ $\mathrm{mL}$ ) for $10 \mathrm{~min}$. The mixture was treated with $p$-toluenesulfonic acid (147 $\mathrm{mg}, 0.77 \mathrm{mmol})$ in $\mathrm{MeOH}(1.0 \mathrm{~mL})$ and stirred at $\mathrm{rt}$ in the dark for $2 \mathrm{~h}$. The reaction was then poured into saturated aq $\mathrm{KHCO}_{3}(25 \mathrm{~mL})$. The layers were separated, and the aqueous layer was extracted with $\mathrm{CH}_{2} \mathrm{Cl}_{2}(15$ $\mathrm{mL}$ ). The combined organic layers were washed sequentially with water and brine, dried over $\mathrm{Na}_{2} \mathrm{SO}_{4}$, filtered, and concentrated. The residue was purified by chromatography (silica gel, EtOAc:hexanes $\left.=1: 4,1 \% \mathrm{NEt}_{3}\right)$ to give $\mathbf{8 d a}(23 \mathrm{mg}, 39 \%)$ as a green solid, $\mathrm{mp}$ decomp $>250{ }^{\circ} \mathrm{C}$. $\mathrm{R}_{f}$ (1:4 EtOAc/hexanes) 0.60; IR(thin film) 3293, $1602 \mathrm{~cm}^{-1} ;{ }^{1} \mathrm{H} \mathrm{NMR}\left(500 \mathrm{MHz}, \mathrm{CDCl}_{3}\right)-2.42$ (s, $1 \mathrm{H}),-1.95(\mathrm{~s}, 1 \mathrm{H}), 1.1(\mathrm{t}, \mathrm{J}=7.3,3 \mathrm{H}), 1.62(\mathrm{~m}, 2 \mathrm{H}), 1.83(\mathrm{~m}, 2 \mathrm{H}), 2.10(\mathrm{~s}, 6 \mathrm{H}), 2.15(\mathrm{~m}, 2 \mathrm{H}), 3.40$ $(\mathrm{s}, 3 \mathrm{H}), 3.44(\mathrm{~s}, 3 \mathrm{H}), 3.47(\mathrm{~s}, 3 \mathrm{H}), 3.49(\mathrm{~s}, 3 \mathrm{H}), 3.50(\mathrm{~s}, 3 \mathrm{H}), 3.55(\mathrm{~s}, 3 \mathrm{H}), 4.26(\mathrm{br} \mathrm{s}, 2 \mathrm{H}), 4.55(\mathrm{~s}$, 2H), $8.83(\mathrm{~s}, 1 \mathrm{H}), 9.57(\mathrm{~s}, 1 \mathrm{H}), 9.76(\mathrm{~s}, 1 \mathrm{H}) ;{ }^{13} \mathrm{C} \mathrm{NMR}\left(500 \mathrm{MHz}, \mathrm{CDCl}_{3}\right) \delta$ 11.5, 11.9, 12.0, 12.2, $14.6,15.5,23.1,29.9,31.8,33.0,33.4,34.3,45.9,51.1,90.7,97.5,98.9,111.2,127.4,129.3$, $130.9,132.9,134.8,135.6,136.0,137.2,137.6,137.8,149.5,152.1,161.5,171.6$; UV $\left(\mathrm{CHCl}_{3}\right)$ : 
$\lambda_{\max }\left(\varepsilon \mathrm{L} \mathrm{mol}^{-1} \mathrm{~cm}^{-1}\right)=399$ (170700), 499 (16500), 649 (45500) nm; HRMS (EI) Calcd for $\mathrm{C}_{33} \mathrm{H}_{42} \mathrm{~N}_{4}$ : 494.3409; found: 494.3409 .

3-[18-(2-Methoxycarbonyl-ethyl)-3,7,8,13,13,17-hexamethyl-10-pentyl-12,13,22,24-tetrahydro-porphin-2-yl]-propionic acid methyl ester $(\mathbf{8 d b})$. Following the procedure outlined for 8da, reaction of $\mathbf{6 d}(29 \mathrm{mg}, 87 \mu \mathrm{mol})$ and $7 \mathbf{b}(35 \mathrm{mg}, 79 \mu \mathrm{mol})$ in $\mathrm{CH}_{2} \mathrm{Cl}_{2}(10 \mathrm{~mL})$ with $p$ toluenesulfonic acid $(99 \mathrm{mg}, 0.52 \mathrm{mmol})$ in $\mathrm{MeOH}(1.0 \mathrm{~mL})$ gave $8 \mathrm{db}(26 \mathrm{mg}, 51 \%)$ after chromatography (silica gel, EtOAc:hexanes $\left.=1: 9,1 \% \mathrm{NEt}_{3}\right)$. Green solid, mp 205-206 ${ }^{\circ} \mathrm{C} . \mathrm{R}_{f}(1: 4$ EtOAc/hexanes) 0.50; IR(thin film) 3300, $1736 \mathrm{~cm}^{-1} ;{ }^{1} \mathrm{H}$ NMR (500 MHz, $\left.\mathrm{CDCl}_{3}\right)-2.50(\mathrm{~s}, 1 \mathrm{H})$, $-1.95(\mathrm{~s}, 1 \mathrm{H}), 1.11(\mathrm{t}, J=7 \mathrm{H}, 3 \mathrm{H}), 1.63(\mathrm{~m}, 2 \mathrm{H}) 1.82(\mathrm{~m}, 2 \mathrm{H}), 2.10(\mathrm{~s}, 6 \mathrm{H}), 2.14(\mathrm{~m}, 2 \mathrm{H}), 3.27(\mathrm{~m}$, $4 \mathrm{H}), 3.49(\mathrm{~s}, 3 \mathrm{H}), 3.50(\mathrm{~s}, 3 \mathrm{H}), 3.54(\mathrm{~s}, 3 \mathrm{H}), 3.55(\mathrm{~s}, 3 \mathrm{H}), 3.75(\mathrm{~s}, 3 \mathrm{H}), 3.76(\mathrm{~s}, 3 \mathrm{H}), 4.25(\mathrm{~m}, 4 \mathrm{H})$, $4.37(\mathrm{t}, J=7.63,2 \mathrm{H}), 4.55(\mathrm{~s}, 2 \mathrm{H}), 8.88(\mathrm{~s}, 1 \mathrm{H}), 9.64(\mathrm{~s}, 1 \mathrm{H}), 9.78(\mathrm{~s}, 1 \mathrm{H}),{ }^{13} \mathrm{C}$ NMR $(500 \mathrm{MHz}$, $\left.\mathrm{CDCl}_{3}\right) \delta 11.5,11.9,12.1,14.5,15.5,21.8,22.1,23.1,31.8,32.9,33.3,34.3,36.9,37.4,45.9$, 51.2, 51.9, 52.0, 91.1, 97.5, 98.8, 111.4, 128.0, 129.6, 131.3, 133.5, 135.3, 136.0, 137.5, 137.8, 138.1, 138.8, 149.3, 150.4, 162.3, 171.7, 173.7, 174.2; UV $\left(\mathrm{CHCl}_{3}\right): \lambda_{\max }\left(\varepsilon \mathrm{L} \mathrm{mol}^{-1} \mathrm{~cm}^{-1}\right) 504$ (8700), 594 (3300), 648 (18300) nm; HRMS (EI) Calcd for $\mathrm{C}_{39} \mathrm{H}_{50} \mathrm{~N}_{4} \mathrm{O}_{4}$ : 638.3832; found: 638.3822 .

4-[18-(3-Methoxycarbonyl-propyl)-3,7,8,13,13,17-hexamethyl-10-pentyl-12,13,22,24-tetrahydro-porphin-2-yl]-butyric acid methyl ester (8dc). Following the procedure outlined for 8aa, reaction of $\mathbf{6 d}(12 \mathrm{mg}, 35 \mu \mathrm{mol})$ with $7 \mathbf{c}(16 \mathrm{mg}, 35 \mu \mathrm{mol})$ in $\mathrm{CH}_{2} \mathrm{Cl}_{2}(3.2 \mathrm{~mL})$ and TFA $(160 \mu \mathrm{L})$ gave 8dc (11 mg, 49\%) as a green film after chromatography (silica gel, EtOAc:hexanes $=1: 4,1 \%$ $\left.\mathrm{NEt}_{3}\right) . \mathrm{R}_{f}\left(1: 4 \mathrm{EtOAc} /\right.$ hexanes) 0.25 ; IR(thin film) 1734, 1603, 1436, $1157 \mathrm{~cm}^{-1} ; \mathrm{UV}\left(\mathrm{CH}_{2} \mathrm{Cl}_{2}\right)$ : $\lambda_{\max }\left(\varepsilon \mathrm{L} \mathrm{mol}^{-1} \mathrm{~cm}^{-1}\right)=648(62500), 594,502,399 \mathrm{~nm} ;{ }^{1} \mathrm{H} \mathrm{NMR}\left(500 \mathrm{MHz}, \mathrm{CDCl}_{3}\right) \delta-2.46(\mathrm{br} \mathrm{s}$, $1 \mathrm{H}),-1.99(\mathrm{br} \mathrm{s}, 1 \mathrm{H}), 1.06(\mathrm{t}, J=7.2,3 \mathrm{H}), 1.59(\mathrm{~m}, 2 \mathrm{H}), 1.81(\mathrm{~m}, 2 \mathrm{H}), 2.06(\mathrm{~s}, 6 \mathrm{H}), 2.12,(\mathrm{~m}, 2 \mathrm{H})$, $2.55(\mathrm{~m}, 4 \mathrm{H}), 2.68(\mathrm{~m}, 4 \mathrm{H}), 3.44(\mathrm{~s}, 3 \mathrm{H}), 3.48(\mathrm{~s}, 3 \mathrm{H}), 3.52(\mathrm{~s}, 3 \mathrm{H}), 3.56(\mathrm{~s}, 3 \mathrm{H}), 3.71(\mathrm{~s}, 3 \mathrm{H}), 3.72$ (s, 3H), $3.91(\mathrm{t}, J=7.5,2 \mathrm{H}), 4.05(\mathrm{t}, J=7.6,2 \mathrm{H}), 4.28(\mathrm{~m}, 2 \mathrm{H}), 4.55(\mathrm{~s}, 2 \mathrm{H}), 8.82(\mathrm{~s}, 1 \mathrm{H}), 9.6(\mathrm{~s}$, 1H), $9.77(\mathrm{~s}, 1 \mathrm{H}) ;{ }^{13} \mathrm{C} \mathrm{NMR}\left(500 \mathrm{MHz}, \mathrm{CDCl}_{3}\right) \delta 11.6,12.0,12.3,14.6,15.6,23.1,25.5,25.9$, $27.8,28.3,31.9,33.0,33.5,33.8,33.9,34.3,45.9,51.3,51.7,51.8,91.0,98.0,98.9,111.4,127.8$, $129.4,131.2,134.2,135.9,136.5,137.6,137.7,138.0,139.8,149.4,151.2,162.0,171.8,174.4$, 174.7. HRMS (EI) Calcd for $\mathrm{C}_{41} \mathrm{H}_{54} \mathrm{~N}_{4} \mathrm{O}_{4}$ : 666.4145; found: 666.4152 .

5-[18-(4-Methoxycarbonyl-butyl)-3,7,8,13,13,17-hexamethyl-10-pentyl-12,13,22,24-tetrahydro-porphin-2-yl]-pentanoic acid methyl ester (8dd). Following the procedure outlined for 8aa, reaction of $\mathbf{6 d}(12 \mathrm{mg}, 35 \mu \mathrm{mol})$ with $7 \mathbf{d}(17 \mathrm{mg}, 35 \mu \mathrm{mol})$ in $\mathrm{CH}_{2} \mathrm{Cl}_{2}(3.2 \mathrm{~mL})$ and TFA (160 $\mu \mathrm{L})$ gave 8dd (12 mg, 49\%) as a green film after chromatography (silica gel, EtOAc:hexanes = $\left.1: 4,1 \% \mathrm{NEt}_{3}\right) . \mathrm{R}_{f}\left(1: 4 \mathrm{EtOAc} /\right.$ hexanes) 0.23 ; IR(thin film) $1734,1603,1147 \mathrm{~cm}^{-1} ; \mathrm{UV}\left(\mathrm{CH}_{2} \mathrm{Cl}_{2}\right)$ : $\lambda_{\max }\left(\varepsilon \mathrm{L} \mathrm{mol}^{-1} \mathrm{~cm}^{-1}\right)=648(63100), 596,501,399 \mathrm{~nm} ;{ }^{1} \mathrm{H}$ NMR $\left(500 \mathrm{MHz}, \mathrm{CDCl}_{3}\right) \delta-2.47$ (br s, $1 \mathrm{H}),-2.00($ br s, $1 \mathrm{H}), 1.06(\mathrm{t}, J=7.2,3 \mathrm{H}), 1.59(\mathrm{~m}, 2 \mathrm{H}), 1.80(\mathrm{~m}, 2 \mathrm{H}), 2.01-2.06$ (overlapping singlet and multiplet, $10 \mathrm{H}), 2.12(\mathrm{~m}, 2 \mathrm{H}), 2.24(\mathrm{~m}, 4 \mathrm{H}), 2.48(\mathrm{t}, J=7.5,4 \mathrm{H}), 3.44(\mathrm{~s}, 3 \mathrm{H}), 3.47(\mathrm{~s}$, $3 \mathrm{H}), 3.52(\mathrm{~s}, 3 \mathrm{H}), 3.56(\mathrm{~s}, 3 \mathrm{H}), 3.65$ (overlapping singlets, $6 \mathrm{H}), 3.88(\mathrm{t}, J=7.5,2 \mathrm{H}), 3.99(\mathrm{t}, J=$ 7.6, 2H), $4.28(\mathrm{~m}, 2 \mathrm{H}), 4.54(\mathrm{~s}, 2 \mathrm{H}), 8.82(\mathrm{~s}, 1 \mathrm{H}), 9.53(\mathrm{~s}, 1 \mathrm{H}), 9.77(\mathrm{~s}, 1 \mathrm{H}) ;{ }^{13} \mathrm{C} \mathrm{NMR}(500 \mathrm{MHz}$, $\left.\mathrm{CDCl}_{3}\right) \delta 11.6,12.1,12.3,14.6,15.6,23.1,25.4,25.5,26.1,26.4,31.9,32.4,32.8,33.0,33.5$, $34.3,34.4,34.5,45.9,51.2,51.7,51.8,90.9,97.7,98.9,111.3,127.7,129.3,131.2,134.1,135.9$, 137.1, 137.4, 137.7, 137.9, 140.4, 149.4, 151.1, 161.9, 171.7, 174.3, 174.5. HRMS (EI) Calcd for $\mathrm{C}_{43} \mathrm{H}_{58} \mathrm{~N}_{4} \mathrm{O}_{4}$ : 694.4458; found: 694.4443 . 
11-[18-(10-Methoxycarbonyl-decyl)-3,7,8,13,13,17-hexamethyl-10-pentyl-12,13,22,24-tetrahydro-porphin-2-yl]-undecanoic acid methyl ester (8de). Following the procedure outlined for 8aa, reaction of $\mathbf{6 d}(12 \mathrm{mg}, 35 \mu \mathrm{mol})$ with $7 \mathbf{e}(23 \mathrm{mg}, 35 \mu \mathrm{mol})$ in $\mathrm{CH}_{2} \mathrm{Cl}_{2}(3.2 \mathrm{~mL})$ and TFA (160 $\mu \mathrm{L})$ gave 8de $(14 \mathrm{mg}, 47 \%)$ as a green film after chromatography (silica gel, EtOAc:hexanes $=$ $\left.1: 4,1 \% \mathrm{NEt}_{3}\right) . \mathrm{R}_{f}\left(1: 4 \mathrm{EtOAc} /\right.$ hexanes) $0.40 ; \mathrm{IR}$ (thin film) $1740,1603,1109 \mathrm{~cm}^{-1} ; \mathrm{UV}\left(\mathrm{CH}_{2} \mathrm{Cl}_{2}\right)$ : $\lambda_{\max }\left(\varepsilon \mathrm{L} \mathrm{mol}^{-1} \mathrm{~cm}^{-1}\right)=648(68100), 594,501,398 \mathrm{~nm} ;{ }^{1} \mathrm{H} \mathrm{NMR}\left(500 \mathrm{MHz}, \mathrm{CDCl}_{3}\right) \delta-2.44(\mathrm{br} \mathrm{s}$, $1 \mathrm{H}),-2.00$ (br s, $1 \mathrm{H}), 1.07(\mathrm{t}, J=7.3,3 \mathrm{H}), 1.29$ (br m, $16 \mathrm{H}), 1.47(\mathrm{~m}, 4 \mathrm{H}), 1.6(\mathrm{~m}, 6 \mathrm{H}), 1.7(\mathrm{~m}$, $4 \mathrm{H}), 1.81(\mathrm{~m}, 2 \mathrm{H}), 2.06(\mathrm{~s}, 6 \mathrm{H}), 2.09-2.24(\mathrm{br} \mathrm{m}, 6 \mathrm{H}), 2.28(\mathrm{t}, J=7.6,4 \mathrm{H}), 3.44(\mathrm{~s}, 3 \mathrm{H}), 3.47(\mathrm{~s}$, $3 \mathrm{H}), 3.52(\mathrm{~s}, 3 \mathrm{H}), 3.57(\mathrm{~s}, 3 \mathrm{H}), 3.66$ (overlapping singlets, $6 \mathrm{H}), 3.83(\mathrm{t}, J=7.5,2 \mathrm{H}), 3.95(\mathrm{t}, J=$ $7.5,2 \mathrm{H}), 4.28(\mathrm{~m}, 2 \mathrm{H}), 4.55(\mathrm{~s}, 2 \mathrm{H}), 8.82(\mathrm{~s}, 1 \mathrm{H}), 9.57(\mathrm{~s}, 1 \mathrm{H}), 9.78(\mathrm{~s}, 1 \mathrm{H}) ;{ }^{13} \mathrm{C} \mathrm{NMR}(500 \mathrm{MHz}$, $\left.\mathrm{CDCl}_{3}\right) \delta 11.6,12.0,12.3,14.6,15.6,23.1,25.20,25.21,26.4,26.8,29.40,29.41,29.53,29.55,29.79$, 29.80, 29.9, 29.96, 30.02, 30.1, 30.16, 30.23 31.9, 33.02, 33.03, 33.49, 33.51, 34.4, 45.9, 51.2, 51.7, 90.8, 98.0, 98.9, 111.2, 127.5, 129.0, 131.1, 134.3, 135.7, 137.1, 137.7, 137.9, 138.0, 141.2, 149.4, 151.4, 161.6, 171.6, 174.6. HRMS (EI) Calcd for $\mathrm{C}_{55} \mathrm{H}_{82} \mathrm{~N}_{4} \mathrm{O}_{4}$ : 862.6336; found: 862.6320.

5-Decyl-2,2,7,8,12,13,17,18-octamethyl-2,3,22,24-tetrahydro-porphine (8ea). A solution of 6e (24 mg, $60 \mu \mathrm{mol}$ ) and 3,4,3',4'-tetramethyl-1H,1'H-2,2'-methanediyl-bis-pyrrole ${ }^{4}(9.3 \mathrm{mg}, 46$ $\mu \mathrm{mol})$ in $\mathrm{CH}_{2} \mathrm{Cl}_{2}(1.7 \mathrm{~mL})$ was added dropwise to a solution of $p$-toluenesulfonic acid (57 $\mathrm{mg}$, $0.45 \mathrm{mmol})$ in $\mathrm{MeOH} / \mathrm{CH}_{2} \mathrm{Cl}_{2}(400 \mu \mathrm{L} / 3.8 \mathrm{~mL})$ over a period of $2 \mathrm{~h}$. The solution was stirred at $\mathrm{rt}$ in the dark for $2 \mathrm{~h}$ and then poured into saturated aq $\mathrm{Na}_{2} \mathrm{CO}_{3}(15 \mathrm{~mL})$. The layers were separated, and the aqueous layer was extracted with $\mathrm{CH}_{2} \mathrm{Cl}_{2}(10 \mathrm{~mL})$. The combined organic layers were washed sequentially with water and brine, dried over $\mathrm{Na}_{2} \mathrm{SO}_{4}$, filtered, and concentrated. The residue was purified by chromatography ( (9.9 mg, 38\%) as a green solid, mp 134-135 ${ }^{\circ} \mathrm{C}$. $\mathrm{R}_{f}\left(1: 4\right.$ EtOAc/hexanes) $0.57 ;{ }^{1} \mathrm{H}$ NMR (500 $\left.\mathrm{MHz}, \mathrm{CDCl}_{3}\right)-2.45$ (br s, $\left.1 \mathrm{H}\right),-1.89$ (br s, $\left.1 \mathrm{H}\right), 0.94(\mathrm{t}, J=6.9,3 \mathrm{H}), 1.50-1.25(\mathrm{~m}, 10 \mathrm{H}), 1.56(\mathrm{~m}$, 2H), $1.82(\mathrm{~m}, 2 \mathrm{H}), 2.07$ (s, 6H), 2.18-2.04 (m, 2H), $3.38(\mathrm{~s}, 3 \mathrm{H}), 3.42(\mathrm{~s}, 3 \mathrm{H}), 3.45(\mathrm{~s}, 3 \mathrm{H}), 3.48(\mathrm{~s}$, $3 \mathrm{H}), 3.50(\mathrm{~s}, 3 \mathrm{H}), 3.55(\mathrm{~s}, 3 \mathrm{H}), 4.3-4.2(\mathrm{~m}, 2 \mathrm{H}), 4.54(\mathrm{~s}, 2 \mathrm{H}), 8.80(\mathrm{~s}, 1 \mathrm{H}), 9.55(\mathrm{~s}, 1 \mathrm{H}), 9.75(\mathrm{~s}, 1 \mathrm{H})$; ${ }^{13} \mathrm{C}$ NMR $\left(500 \mathrm{MHz}, \mathrm{CDCl}_{3}\right) \delta 11.52,11.53,11.9,12.0,12.2,14.4,15.6,22.9,29.6,29.9,30.0$, $30.1,30.77,30.80,32.2,33.5,34.6,45.9,51.1,90.7,97.5,98.9,111.2,127.4,129.4,130.9,132.9$, 134.8, 135.6, 136.0, 137.2, 137.6, 137.8, 149.4, 152.1, 161.5, 171.6; UV $\left(\mathrm{CHCl}_{3}\right): \lambda_{\max }\left(\varepsilon \mathrm{L} \mathrm{mol}^{-1}\right.$, $\mathrm{cm}^{-1}$ ) = 399 (169500), 499 (17400), 649 (45900) nm; HRMS (EI) Calcd for $\mathrm{C}_{38} \mathrm{H}_{52} \mathrm{~N}_{4}$ : 564.4192; found: 564.4184 .

3-[10-Decyl-18-(2-methoxycarbonyl-ethyl)-3,7,8,13,13,17-hexamethyl-12,13,22,24-tetrahydro-porphin-2-yl]-propionic acid methyl ester (8eb). Following the procedure outlined for 8da, reaction of $\mathbf{6 e}(23 \mathrm{mg}, 58 \mu \mathrm{mol})$ and $\mathbf{7 b}(24 \mathrm{mg}, 55 \mu \mathrm{mol})$ in $\mathrm{CH}_{2} \mathrm{Cl}_{2}(4.7 \mathrm{~mL})$ with $p$ toluenesulfonic acid $(67 \mathrm{mg}, 0.35 \mathrm{mmol})$ in $\mathrm{MeOH}(0.4 \mathrm{~mL})$ gave 8eb $(16 \mathrm{mg}, 42 \%)$ after chromatography (silica gel, EtOAc:hexanes $=1: 9,1 \% \mathrm{NEt}_{3}$ ). Green solid, mp 190-191 ${ }^{\circ} \mathrm{C} . \mathrm{R}_{f}$ (2:3 EtOAc/hexanes) 0.63; IR(thin film) 3277, $1735 \mathrm{~cm}^{-1} ;{ }^{1} \mathrm{H}$ NMR (500 MHz, $\left.\mathrm{CDCl}_{3}\right)-2.47$ (br s, $1 \mathrm{H}),-1.96(\mathrm{br} \mathrm{s}, 1 \mathrm{H}), 0.95(\mathrm{t}, J=7.0,3 \mathrm{H}), 1.5-1.3(\mathrm{~m}, 10 \mathrm{H}), 1.57(\mathrm{~m}, 2 \mathrm{H}), 1.83(\mathrm{~m}, 2 \mathrm{H}), 2.08(\mathrm{~s}$, $6 \mathrm{H}), 2.13(\mathrm{~m}, 2 \mathrm{H}), 3.26-3.20(\mathrm{~m}, 4 \mathrm{H}), 3.47(\mathrm{~s}, 3 \mathrm{H}), 3.51(\mathrm{~s}, 3 \mathrm{H}), 3.52(\mathrm{~s}, 3 \mathrm{H}), 3.56(\mathrm{~s}, 3 \mathrm{H}), 3.72(\mathrm{~s}$, $3 \mathrm{H}), 3.73(\mathrm{~s}, 3 \mathrm{H}), 4.22(\mathrm{t}, J=7.9,2 \mathrm{H}), 4.27(\mathrm{~m}, 2 \mathrm{H}), 4.34(\mathrm{t}, J=7.9,2 \mathrm{H}), 4.56(\mathrm{~s}, 2 \mathrm{H}), 8.86(\mathrm{~s}, 1 \mathrm{H})$, $9.61(\mathrm{~s}, 1 \mathrm{H}), 9.78(\mathrm{~s}, 1 \mathrm{H}) ;{ }^{13} \mathrm{C}$ NMR $\left(500 \mathrm{MHz}, \mathrm{CDCl}_{3}\right) \delta 11.5,11.9,12.2,14.4,15.6,21.8,22.1$, 22.9, 29.6, 29.92, 30.0, 30.1, 30.8, 31.8, 32.2, 33.4, 34.6, 36.9, 37.4, 45.9, 51.2, 51.9, 52.0, 91.1, $97.5,98.9,111.5,128.0,129.6,131.3,133.5,135.3,136.1,137.5,137.9,138.1,138.8,149.3$, 150.4, 162.3, 171.8, 173.7, 174.2; UV $\left(\mathrm{CHCl}_{3}\right): \lambda_{\max }\left(\varepsilon \mathrm{L} \mathrm{mol}^{-1}, \mathrm{~cm}^{-1}\right)=402(196000), 505$ (18400), 649 (53200) nm; HRMS (EI) Calcd for $\mathrm{C}_{44} \mathrm{H}_{60} \mathrm{~N}_{4} \mathrm{O}_{4}$ : 708.4615; found: 708.4623 . 
4-[10-Decyl-18-(3-methoxycarbonyl-propyl)-3,7,8,13,13,17-hexamethyl-12,13,22,24-tetrahydro-porphin-2-yl]-butyric acid methyl ester (8ec). Following the procedure outlined for 8aa, reaction of $6 \mathrm{e}(5.8 \mathrm{mg}, 15 \mu \mathrm{mol})$ with $7 \mathbf{c}(6.7 \mathrm{mg}, 15 \mu \mathrm{mol})$ in $\mathrm{CH}_{2} \mathrm{Cl}_{2}(1.3 \mathrm{~mL})$ and TFA $(67 \mu \mathrm{L})$ gave 8ec $(7.7 \mathrm{mg}, 72 \%)$ as a green film after chromatography (silica gel, EtOAc:hexanes $=1: 4$, $\left.1 \% \mathrm{NEt}_{3}\right) . \mathrm{R}_{f}(1: 4 \mathrm{EtOAc} /$ hexanes $)$ 0.24; IR(thin film) $1735,1603,1437,1158 \mathrm{~cm}^{-1} ; \mathrm{UV}\left(\mathrm{CH}_{2} \mathrm{Cl}_{2}\right)$ : $\lambda_{\max }\left(\varepsilon \mathrm{L} \mathrm{mol}^{-1} \mathrm{~cm}^{-1}\right)=648(48400), 595,502,399 \mathrm{~nm} ;{ }^{1} \mathrm{H}$ NMR $\left(500 \mathrm{MHz}, \mathrm{CDCl}_{3}\right) \delta-2.47$ (br s, $1 \mathrm{H}),-2.00$ (br s, 1H), $0.92(\mathrm{t}, J=6.9,3 \mathrm{H}), 1.27-1.47$ (br m, 10H), $1.55(\mathrm{~m}, 2 \mathrm{H}), 1.81(\mathrm{~m}, 2 \mathrm{H}), 2.06$ $(\mathrm{s}, 6 \mathrm{H}), 2.11(\mathrm{~m}, 2 \mathrm{H}), 2.54(\mathrm{~m}, 4 \mathrm{H}), 2.67(\mathrm{~m}, 4 \mathrm{H}), 3.43(\mathrm{~s}, 3 \mathrm{H}), 3.47(\mathrm{~s}, 3 \mathrm{H}), 3.52(\mathrm{~s}, 3 \mathrm{H}), 3.56(\mathrm{~s}$, $3 \mathrm{H}), 3.70(\mathrm{~s}, 3 \mathrm{H}), 3.71(\mathrm{~s}, 3 \mathrm{H}), 3.90(\mathrm{t}, J=7.5,2 \mathrm{H}), 4.04(\mathrm{t}, J=7.5,2 \mathrm{H}), 4.29(\mathrm{~m}, 2 \mathrm{H}), 4.55(\mathrm{~s}$, 2H), $8.81(\mathrm{~s}, 1 \mathrm{H}), 9.65(\mathrm{~s}, 1 \mathrm{H}), 9.77(\mathrm{~s}, 1 \mathrm{H}) ;{ }^{13} \mathrm{C} \mathrm{NMR}\left(500 \mathrm{MHz}, \mathrm{CDCl}_{3}\right) \delta 11.6,12.0,12.3,14.4$, $15.7,23.0,25.5,25.9,27.8,28.3,29.7,30.0,30.05,30.1,30.8,31.9,32.2,33.5,33.8,33.9,34.6$, $45.9,51.3,51.7,51.8,90.9,98.0,98.9,111.4,127.8,129.4,131.2,134.1,135.9,136.5,137.6$, 137.7, 138.0, 139.8, 149.4, 151.2, 162.0, 171.7, 174.4, 174.7. HRMS (EI) Calcd for $\mathrm{C}_{46} \mathrm{H}_{64} \mathrm{~N}_{4} \mathrm{O}_{4}$ : 736.4928; found: 736.4919 .

5-[10-Decyl-18-(4-methoxycarbonyl-butyl)-3,7,8,13,13,17-hexamethyl-12,13,22,24-tetrahydro-porphin-2-yl]-pentanoic acid methyl ester (8ed). Following the procedure outlined for 8aa, reaction of $6 \mathbf{e}(5.8 \mathrm{mg}, 15 \mu \mathrm{mol})$ with $7 \mathbf{d}(7.2 \mathrm{mg}, 15 \mu \mathrm{mol})$ in $\mathrm{CH}_{2} \mathrm{Cl}_{2}(1.3 \mathrm{~mL})$ and TFA (67 $\mu \mathrm{L})$ gave 8ed $(9.5 \mathrm{mg}, 85 \%)$ as a green film after chromatography (silica gel, EtOAc:hexanes $=$ $\left.1: 4,1 \% \mathrm{NEt}_{3}\right) . \mathrm{R}_{f}(1: 4 \mathrm{EtOAc} /$ hexanes $)$ 0.25; IR(thin film) 1738, 1603, 1436, $1149 \mathrm{~cm}^{-1}$; UV $\left(\mathrm{CH}_{2} \mathrm{Cl}_{2}\right): \lambda_{\max }\left(\varepsilon \mathrm{L} \mathrm{mol}^{-1} \mathrm{~cm}^{-1}\right)=648(30300), 501,399 \mathrm{~nm} ;{ }^{1} \mathrm{H}$ NMR $\left(500 \mathrm{MHz}, \mathrm{CDCl}_{3}\right) \delta-2.47$ (br s, 1H), -2.01 (br s, 1H), $0.92(\mathrm{t}, J=6.9,3 \mathrm{H}), 1.27-1.45$ (br m, $10 \mathrm{H}), 1.55(\mathrm{~m}, 2 \mathrm{H}), 1.81(\mathrm{~m}$, 2H), 1.98-2.06 (overlapping singlet and multiplet, 10H), $2.11(\mathrm{~m}, 2 \mathrm{H}), 2.24(\mathrm{~m}, 4 \mathrm{H}), 2.47(\mathrm{t}, J=$ 7.5, 4H), $3.43(\mathrm{~s}, 3 \mathrm{H}), 3.46(\mathrm{~s}, 3 \mathrm{H}), 3.52(\mathrm{~s}, 3 \mathrm{H}), 3.56(\mathrm{~s}, 3 \mathrm{H}), 3.64$ (overlapping singlets, $6 \mathrm{H})$, $3.86(\mathrm{t}, J=7.5,2 \mathrm{H}), 3.99(\mathrm{t}, J=7.5,2 \mathrm{H}), 4.28(\mathrm{~m}, 2 \mathrm{H}), 4.55(\mathrm{~s}, 2 \mathrm{H}), 8.81(\mathrm{~s}, 1 \mathrm{H}), 9.52(\mathrm{~s}, 1 \mathrm{H})$, $9.77(\mathrm{~s}, 1 \mathrm{H}) ;{ }^{13} \mathrm{C} \mathrm{NMR}\left(500 \mathrm{MHz}, \mathrm{CDCl}_{3}\right) \delta 11.6,12.1,12.3,14.4,15.6,23.0,25.4,25.5,26.1$, 26.4, 29.7, 30.0, 30.05, 30.1, 30.8, 31.9, 32.2, 32.4, 32.8, 33.5, 34.4, 34.5, 34.6, 45.9, 51.2, 51.7, 51.8, 90.9, 97.7, 98.9, 111.3, 127.7, 129.2, 131.2, 134.1, 135.9, 137.1, 137.4, 137.7, 137.9, 140.4, 149.4, 151.1, 161.9, 171.7, 174.3, 174.5. HRMS (EI) Calcd for $\mathrm{C}_{48} \mathrm{H}_{68} \mathrm{~N}_{4} \mathrm{O}_{4}$ : 764.5241; found: 764.5242 .

11-[10-Decyl-18-(10-methoxycarbonyl-decyl)-3,7,8,13,13,17-hexamethyl-12,13,22,24-tetrahydro-porphin-2-yl]-undecanoic acid methyl ester (8ee). Following the procedure outlined for 8aa, reaction of $6 \mathbf{e}(5.8 \mathrm{mg}, 15 \mu \mathrm{mol})$ with $7 \mathbf{e}(9.6 \mathrm{mg}, 15 \mu \mathrm{mol})$ in $\mathrm{CH}_{2} \mathrm{Cl}_{2}(1.3 \mathrm{~mL})$ and TFA (67 $\mu \mathrm{L})$ gave 8ee $(11 \mathrm{mg}, 77 \%)$ as a green film after chromatography (silica gel, EtOAc:hexanes = $\left.1: 4,1 \% \mathrm{NEt}_{3}\right) . \mathrm{R}_{f}$ (1:4 EtOAc/hexanes) 0.43 ; IR(thin film) 3302, 1740, 1603, 1457, $1157 \mathrm{~cm}^{-1}$; $\mathrm{UV}\left(\mathrm{CH}_{2} \mathrm{Cl}_{2}\right): \lambda_{\max }\left(\varepsilon \mathrm{L} \mathrm{mol}^{-1} \mathrm{~cm}^{-1}\right)=649$ (41900), 501, $399 \mathrm{~nm} ;{ }^{1} \mathrm{H} \mathrm{NMR}\left(500 \mathrm{MHz}, \mathrm{CDCl}_{3}\right) \delta-$ 2.45 (br s, 1H), -2.01 (br s, 1H), 0.92 (t, $J=7.0,3 \mathrm{H}), 1.27-1.47$ (overlapping multiplets, $30 \mathrm{H}$ ), $1.57(\mathrm{~m}, 6 \mathrm{H}), 1.66(\mathrm{~m}, 4 \mathrm{H}), 1.81(\mathrm{~m}, 2 \mathrm{H}), 2.06(\mathrm{~s}, 6 \mathrm{H}), 2.11(\mathrm{~m}, 2 \mathrm{H}), 2.18(\mathrm{~m}, 4 \mathrm{H}), 2.27(\mathrm{t}, J=$ 7.6, $4 \mathrm{H}), 3.43(\mathrm{~s}, 3 \mathrm{H}), 3.46(\mathrm{~s}, 3 \mathrm{H}), 3.52(\mathrm{~s}, 3 \mathrm{H}), 3.56(\mathrm{~s}, 3 \mathrm{H}), 3.65$ (overlapping singlets, $6 \mathrm{H})$, $3.82(\mathrm{t}, J=7.5,2 \mathrm{H}), 3.95(\mathrm{t}, J=7.5,2 \mathrm{H}), 4.28(\mathrm{~m}, 2 \mathrm{H}), 4.54(\mathrm{~s}, 2 \mathrm{H}), 8.81(\mathrm{~s}, 1 \mathrm{H}), 9.56(\mathrm{~s}, 1 \mathrm{H})$, $9.77(\mathrm{~s}, 1 \mathrm{H}) ;{ }^{13} \mathrm{C} \mathrm{NMR}\left(500 \mathrm{MHz}, \mathrm{CDCl}_{3}\right) \delta 11.6,12.0,12.3,14.4,15.6,23.0,25.2,26.4,26.7$, $29.39,29.41,29.52,29.54,29.7,29.78,29.79,29.89,29.95,29.97,30.02,30.06,30.08,30.11$, $30.15,30.2,30.8,31.8,32.2,33.0,33.50,33.53,34.3,34.6,45.9,51.2,51.7,90.8,98.0$, 98.9, $111.2,127.5,129.0,131.0,134.3,135.7,137.1,137.7,137.8,137.9,141.2,149.4,151.4,161.6$, 171.6, 174.6. HRMS (EI) Calcd for $\mathrm{C}_{60} \mathrm{H}_{92} \mathrm{~N}_{4} \mathrm{O}_{4}$ : 932.7119; found: 932.7099 . 


\section{References}

1. Jacobi, P.A.; Lanz, S.; Ghosh, I.; Leung, S.; Löwer, F; Pippin, D. Org. Lett. 2001, 3, 831.

2. Puzicha, G.; Shrout D.P.; Lightner, D.A. J. Heterocyclic Chem. 1990, 27, 2117-2123.

3. Jackson, A.H.; Pandey R.K.; Smith, K.M. J. Chem. Soc. Perkin Trans. 1 1987, 299-305.

4. Clezy, P.S.; Nichol, A.W. Aust. J. Chem. 1965, 11, 1835-1845.

5. Xie, H.; Lee, D.A.; Wallace, D.M.; Senge, M.O.; Smith, K.M. J. Org. Chem. 1996, 61, 8508-8517. 
wgo_1H_IV132
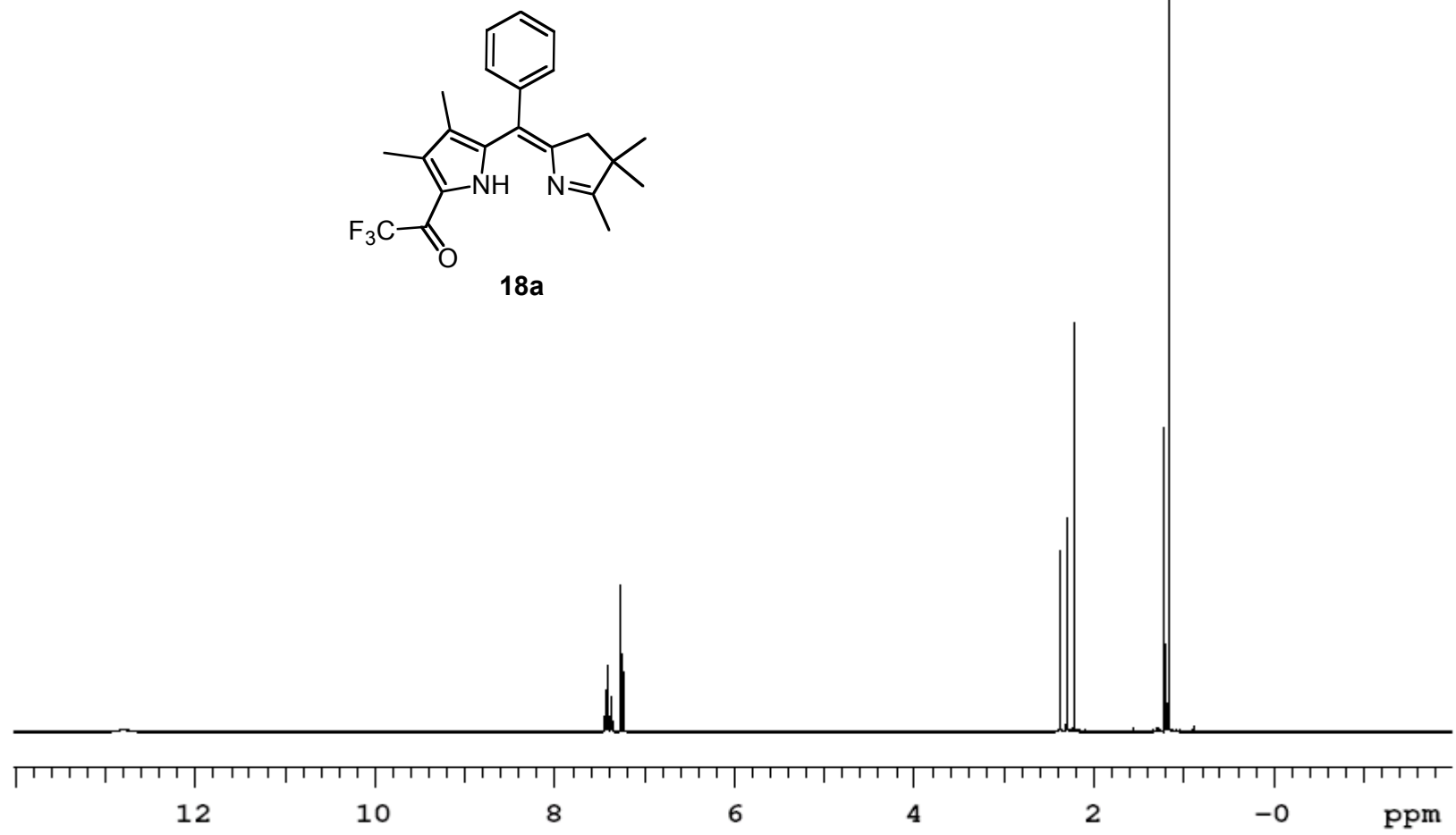

wgo $13 \mathrm{C} \quad$ IV132

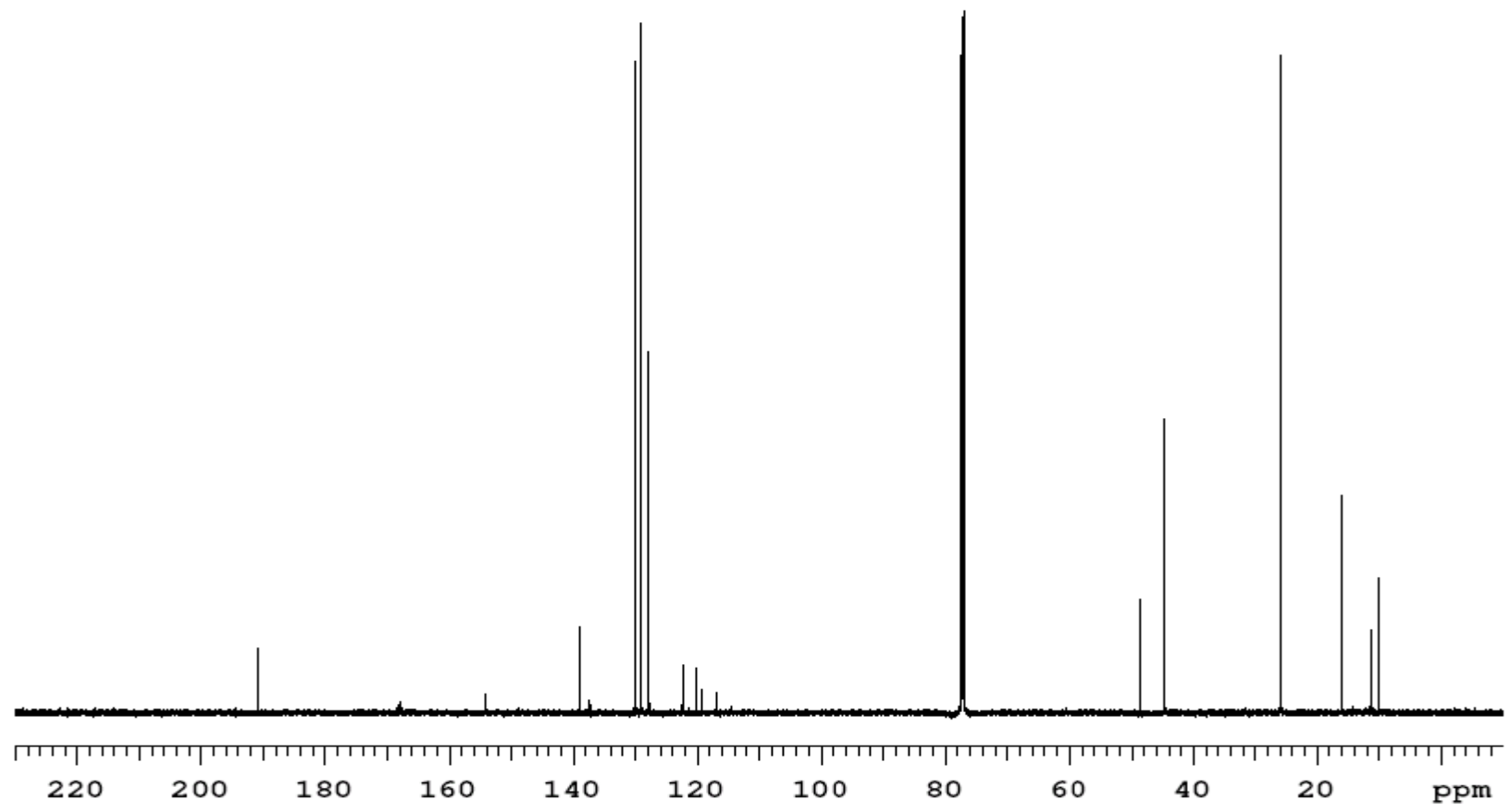


wr 5 p253
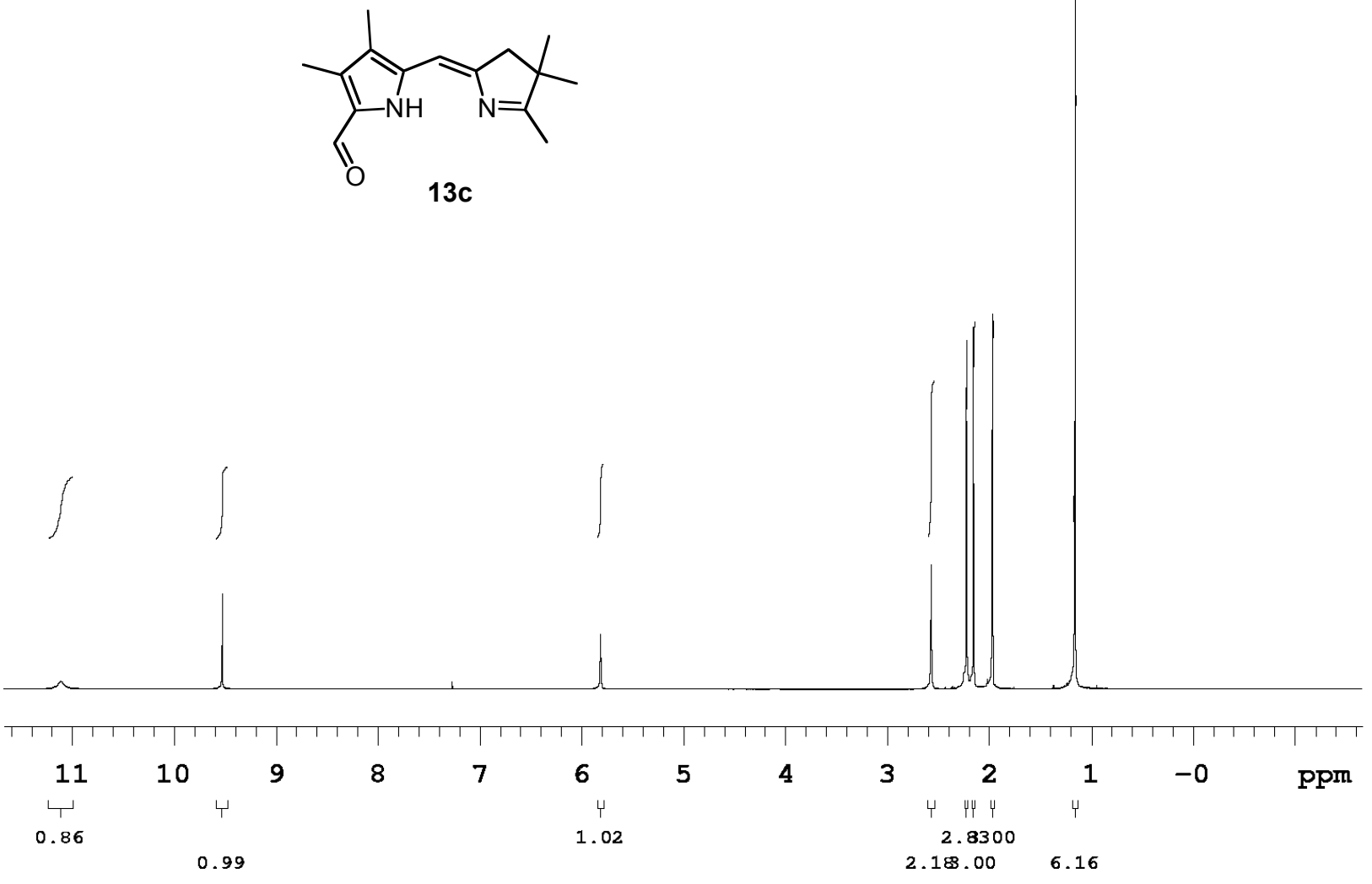

wr5p253
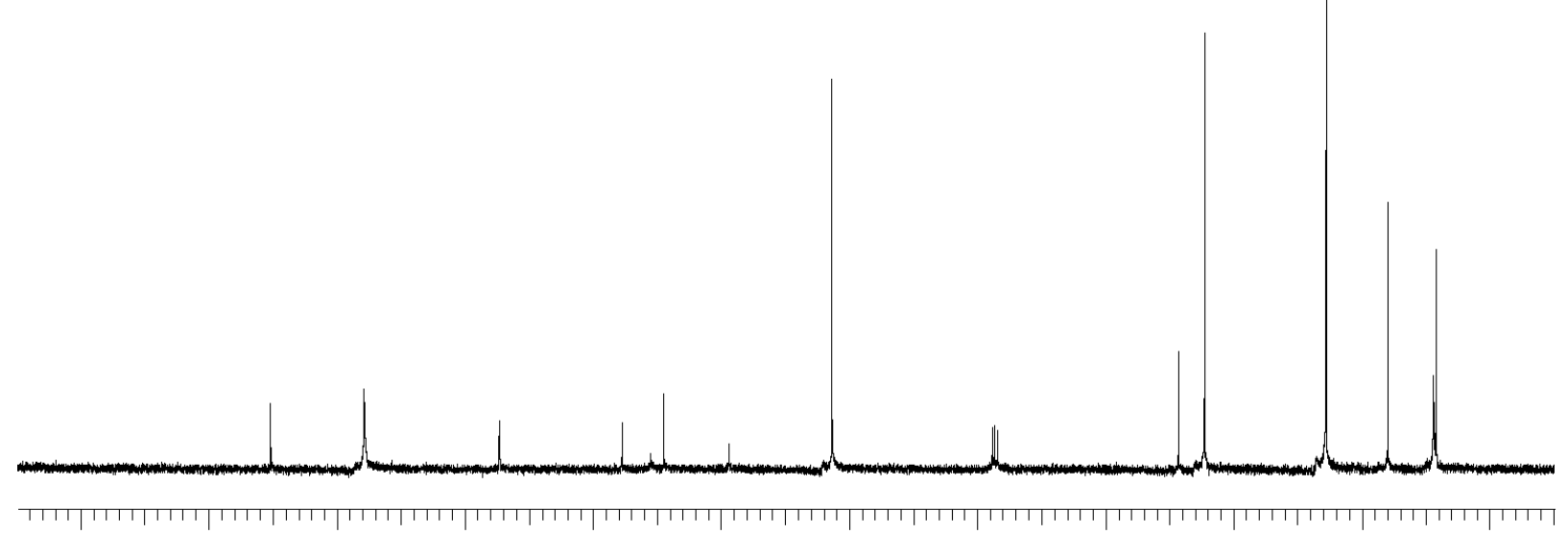
$\begin{array}{llllllllllll}220 & 200 & 180 & 160 & 140 & 120 & 100 & 80 & 60 & 40 & 20 & \text { Ppm }\end{array}$ 
wr5p158
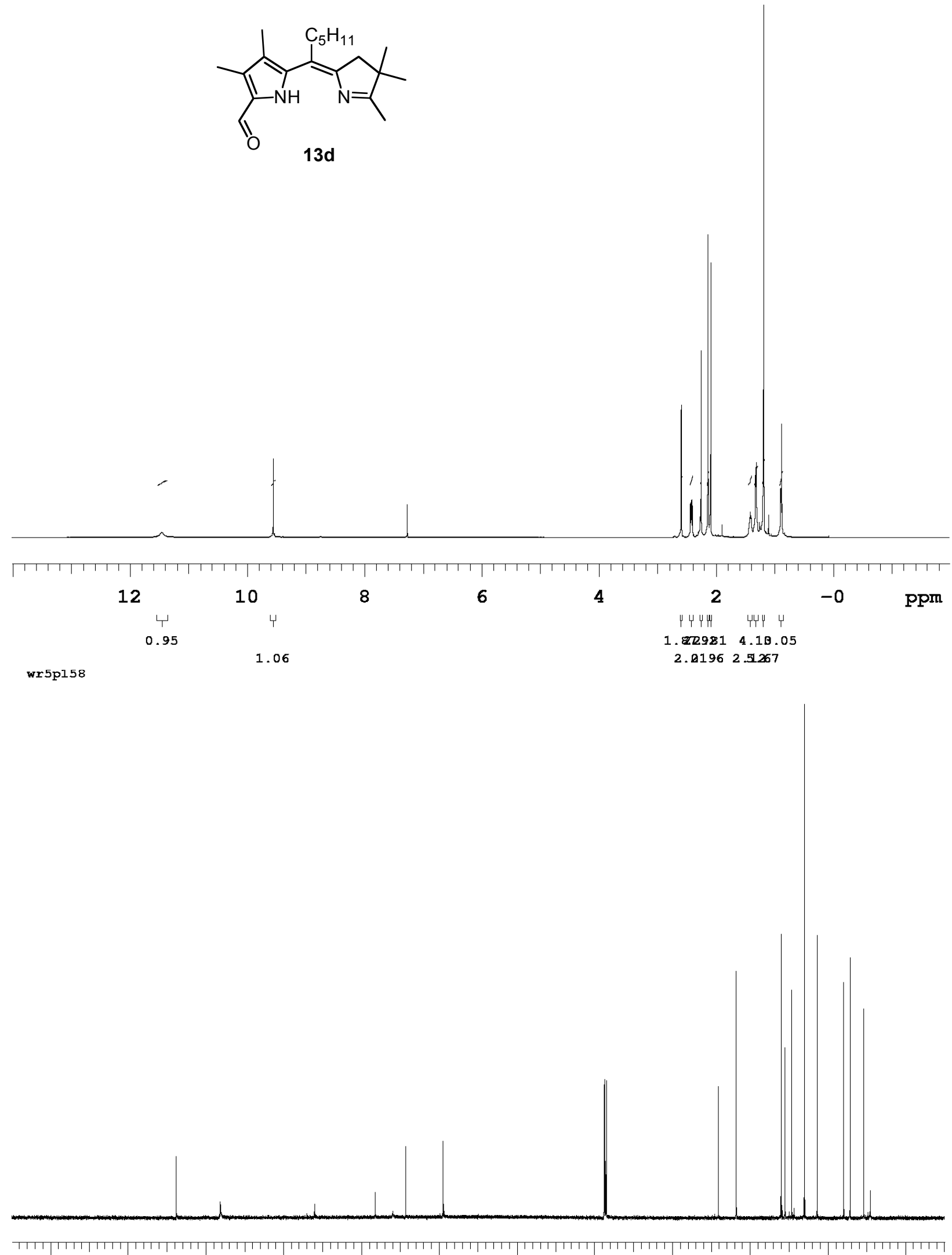

$\begin{array}{llllllllllll}220 & 200 & 180 & 160 & 140 & 120 & 100 & 80 & 60 & 40 & 20 & \text { Ppm }\end{array}$ 

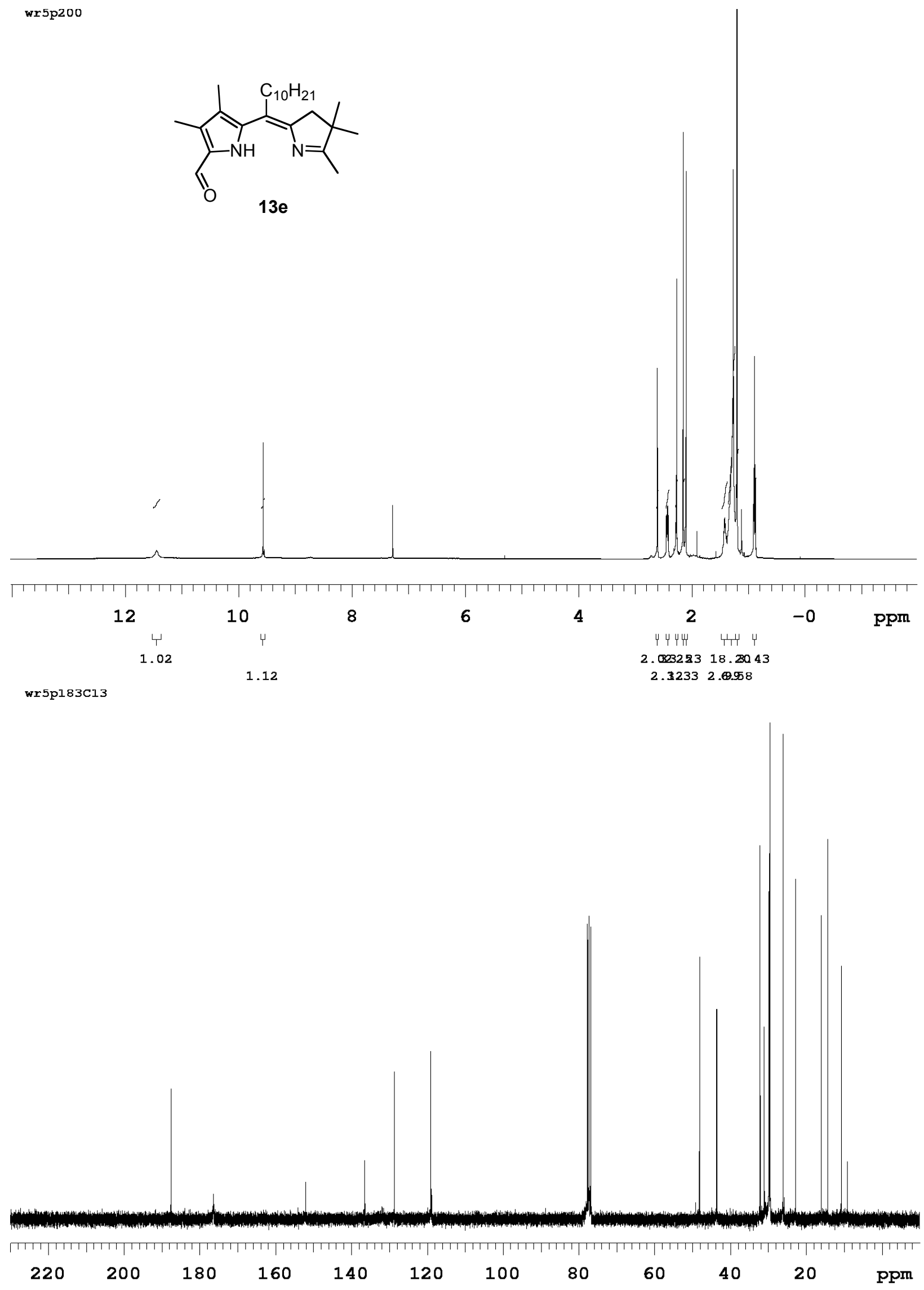
wgo_1H_III 257
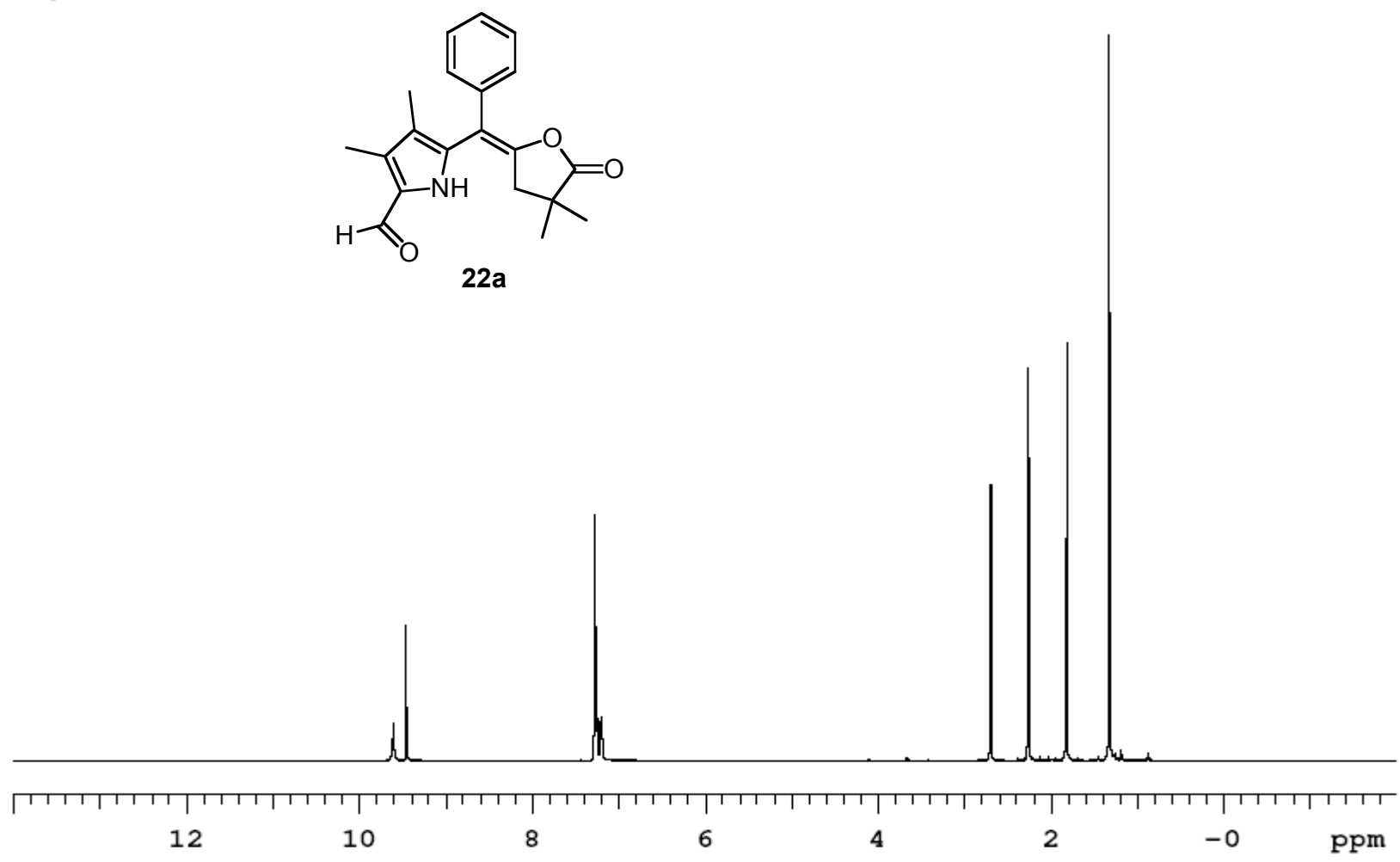

wgo_13C_III 257

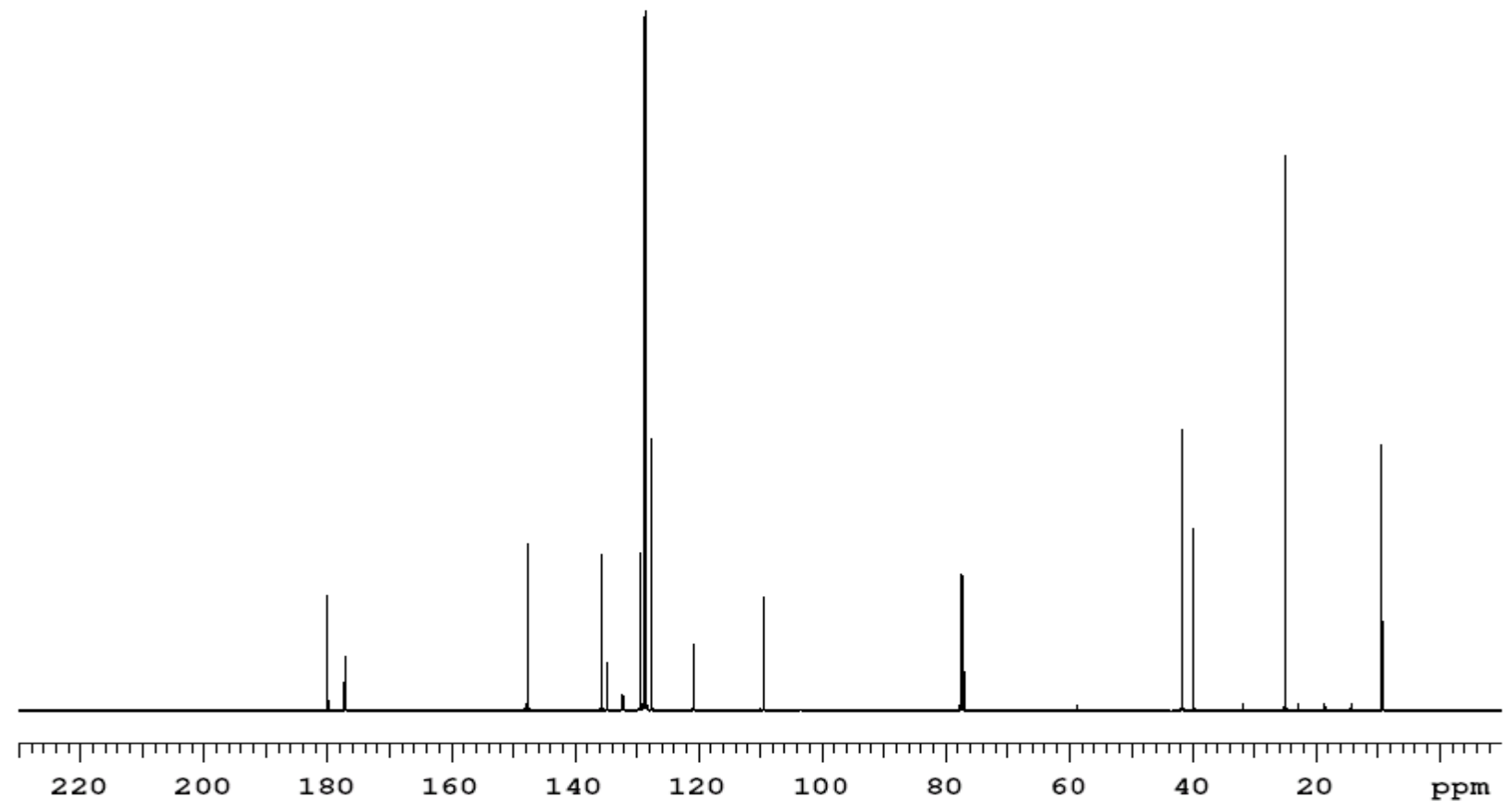


wgo_1H_III281
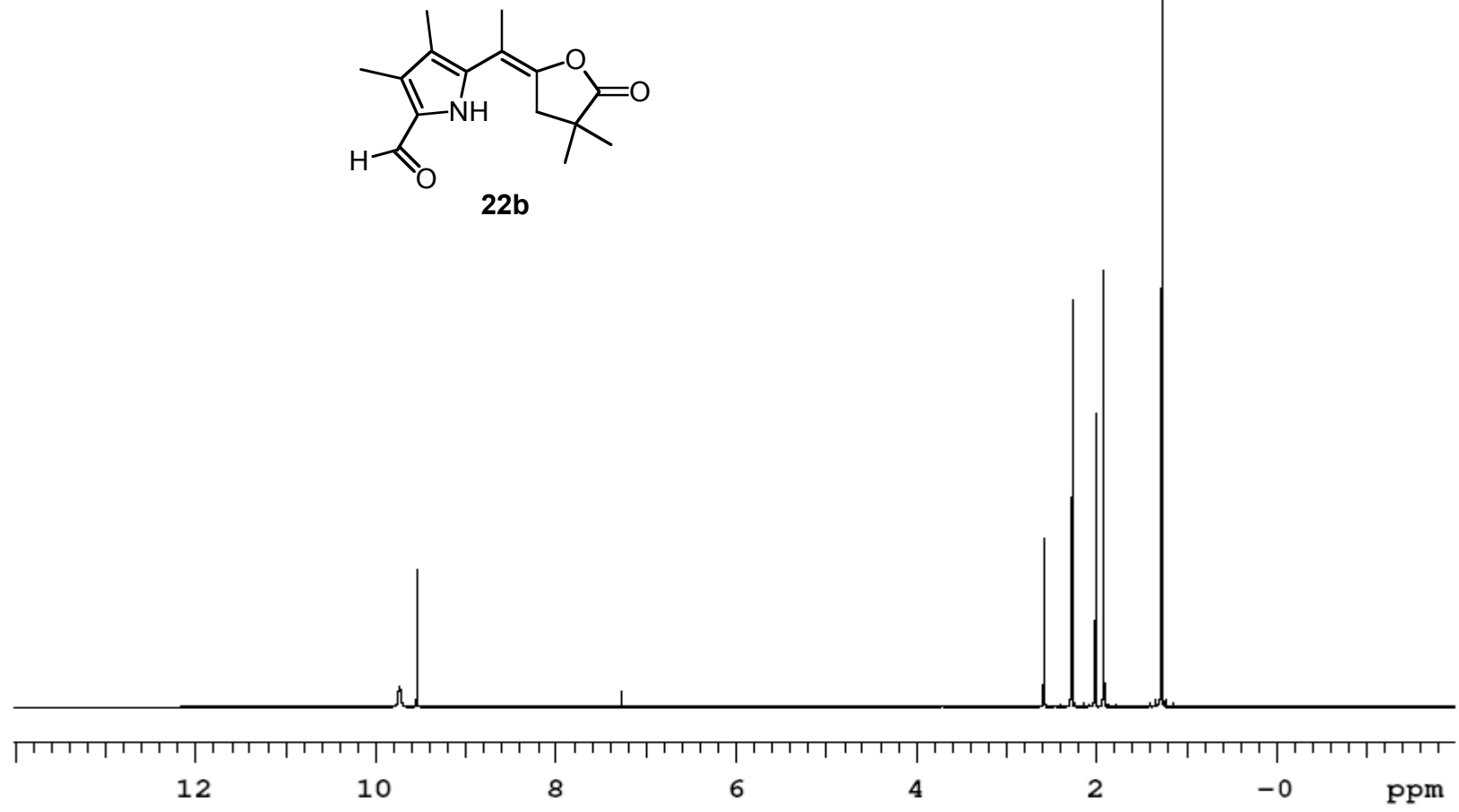

wgo_13C III281

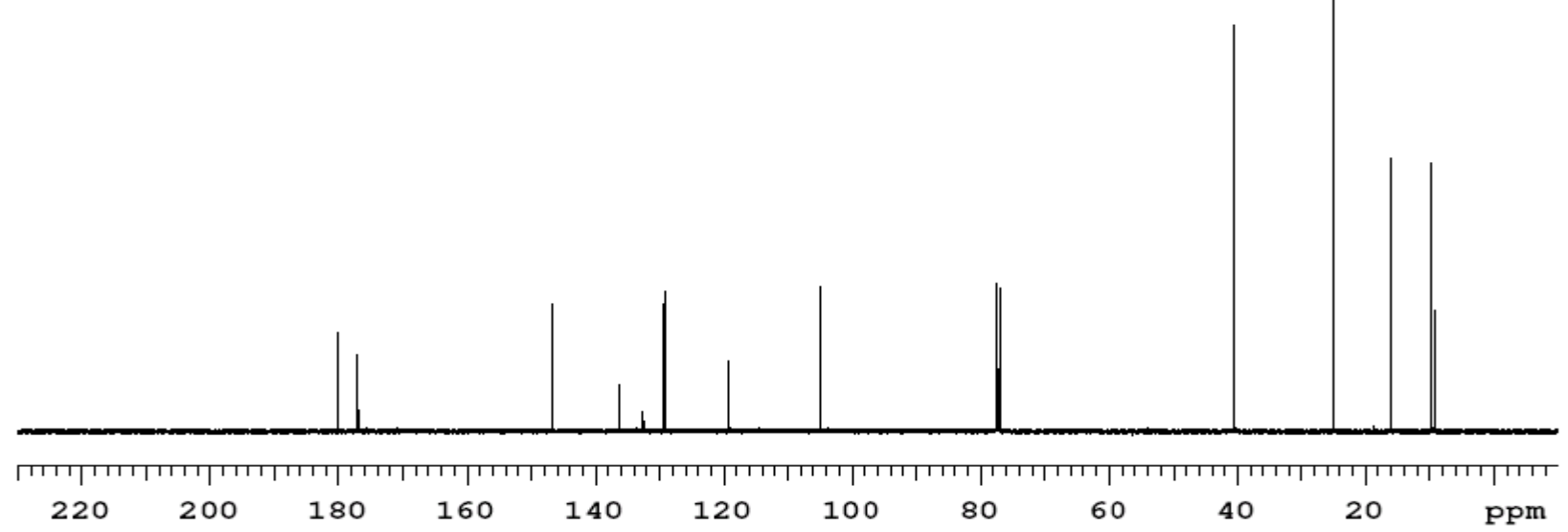


wr4p222
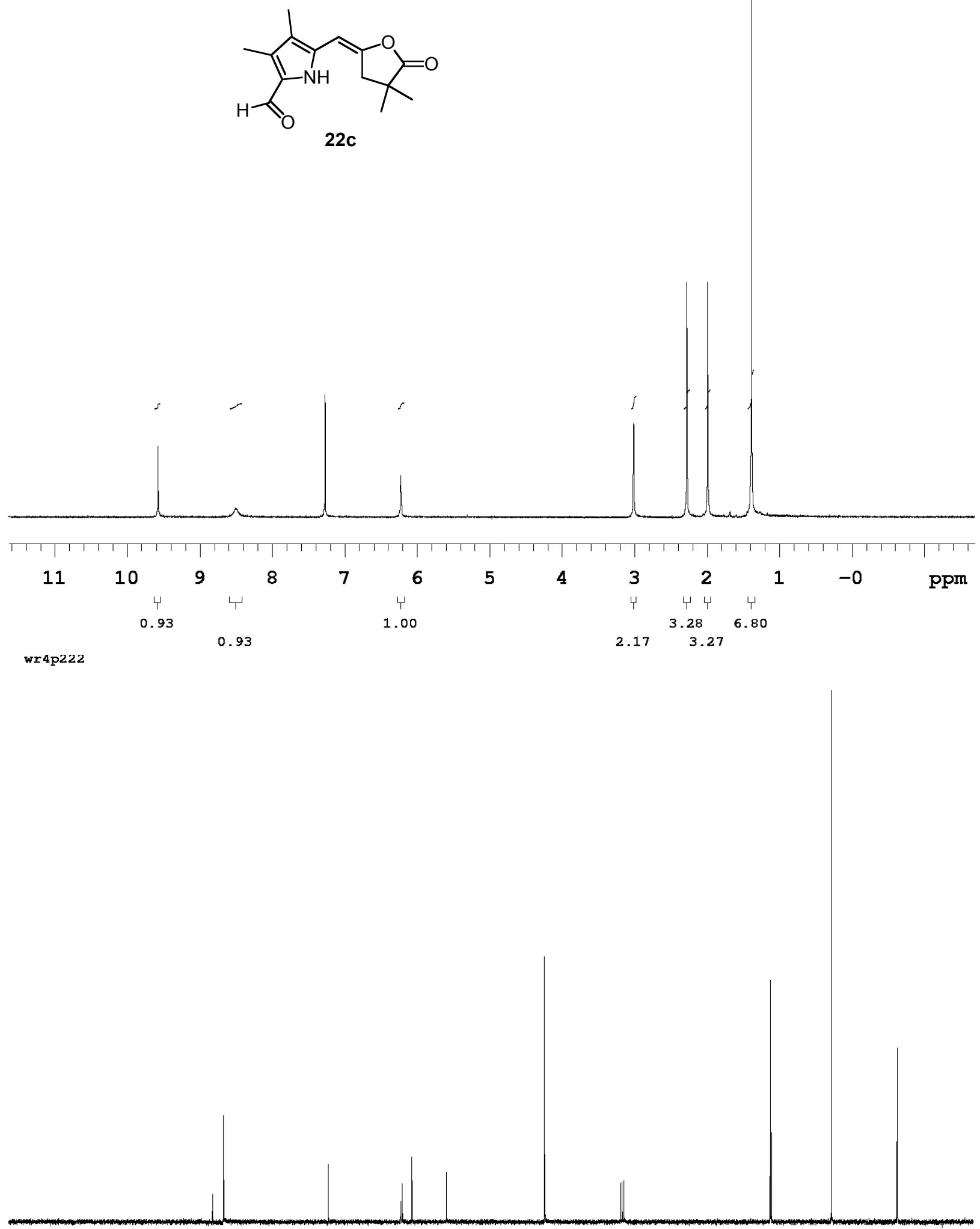

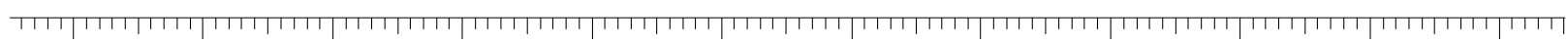
$\begin{array}{llllllllllll}220 & 200 & 180 & 160 & 140 & 120 & 100 & 80 & 60 & 40 & 20 & \text { Ppm }\end{array}$ 
wr6p226pp
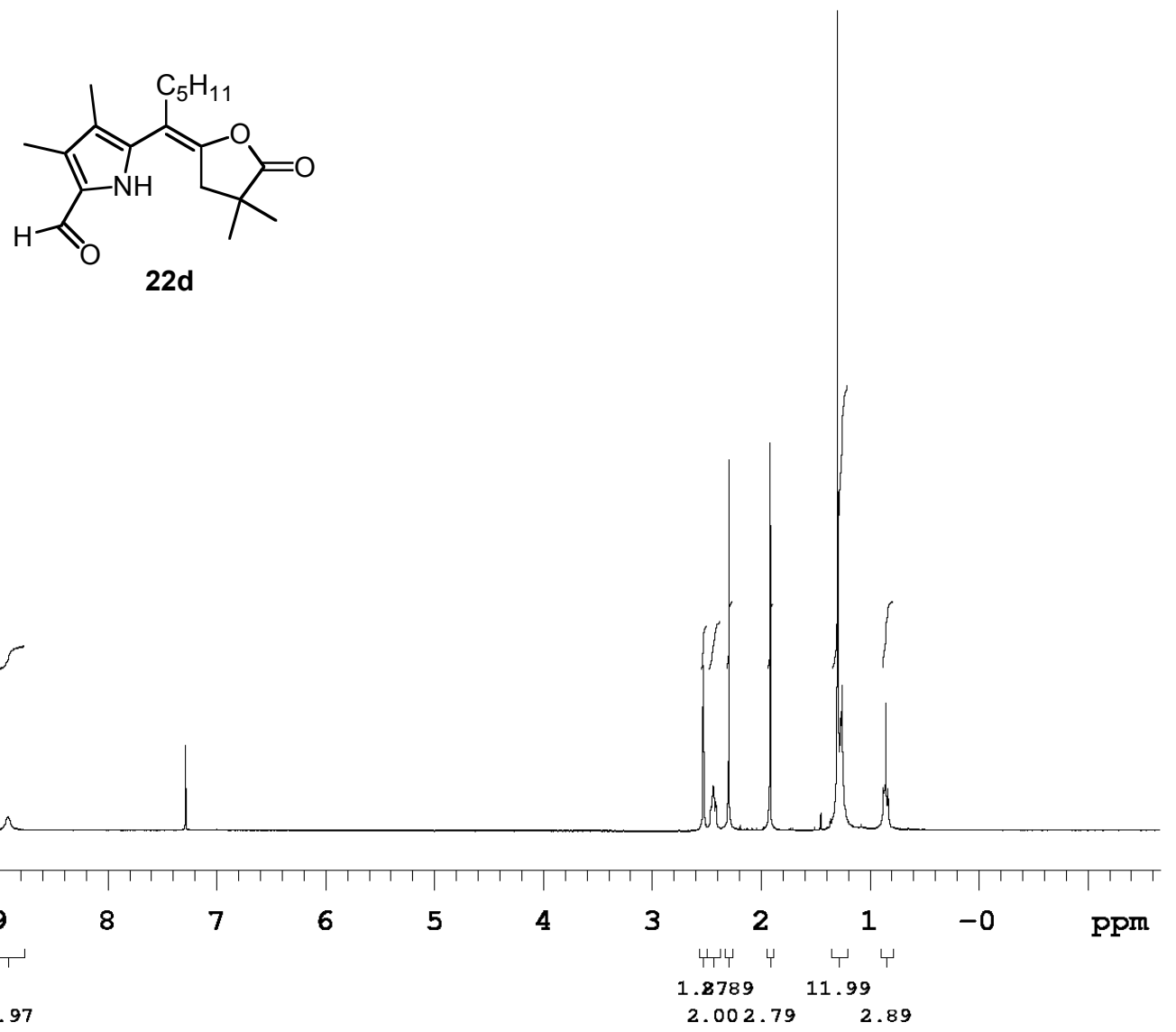

wr6p226C13
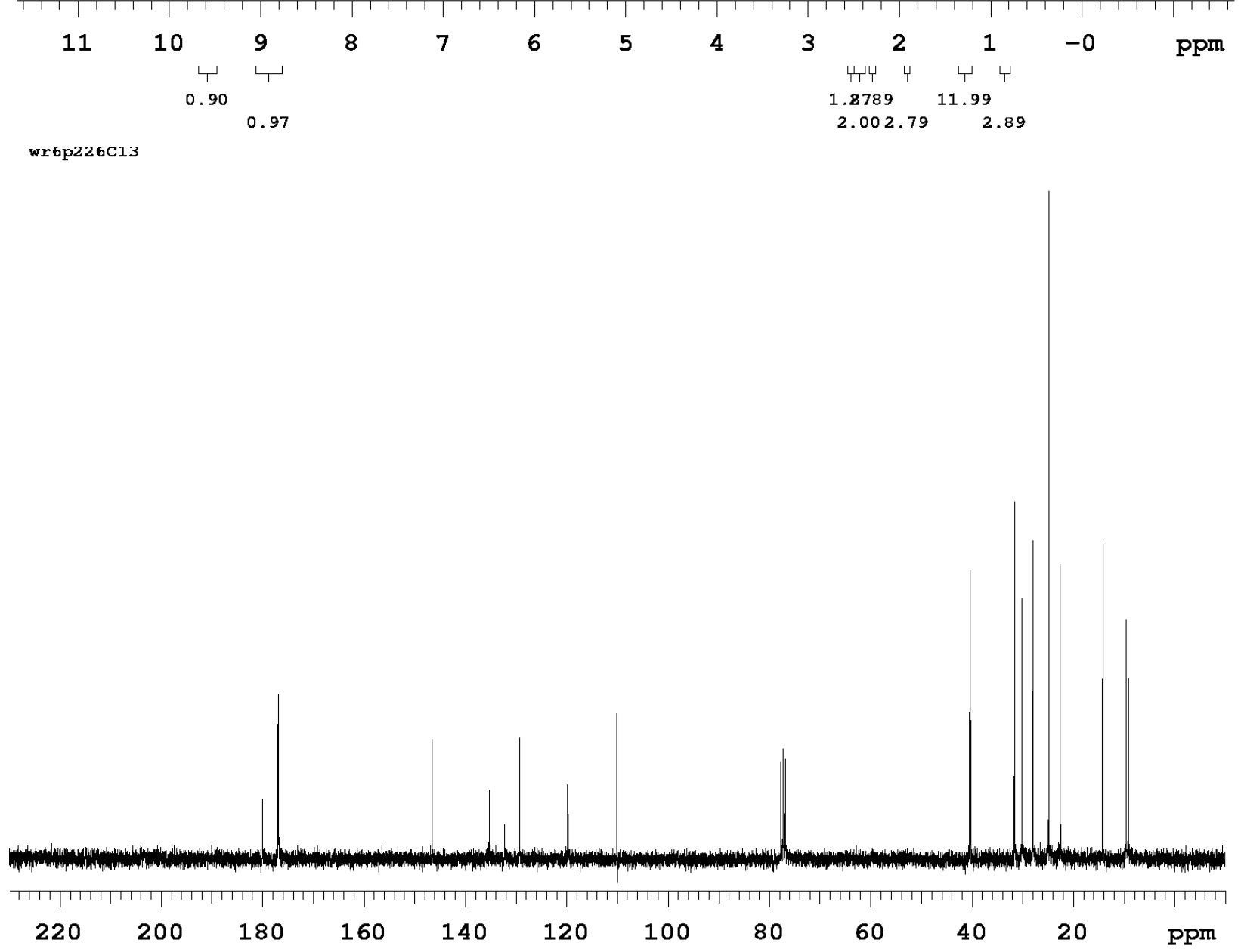
wgo_1H_III271-2
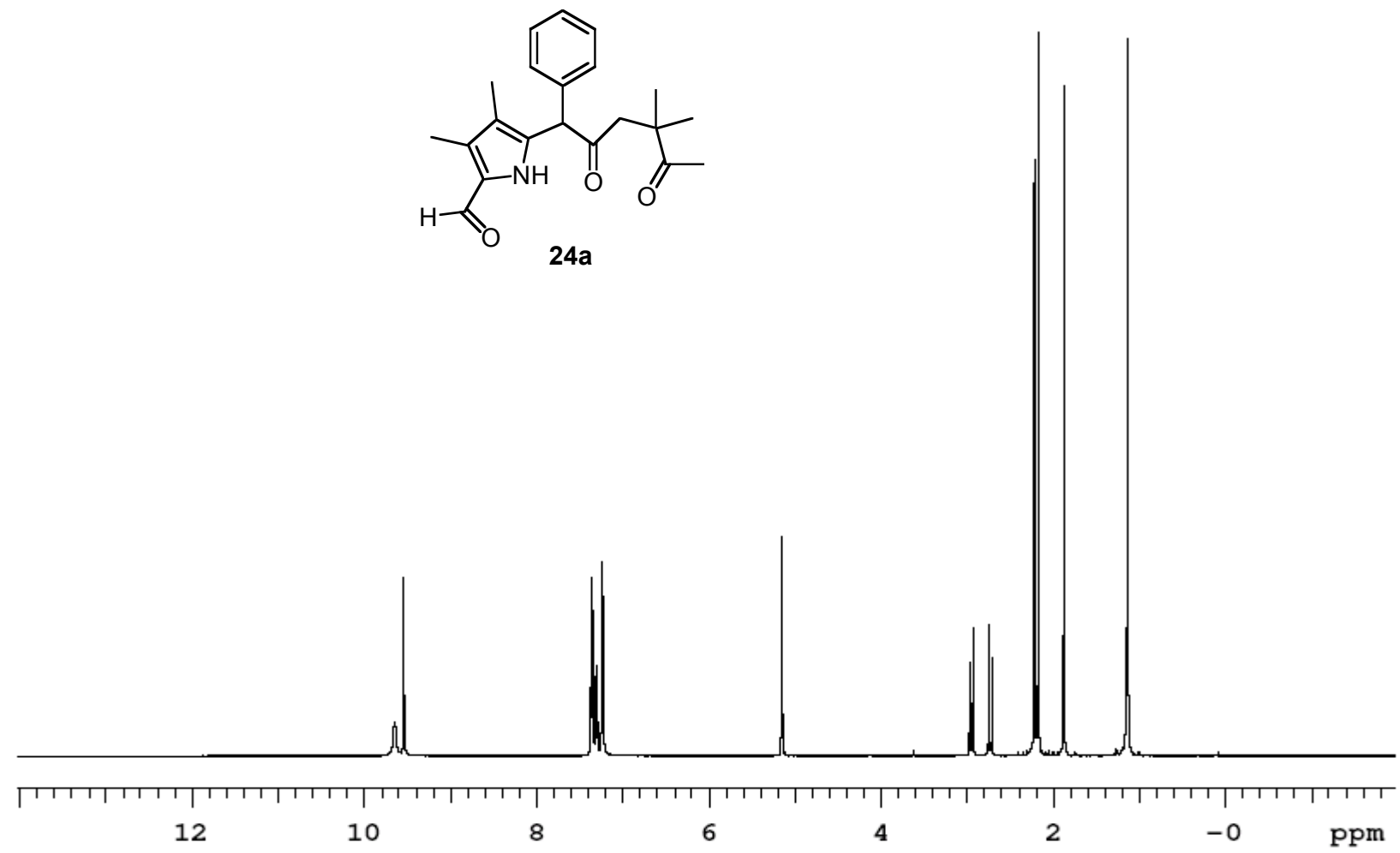

wgo 13C III 271-2

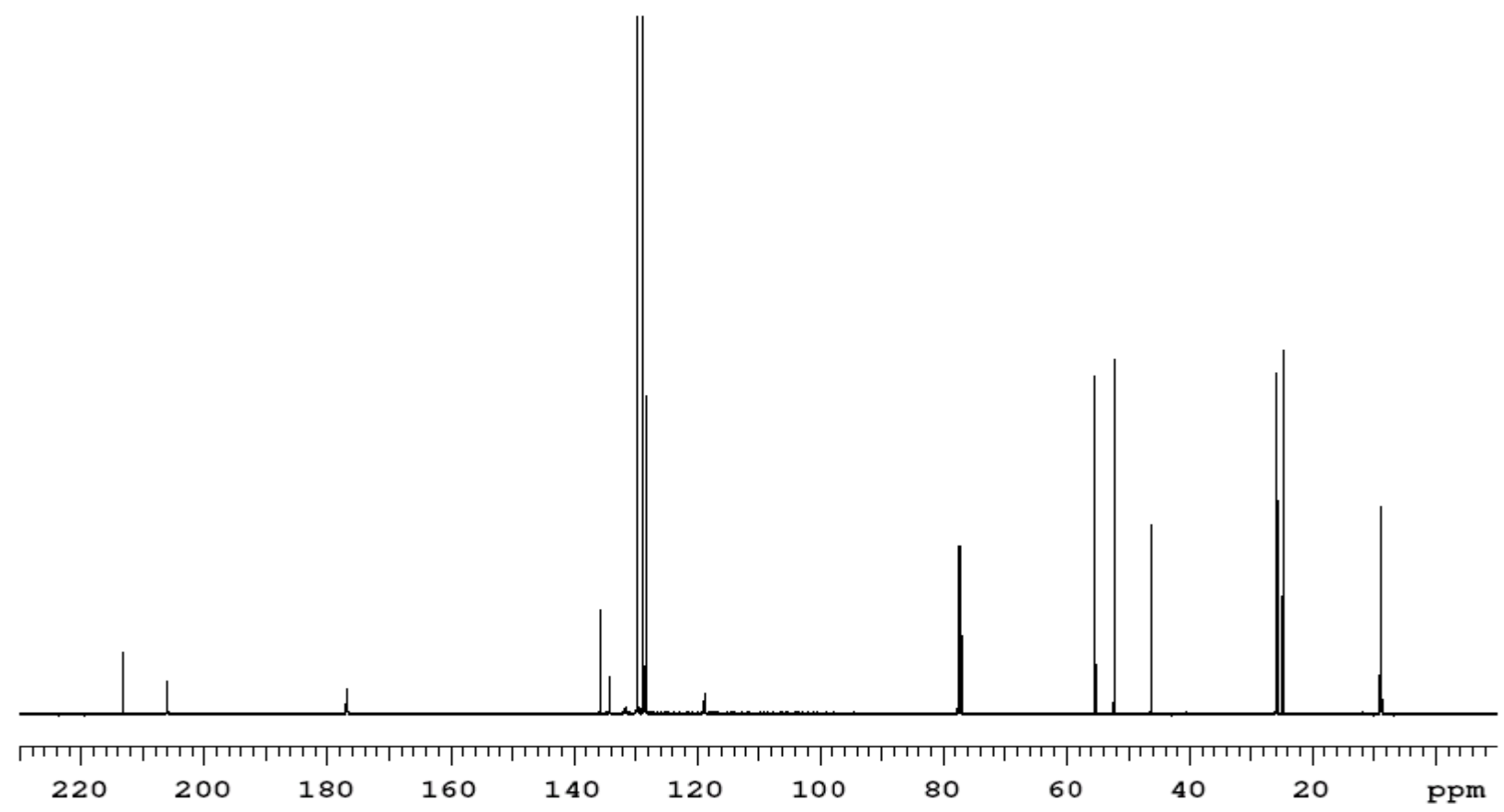


wr $6 \mathrm{p} 218$
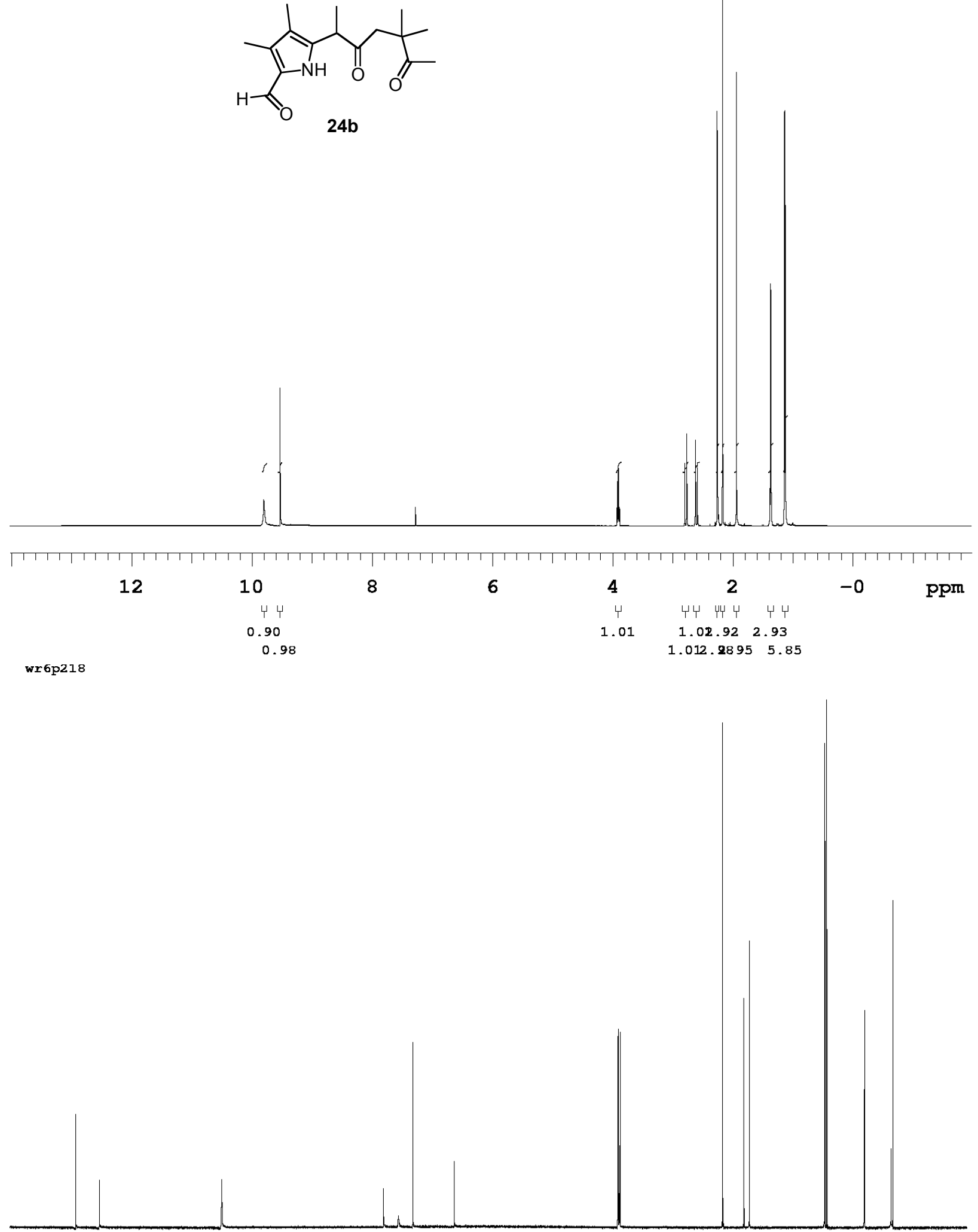

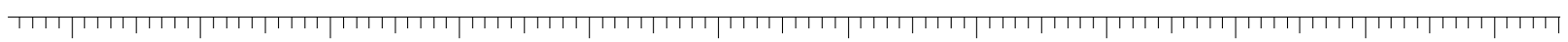
$\begin{array}{llllllllllll}220 & 200 & 180 & 160 & 140 & 120 & 100 & 80 & 60 & 40 & 20 & \text { Ppm }\end{array}$ 
wr5p039
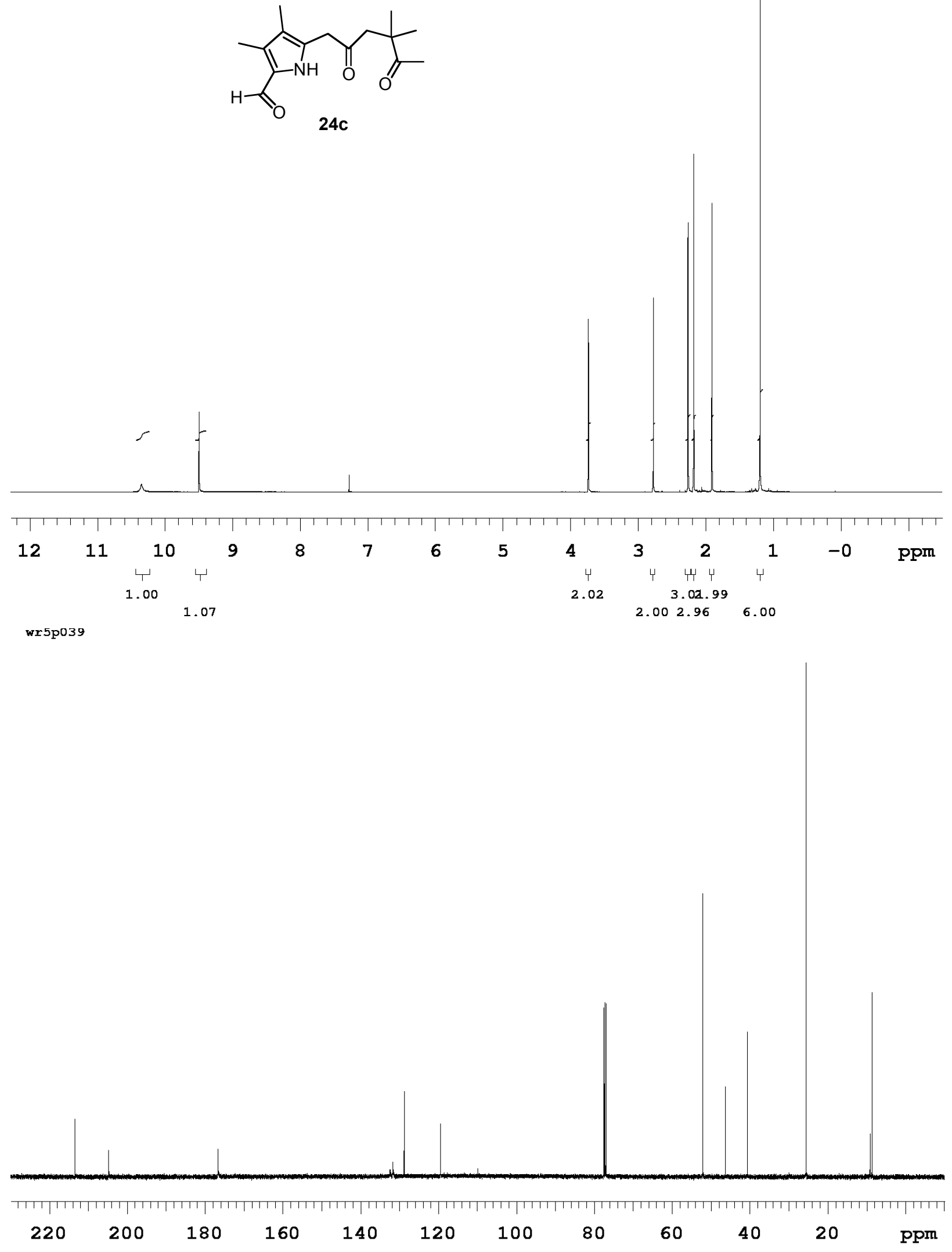
wr6p215cf10
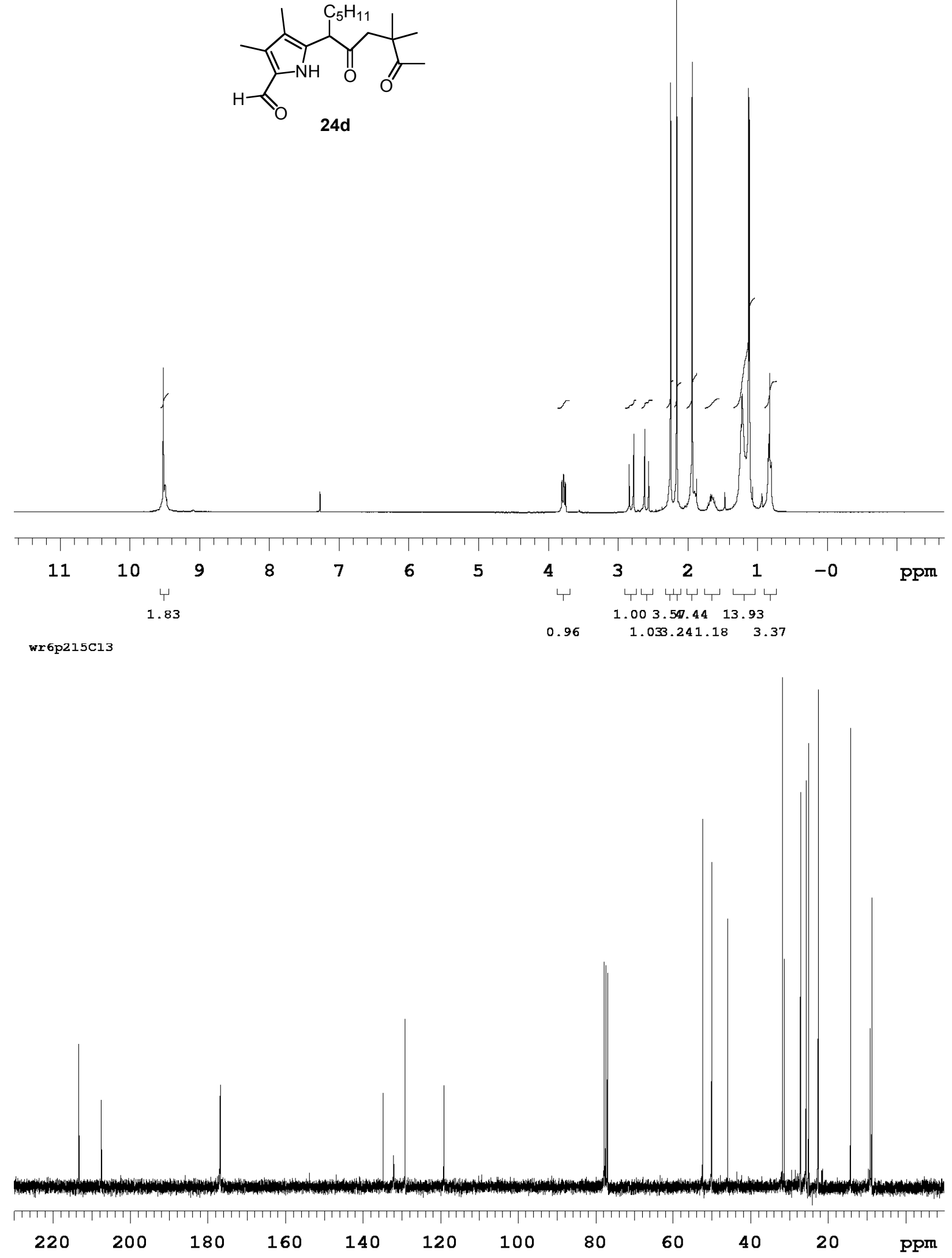
wr6p223cł12
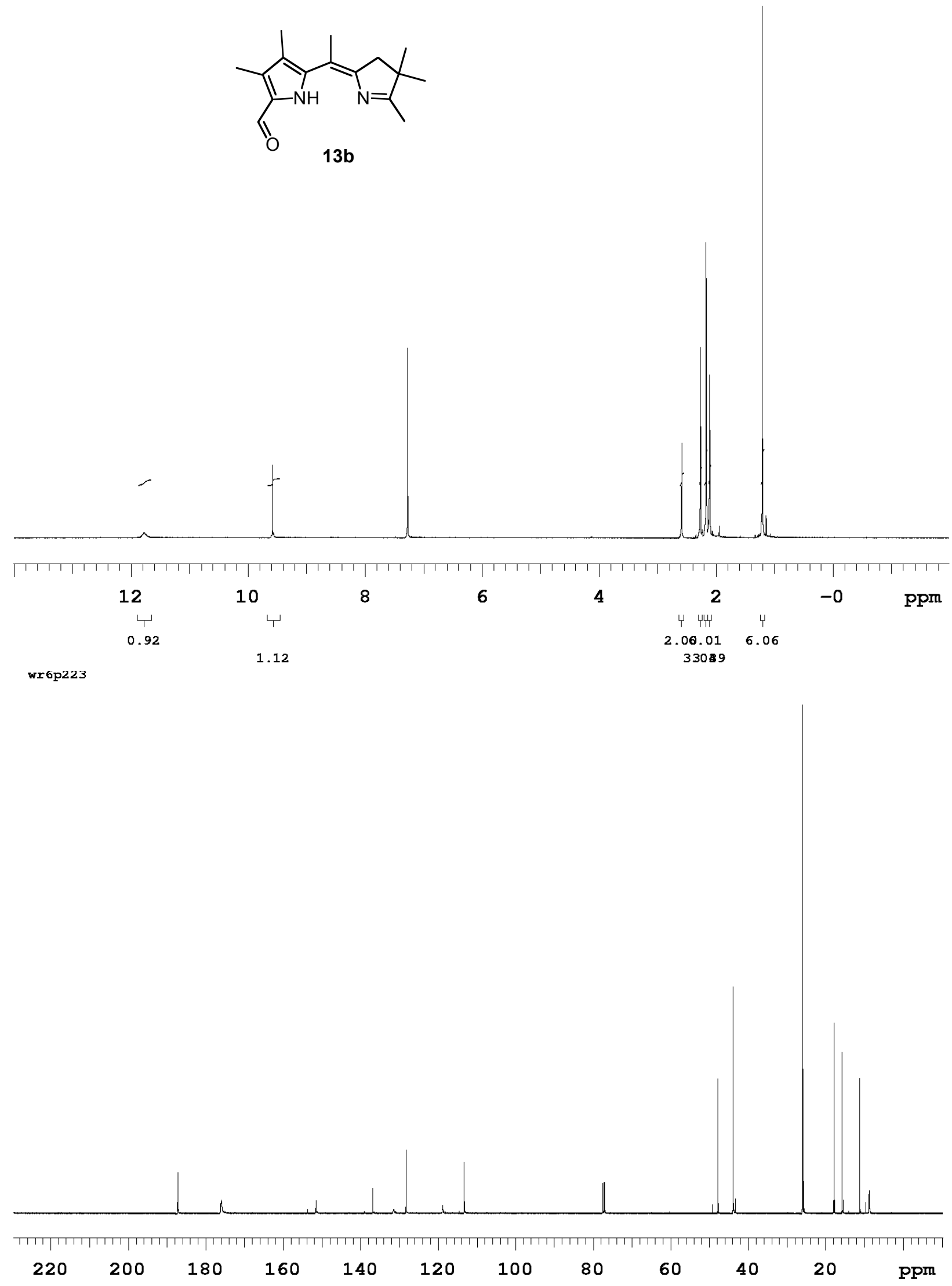
wr3p247c7

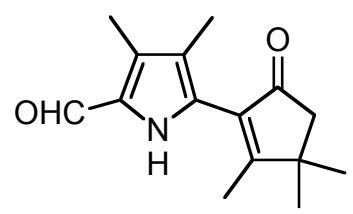

27c
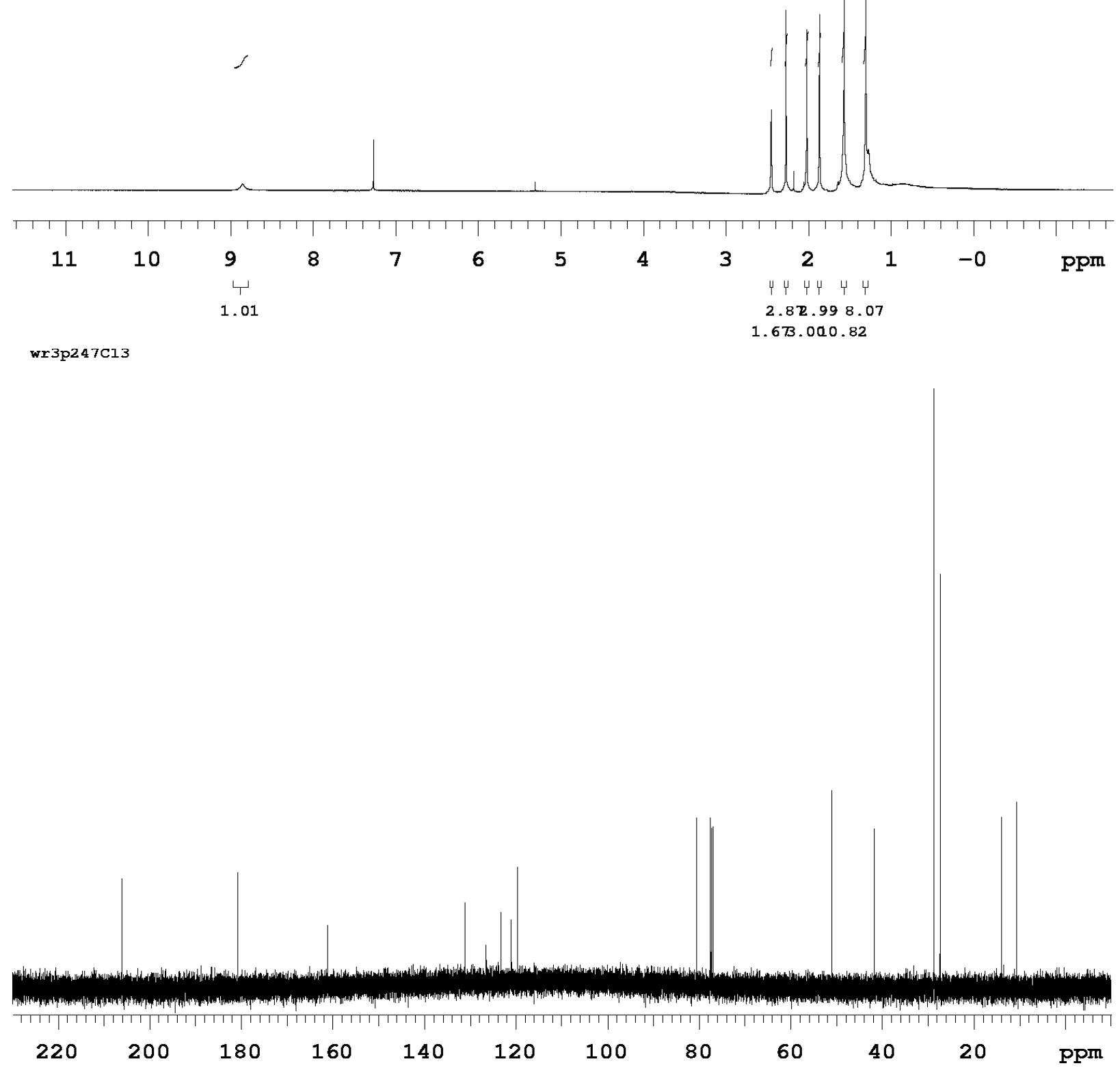
wgo_1H_TV135

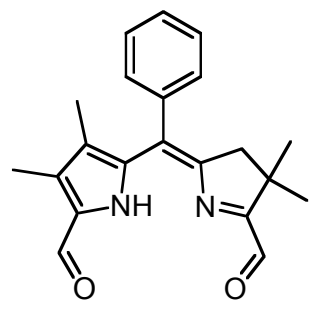

$6 a$
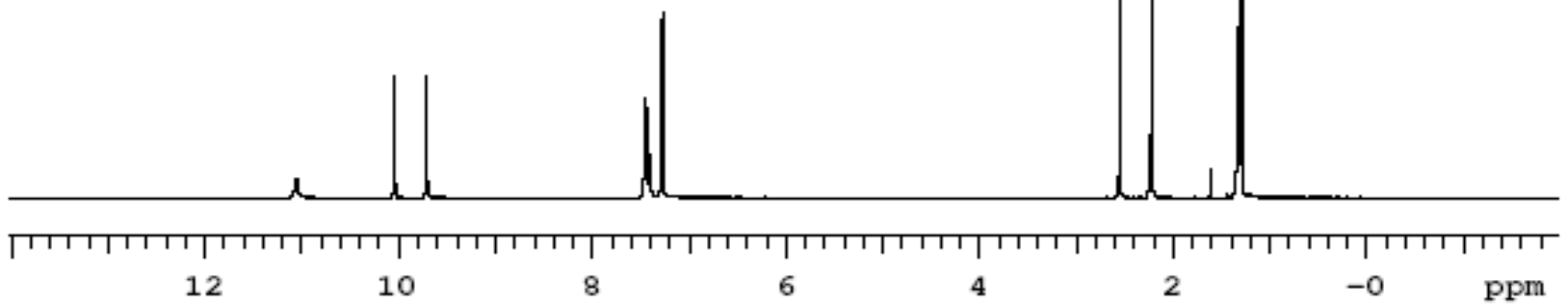

wgo_13C_IV135

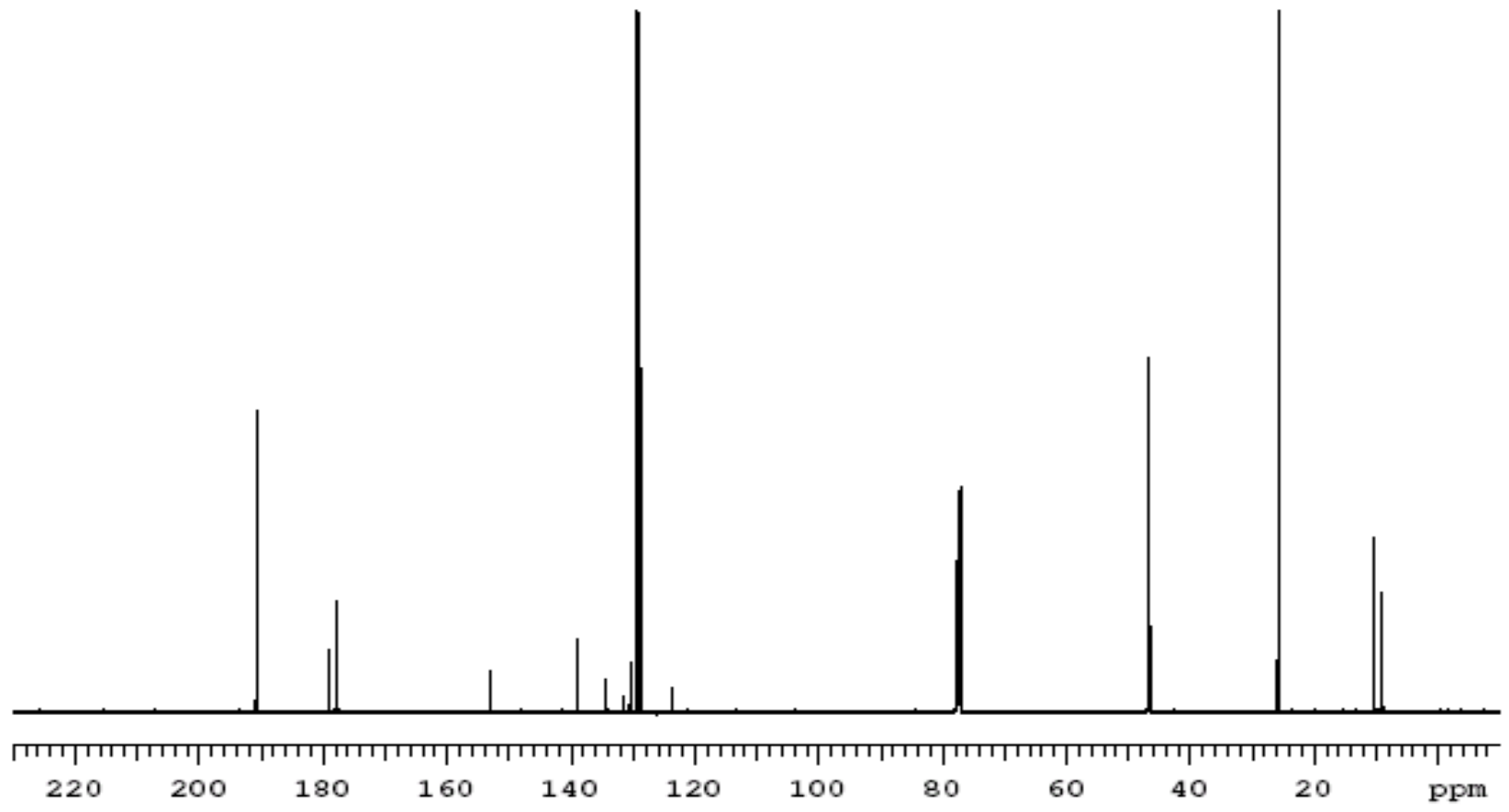

S34 
wr6p227cł11
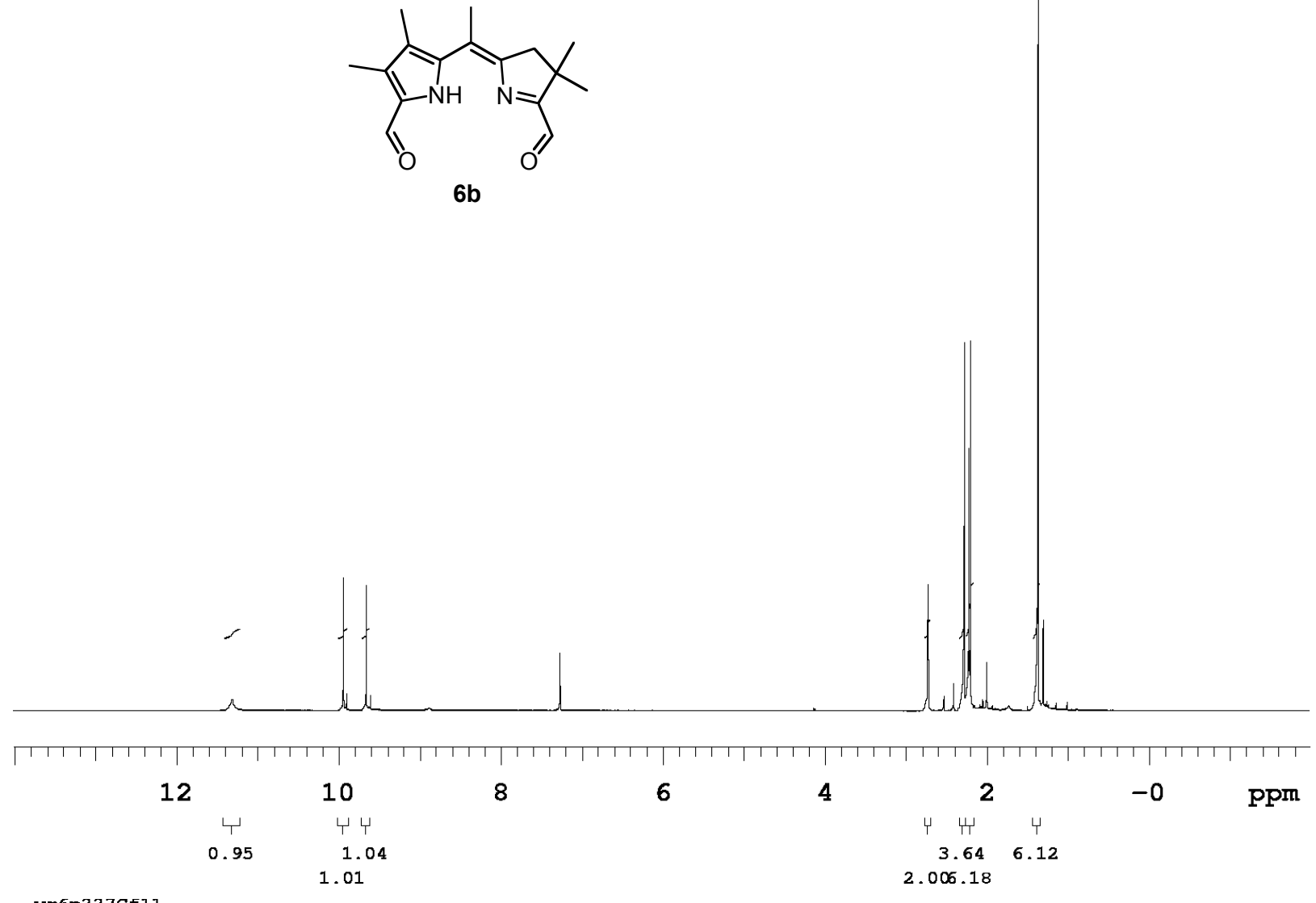

wr6p227CÆ11

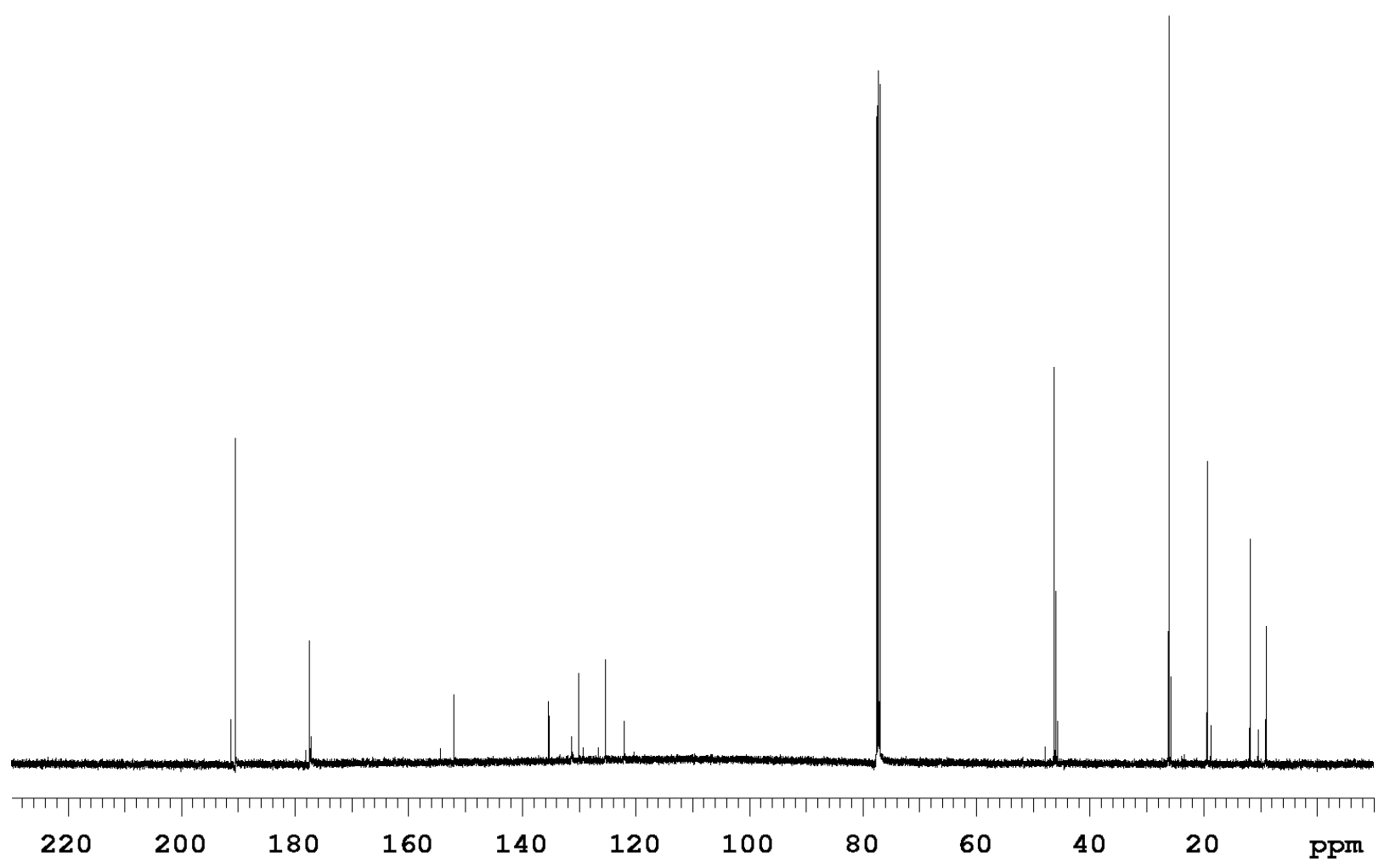


wr5p265
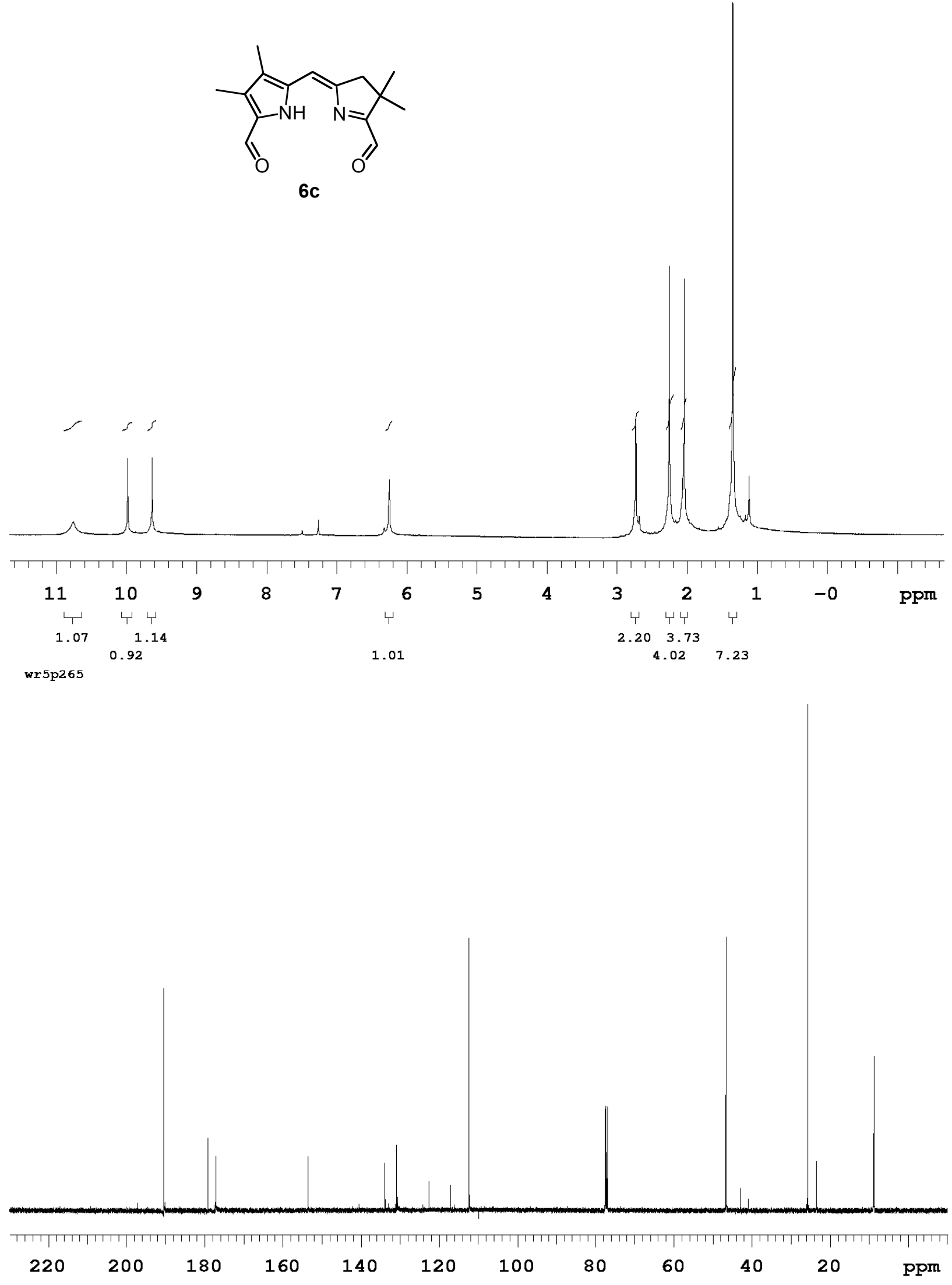
wr5p160
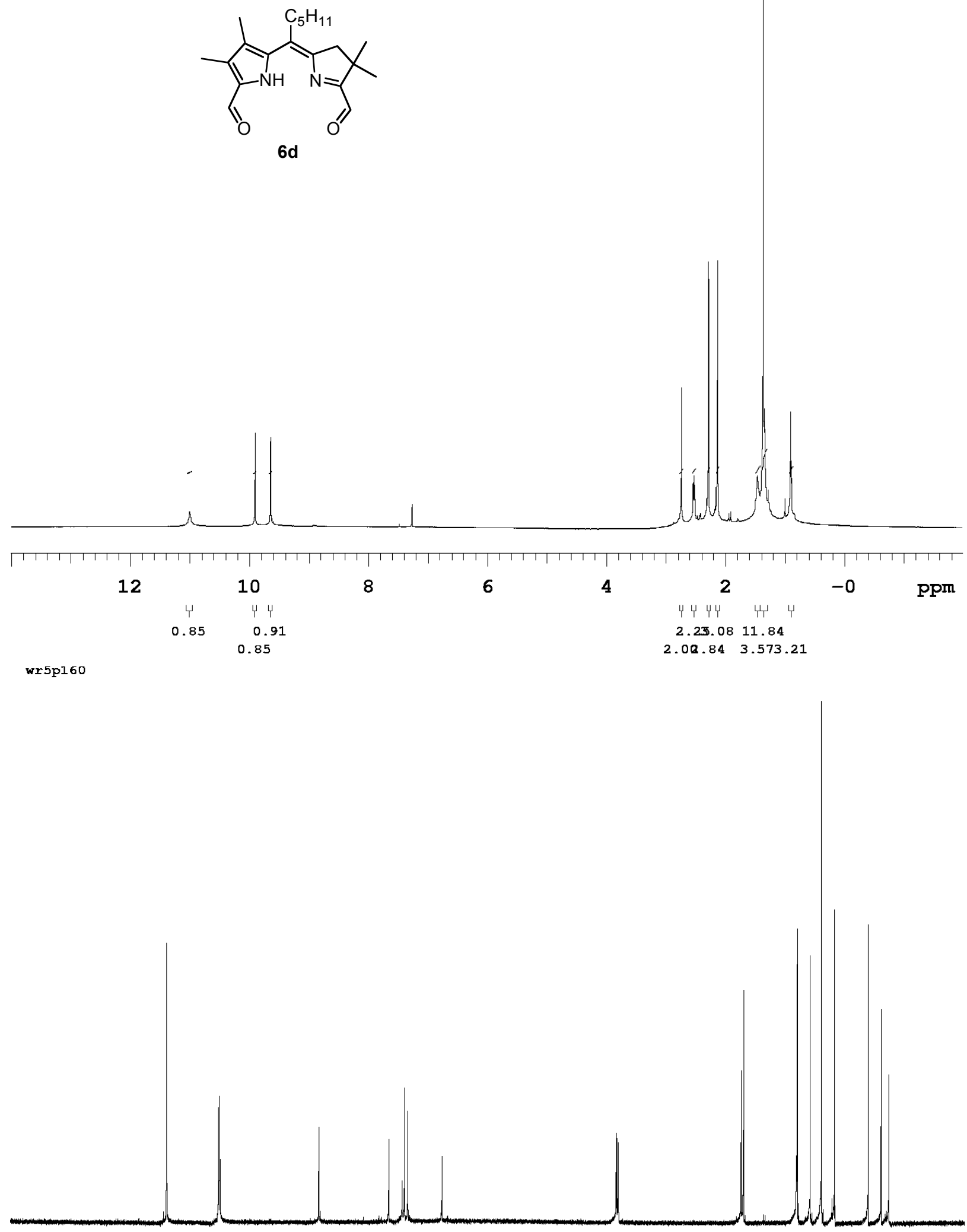

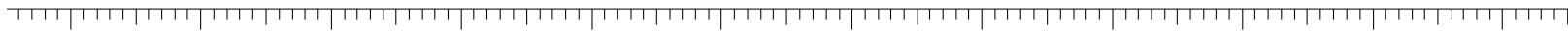
$\begin{array}{llllllllllll}220 & 200 & 180 & 160 & 140 & 120 & 100 & 80 & 60 & 40 & 20 & \text { Ppm }\end{array}$ 
wr5p216

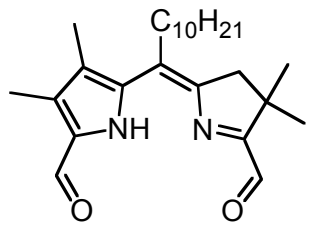

$6 e$

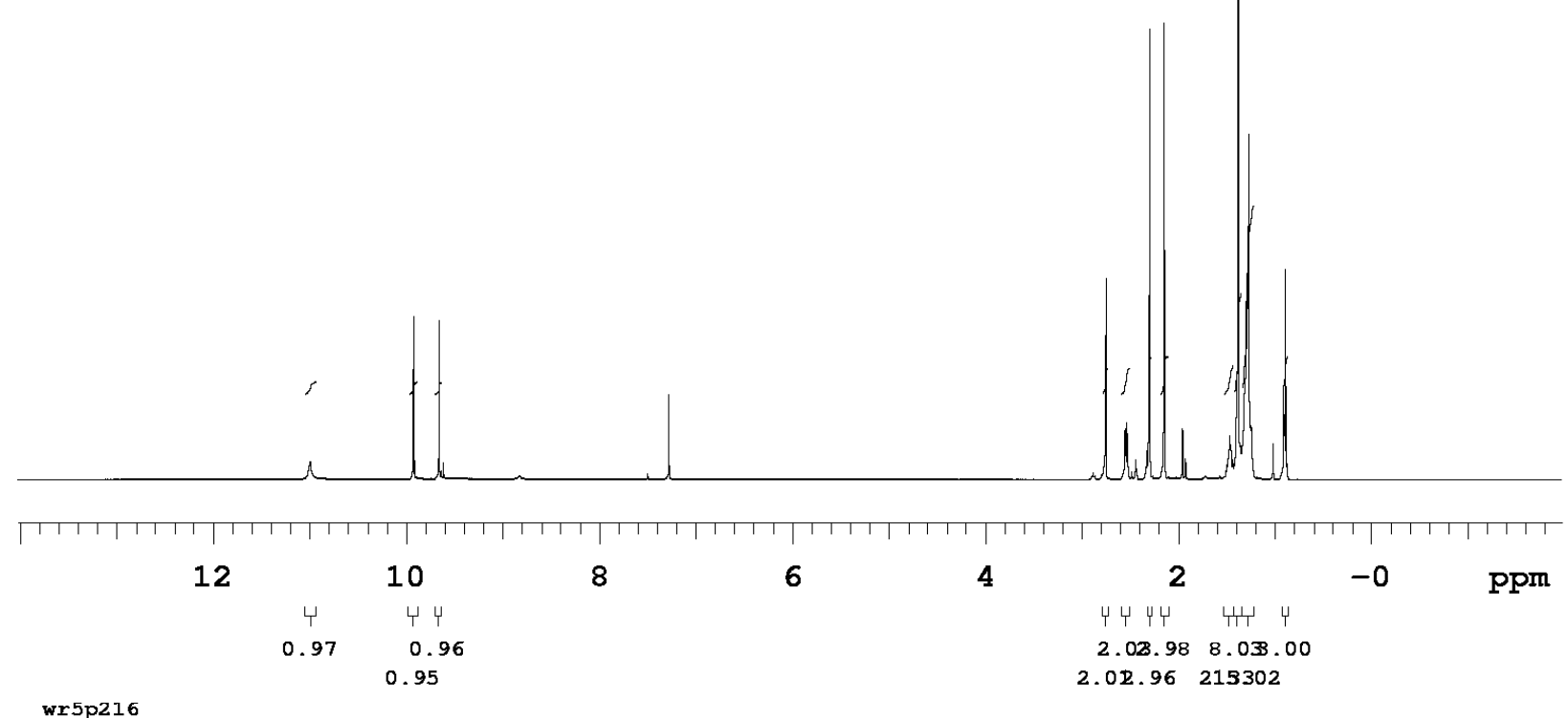

wr5p216
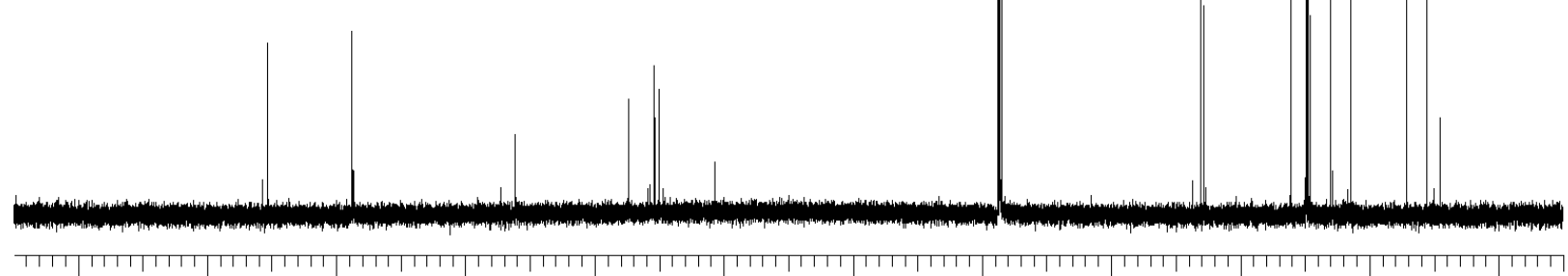
220
200
180
160
140
120
100
80
60
40
20
ppm 
wgo_1H_IV153

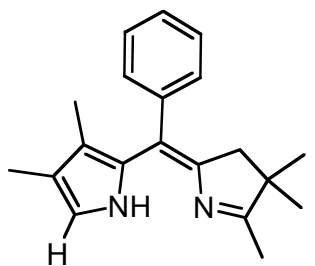

$17 a$

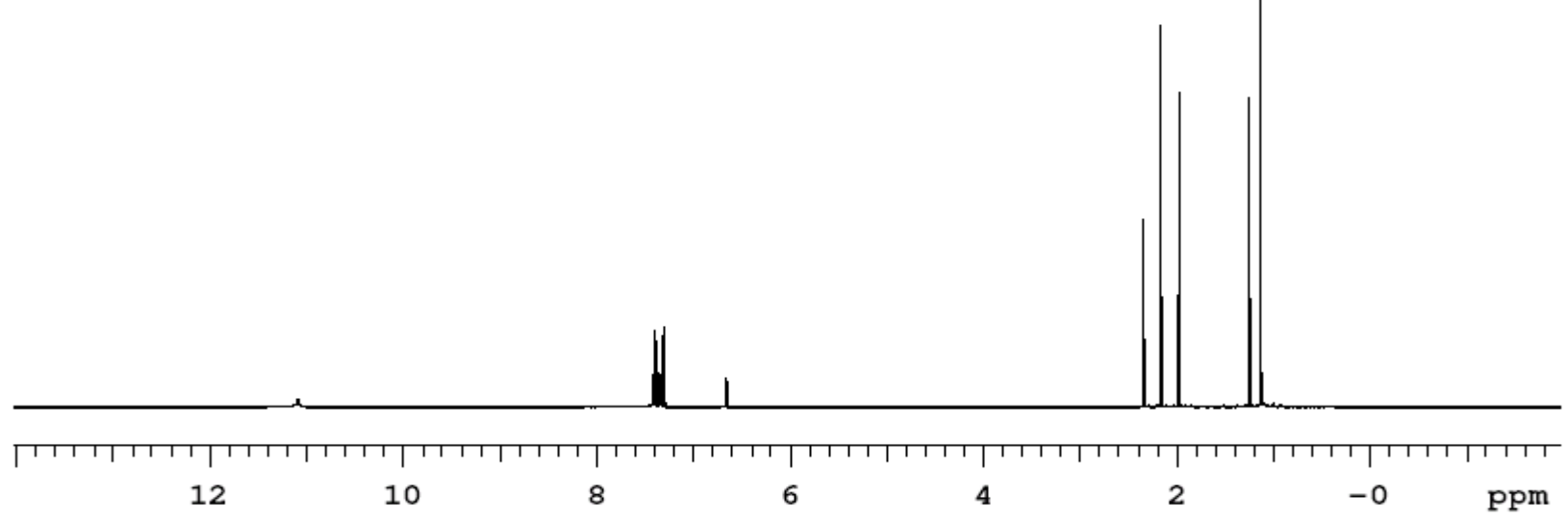

wgo_13C IV153

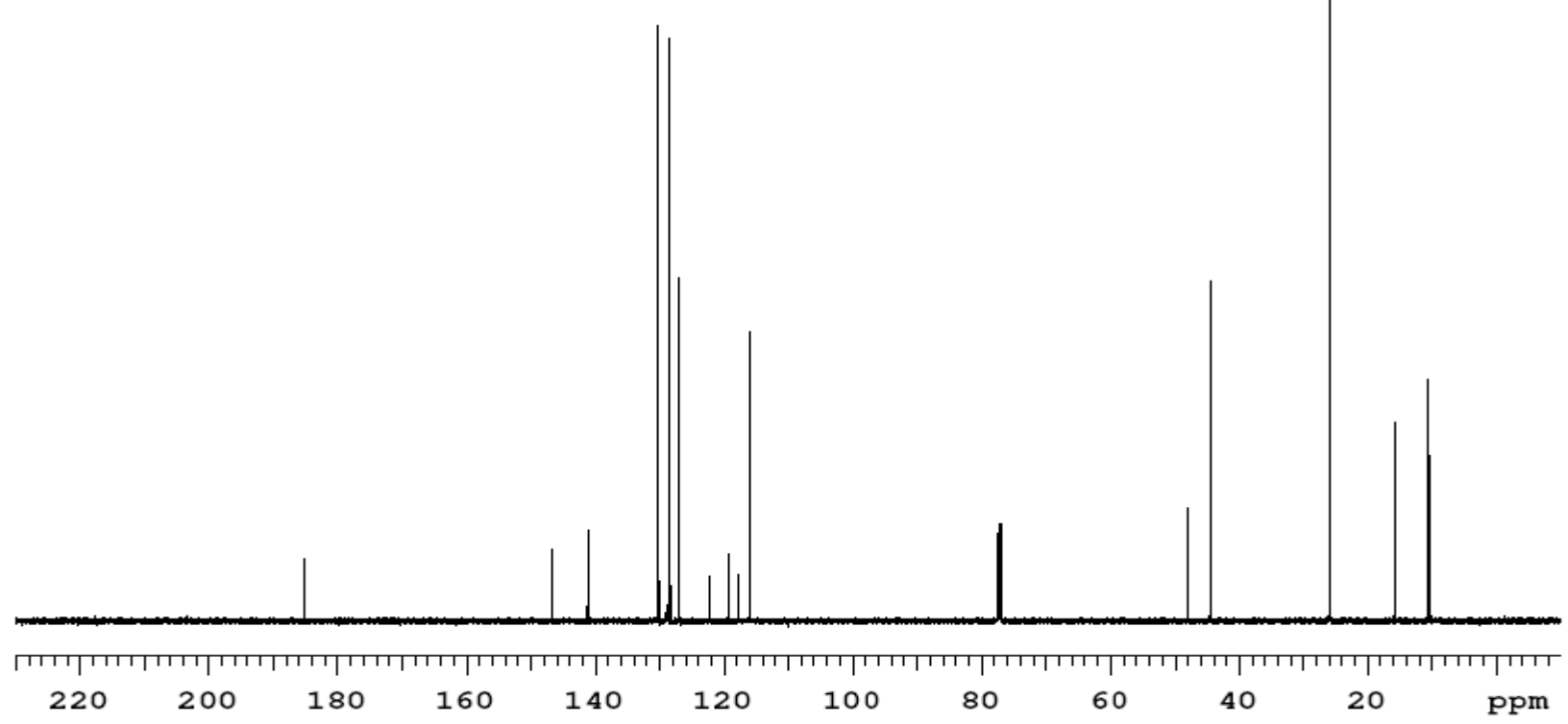


wr4p263bct14
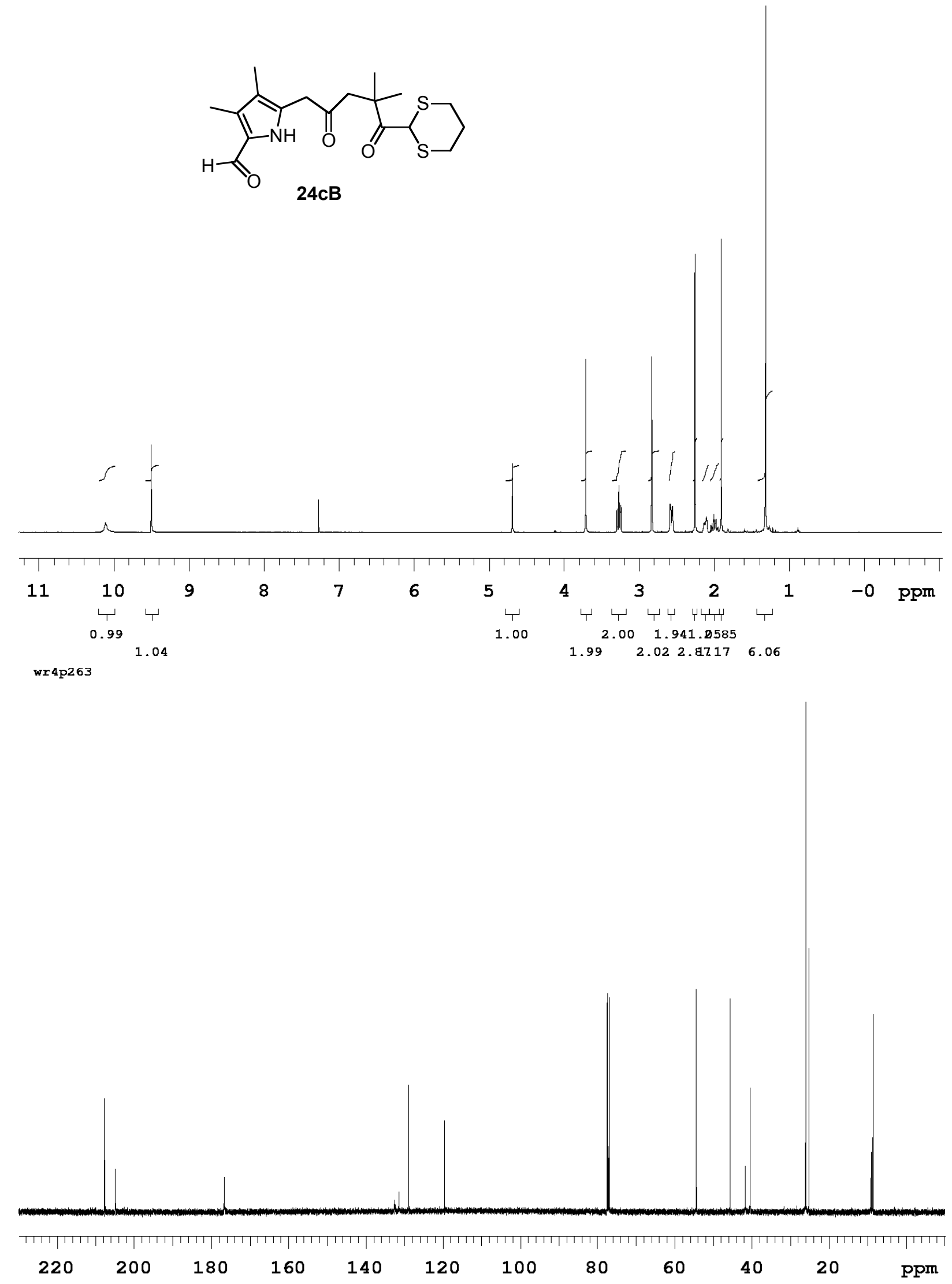
wgo_1H_III92-3

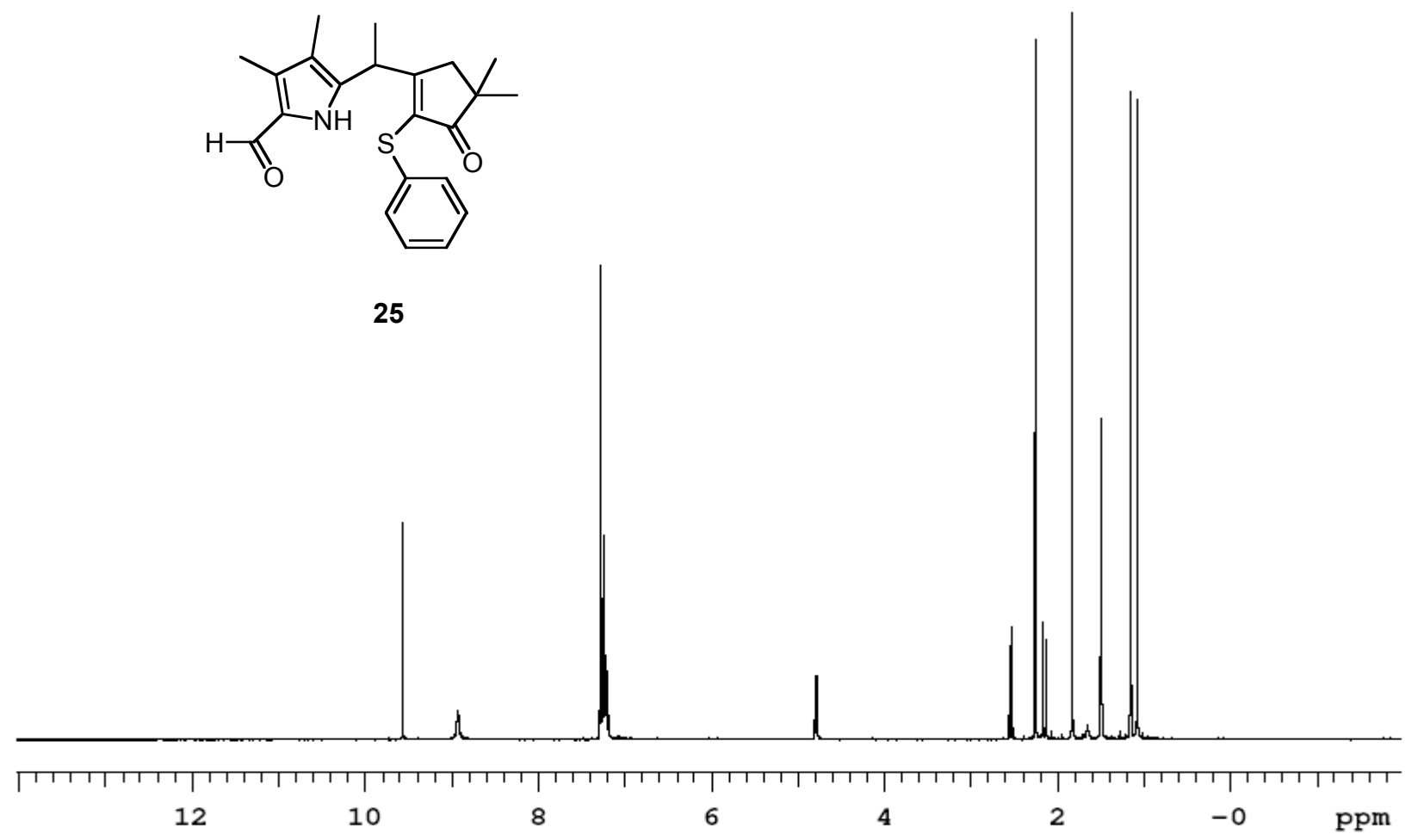

wgo 13 C III 92

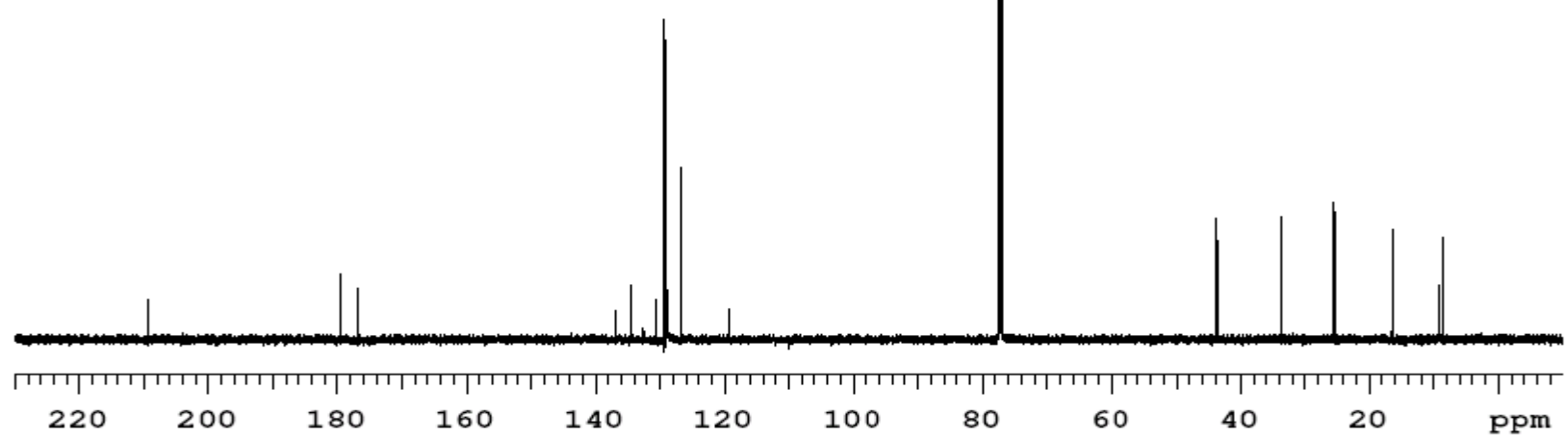



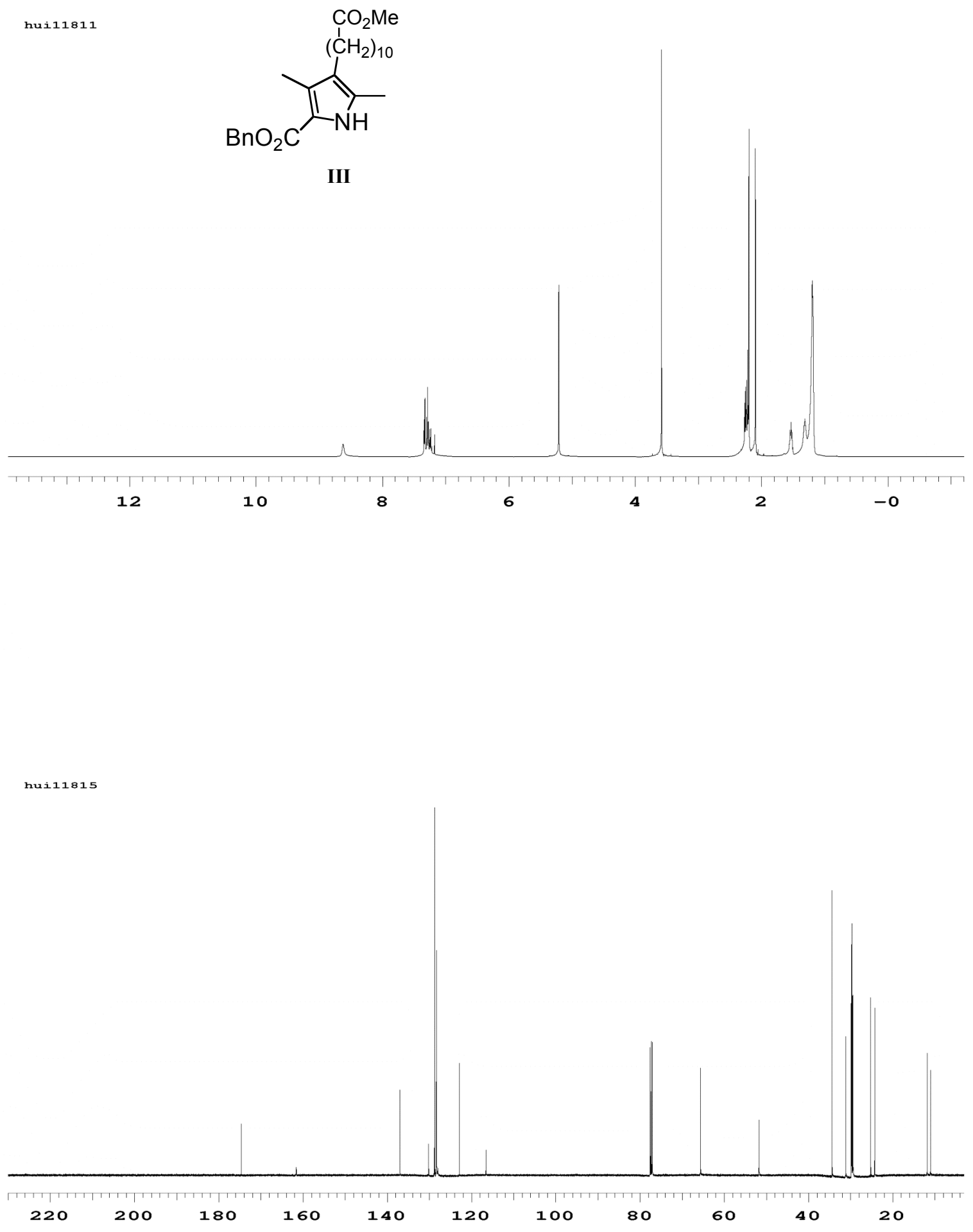

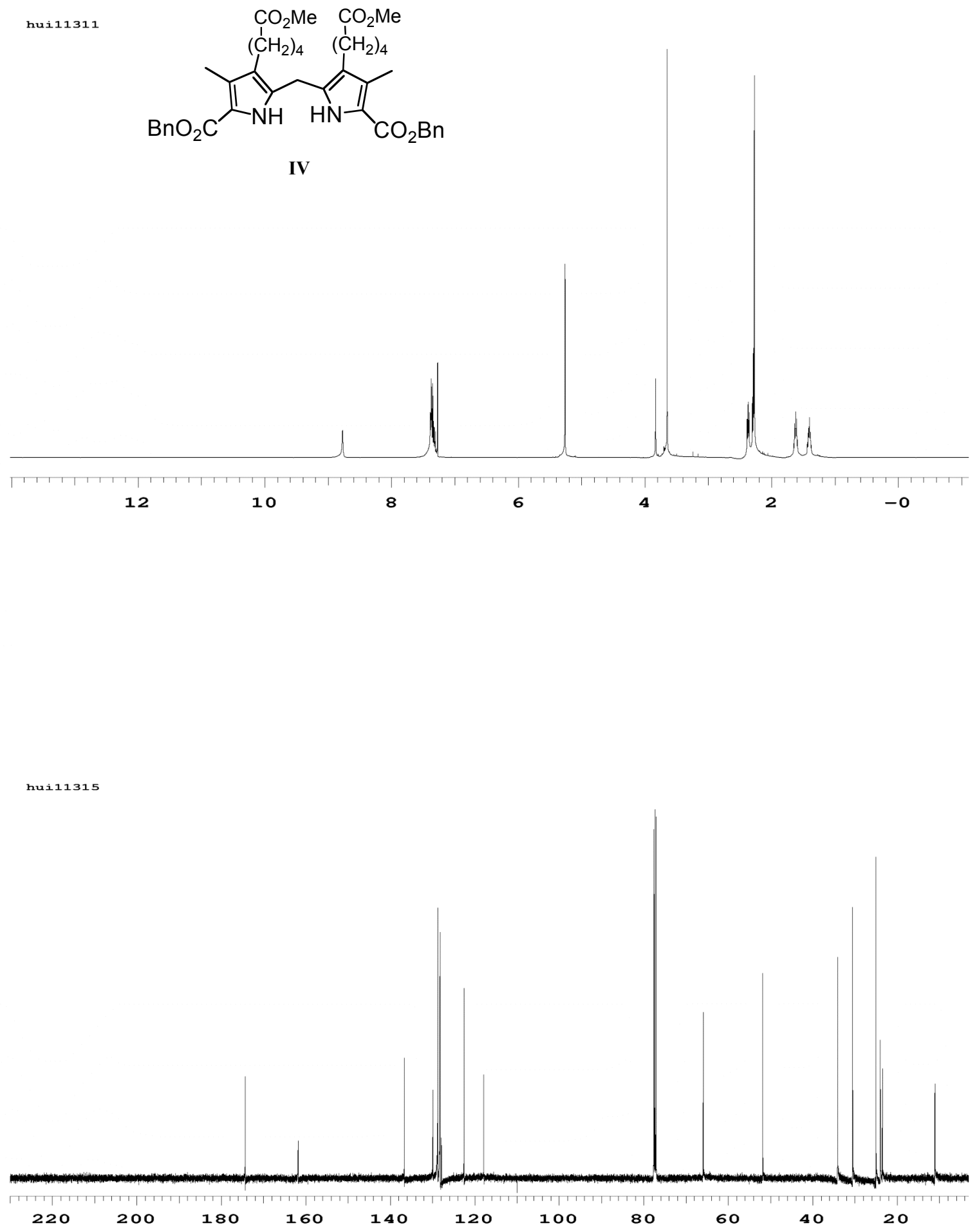

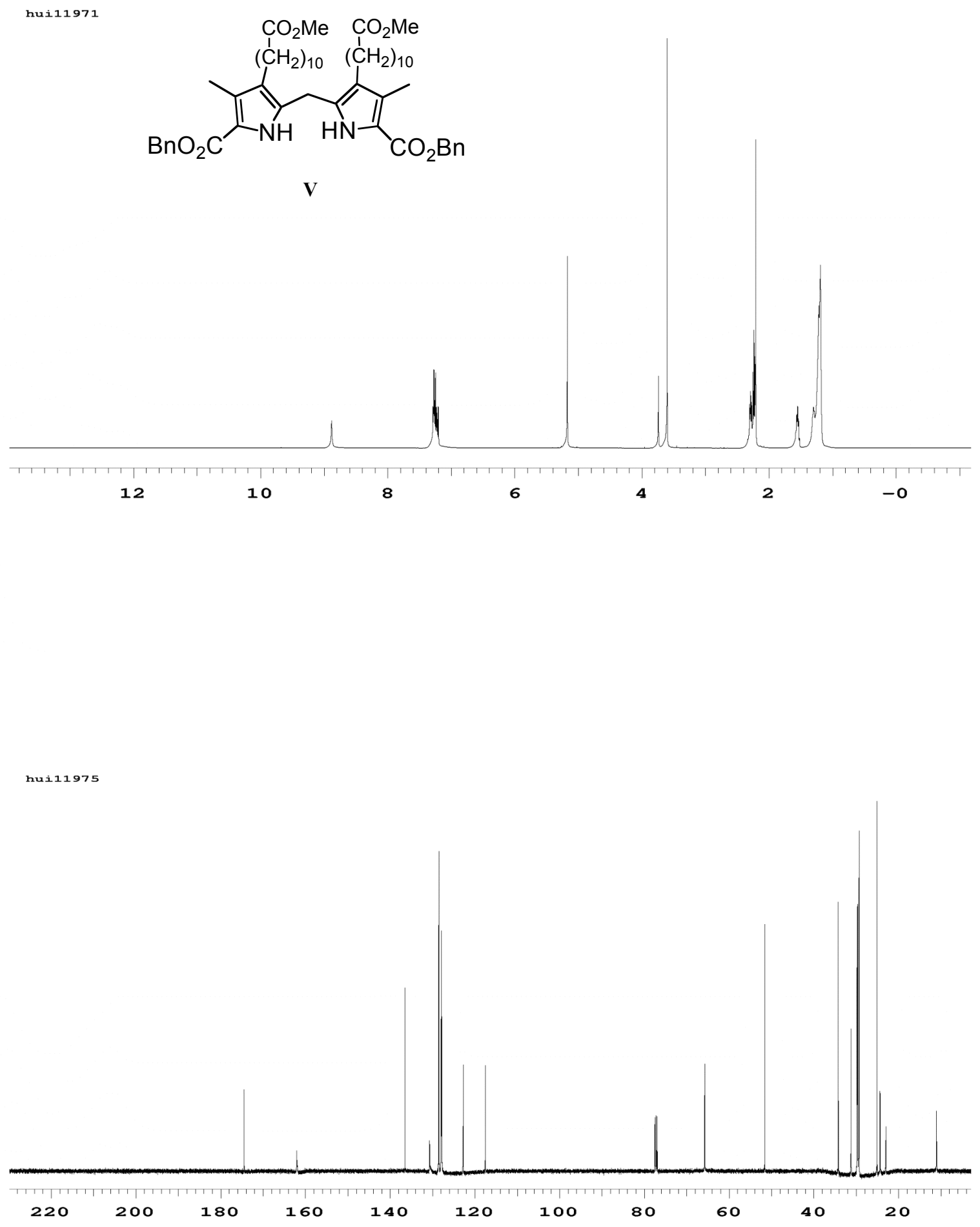


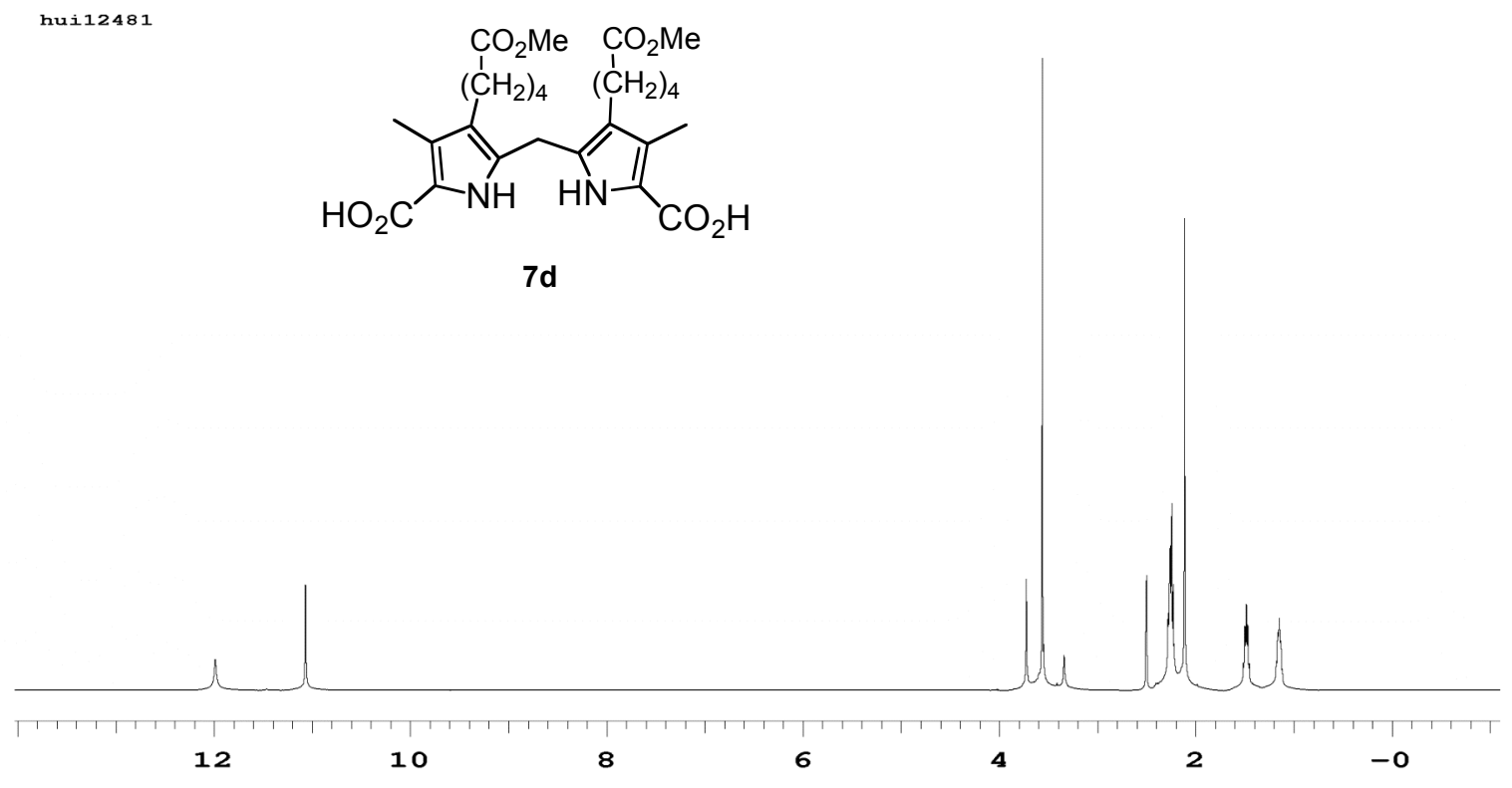

hui12485

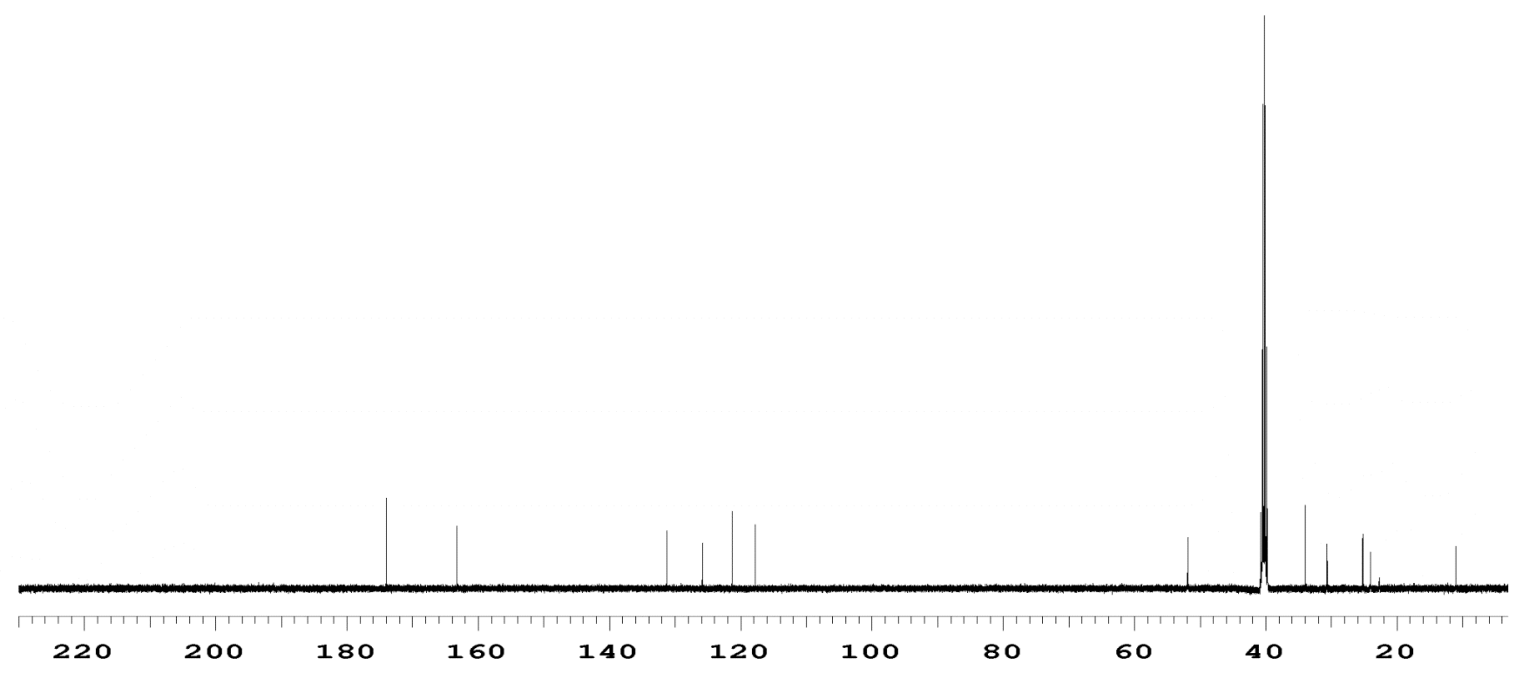




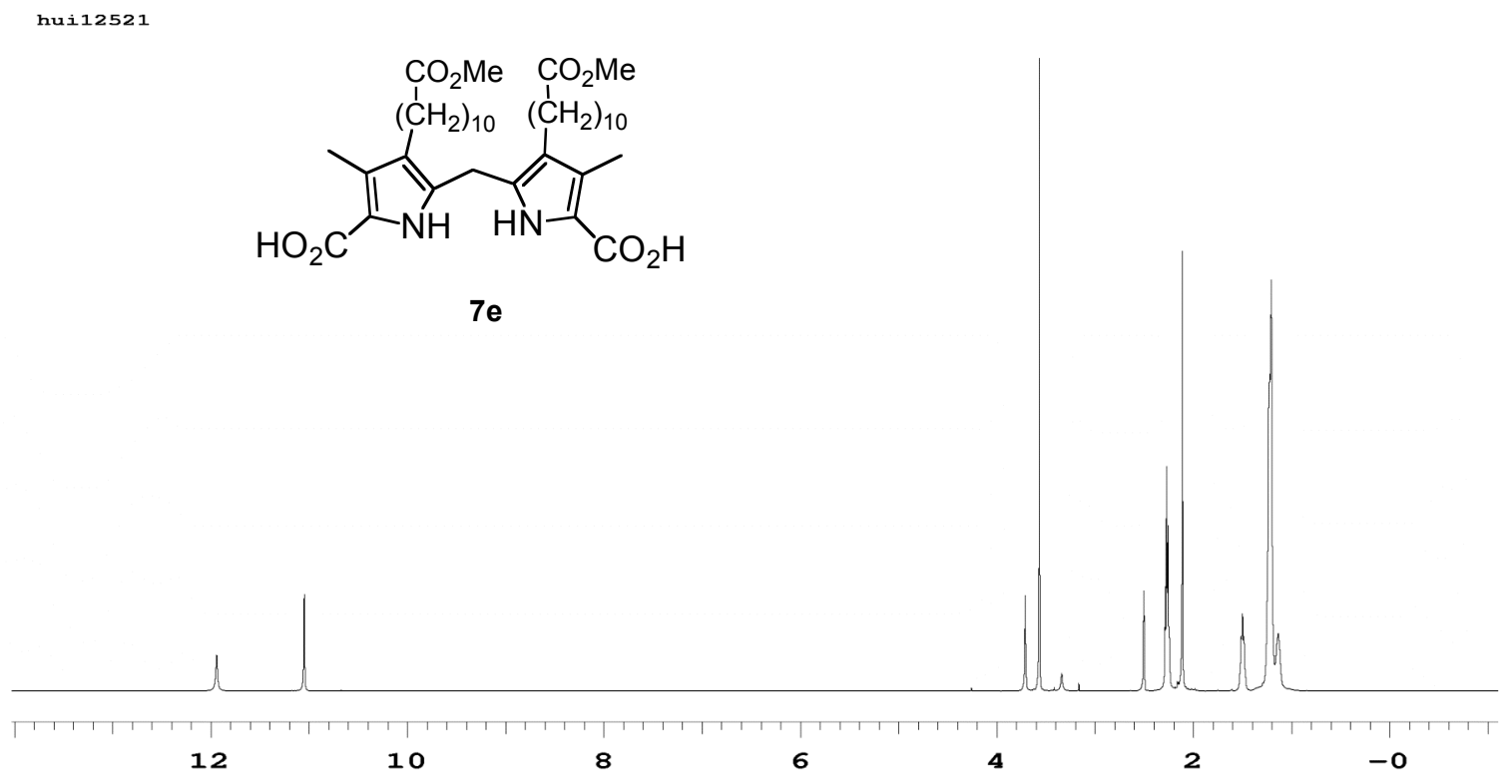

hui12525

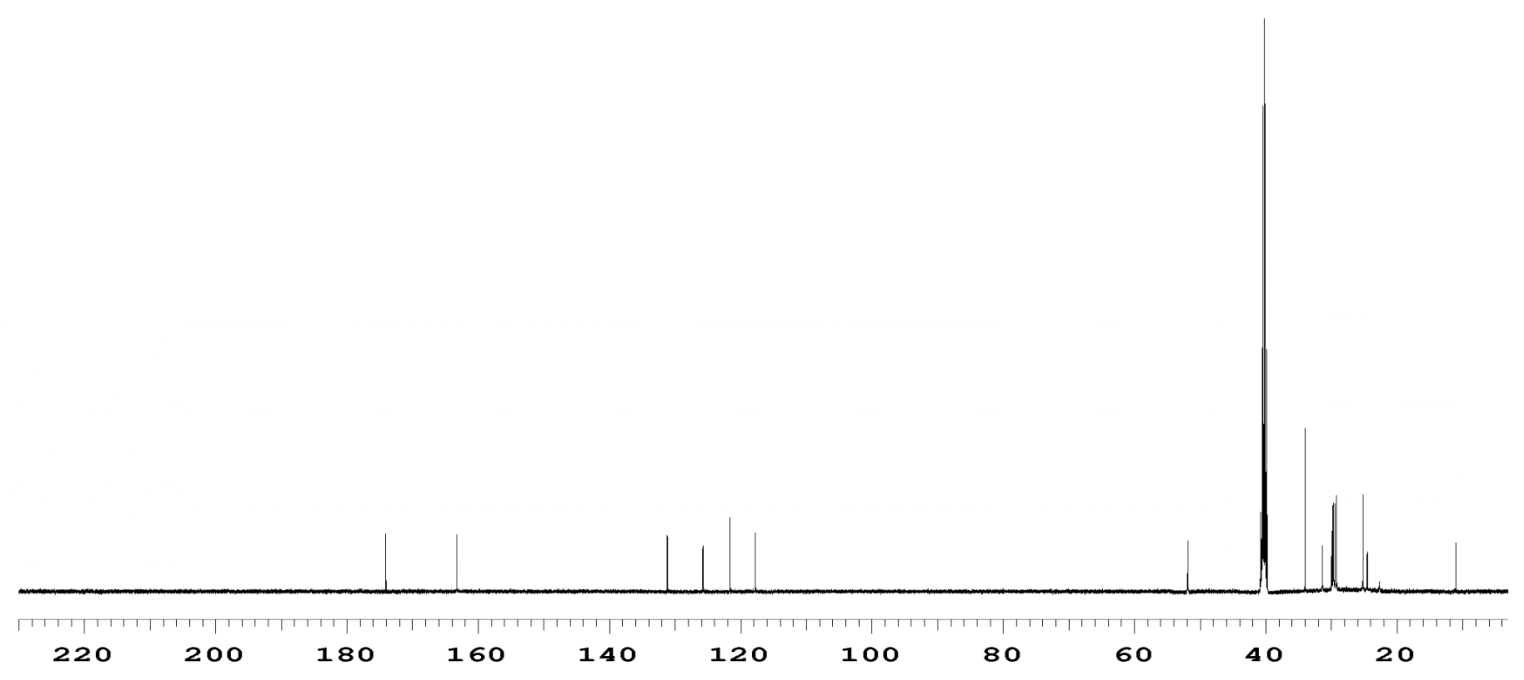


wr6p261c×4
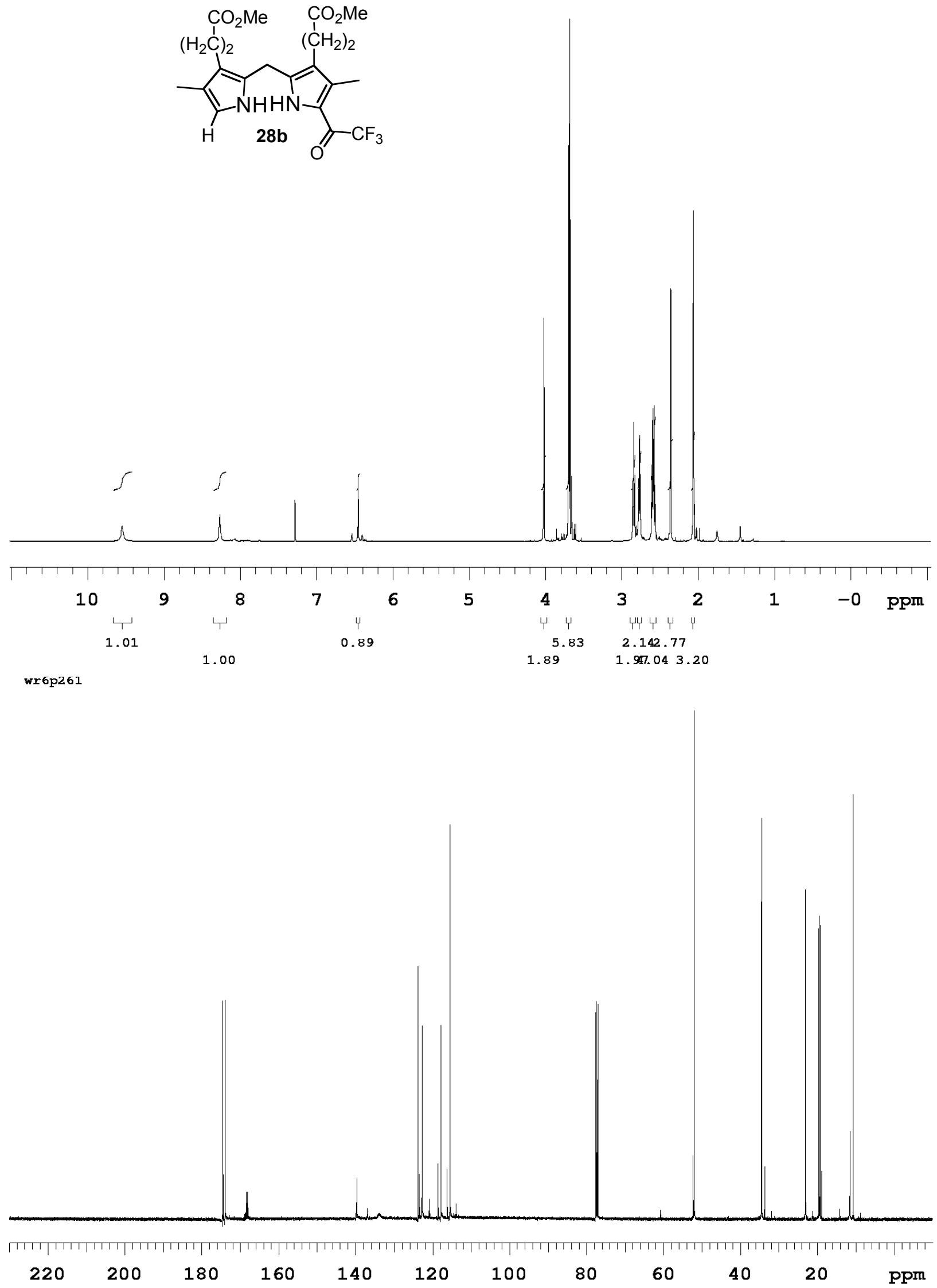

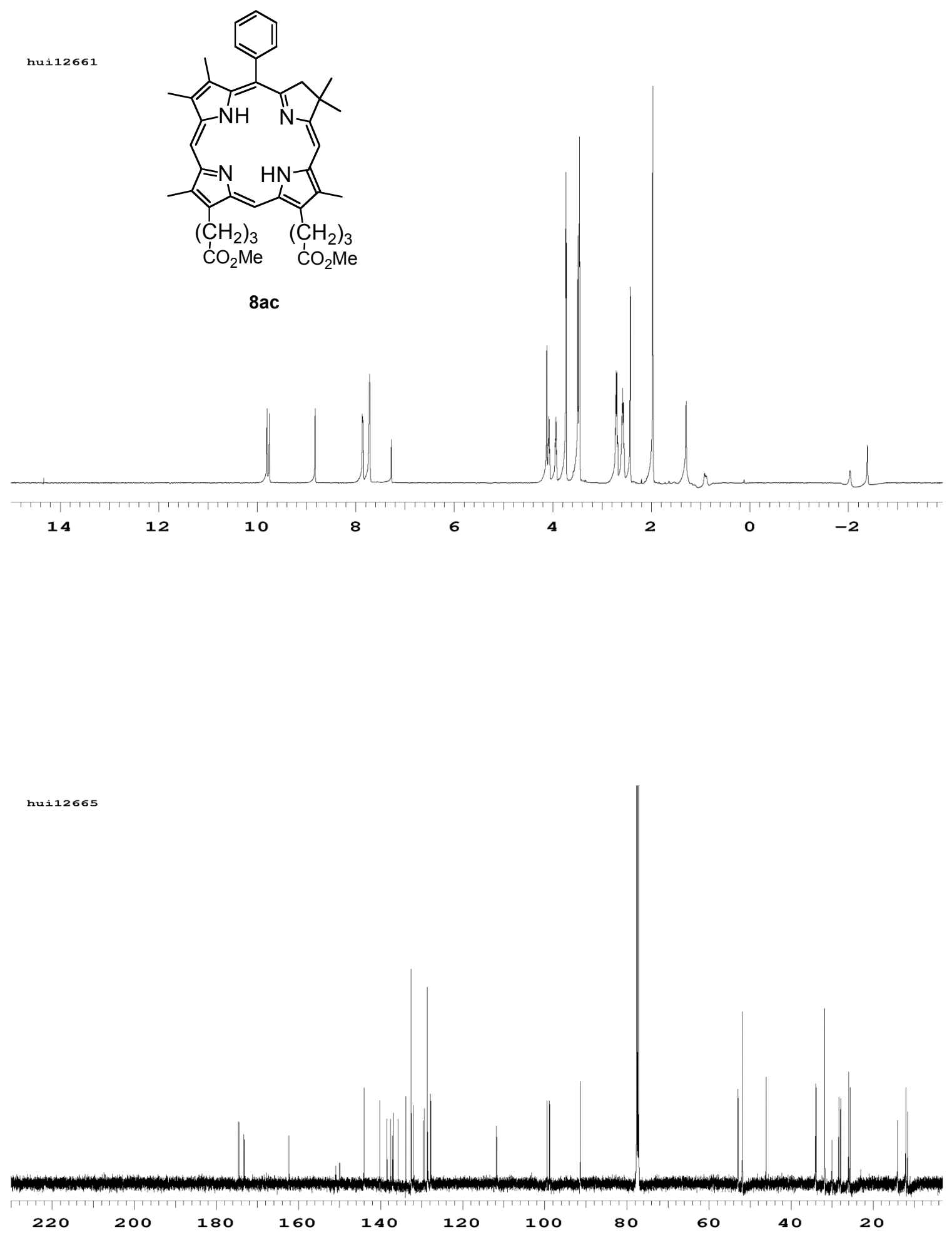

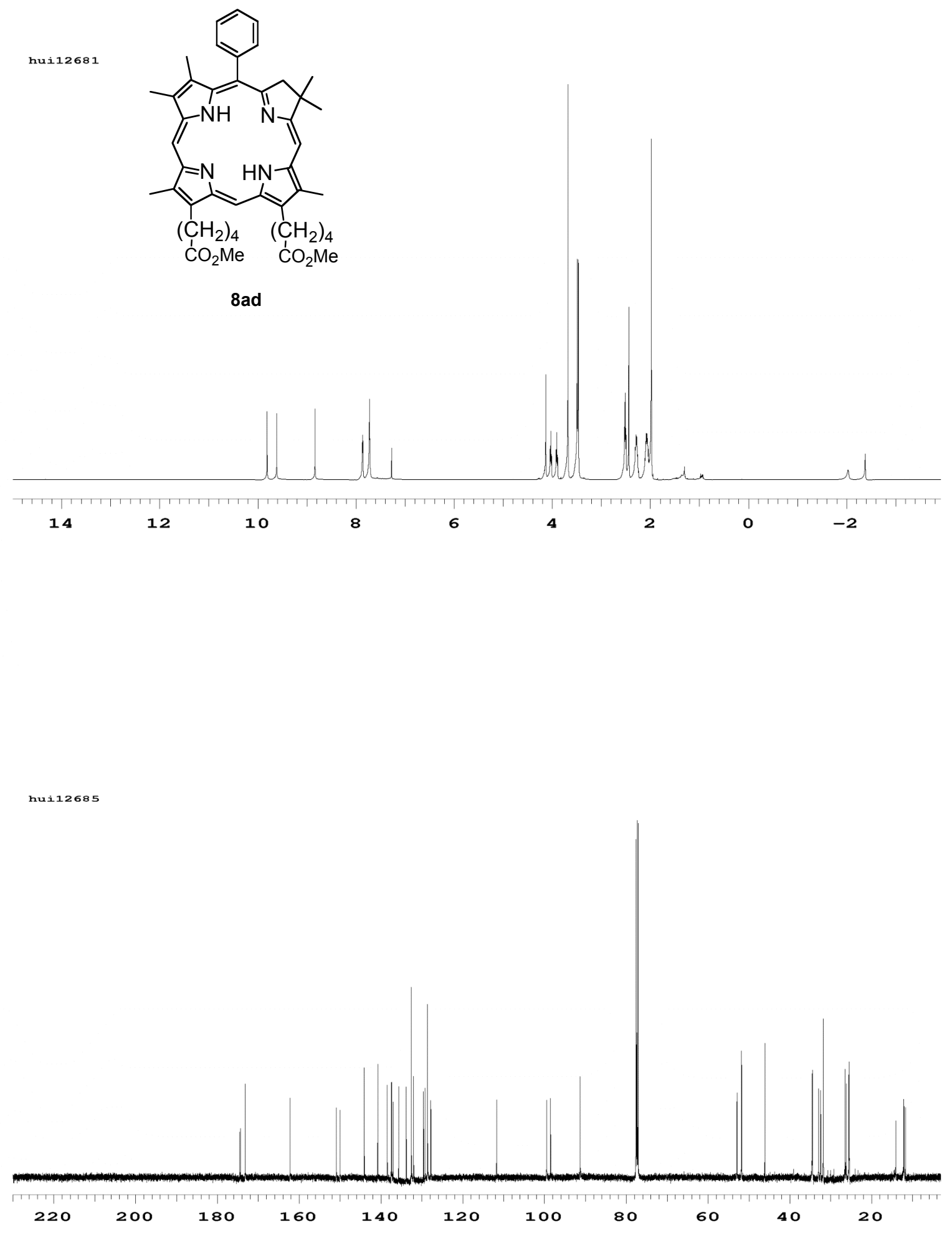

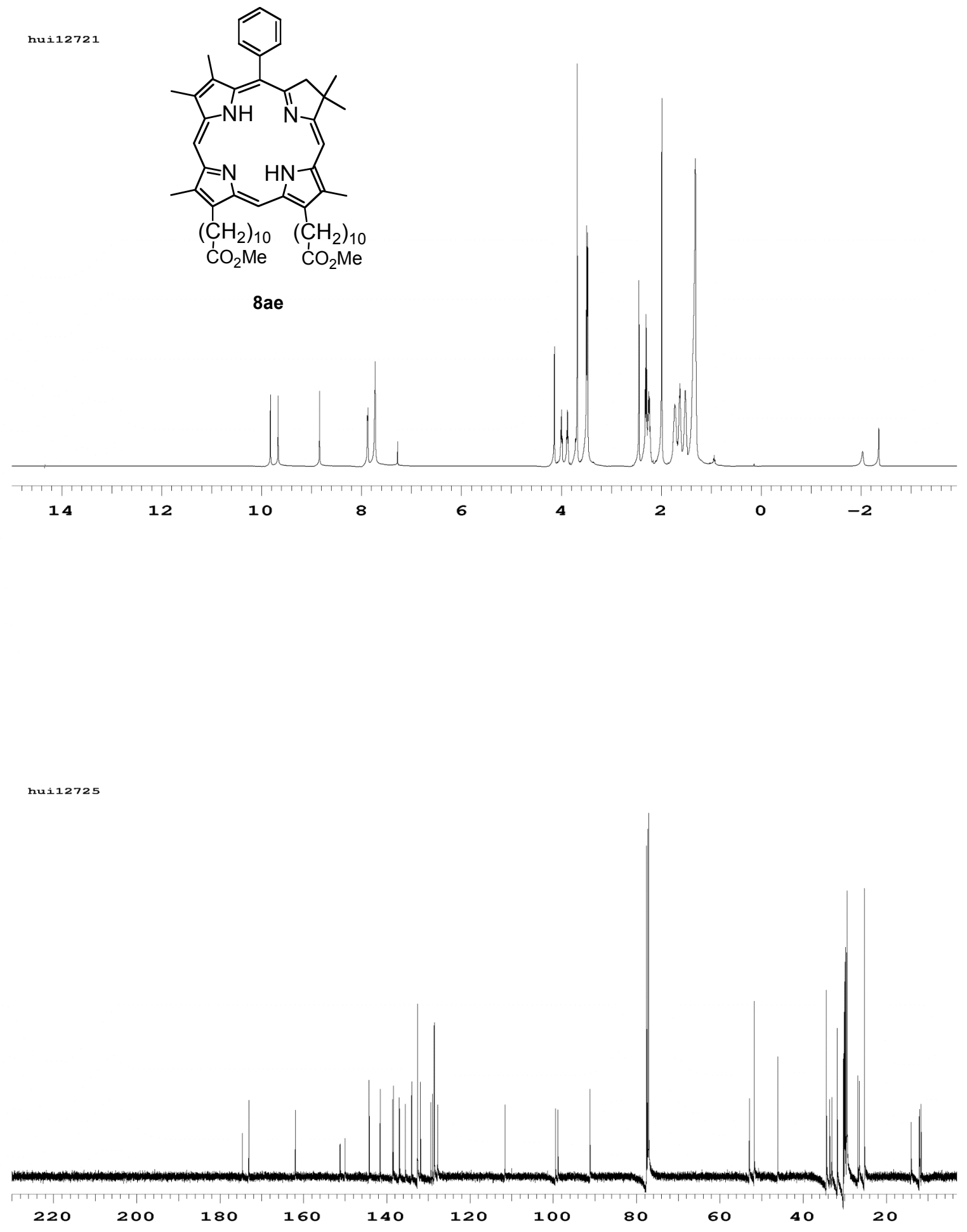

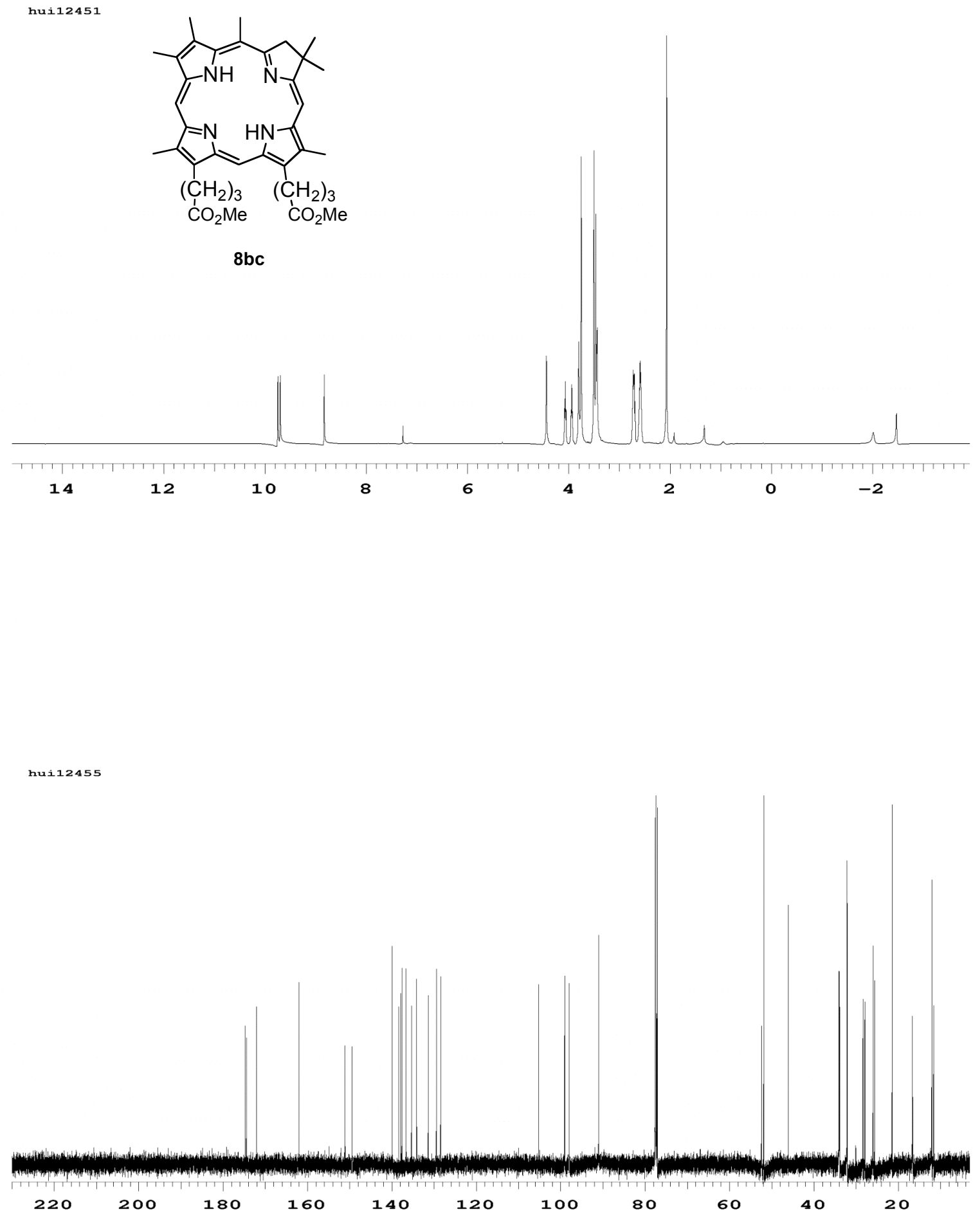

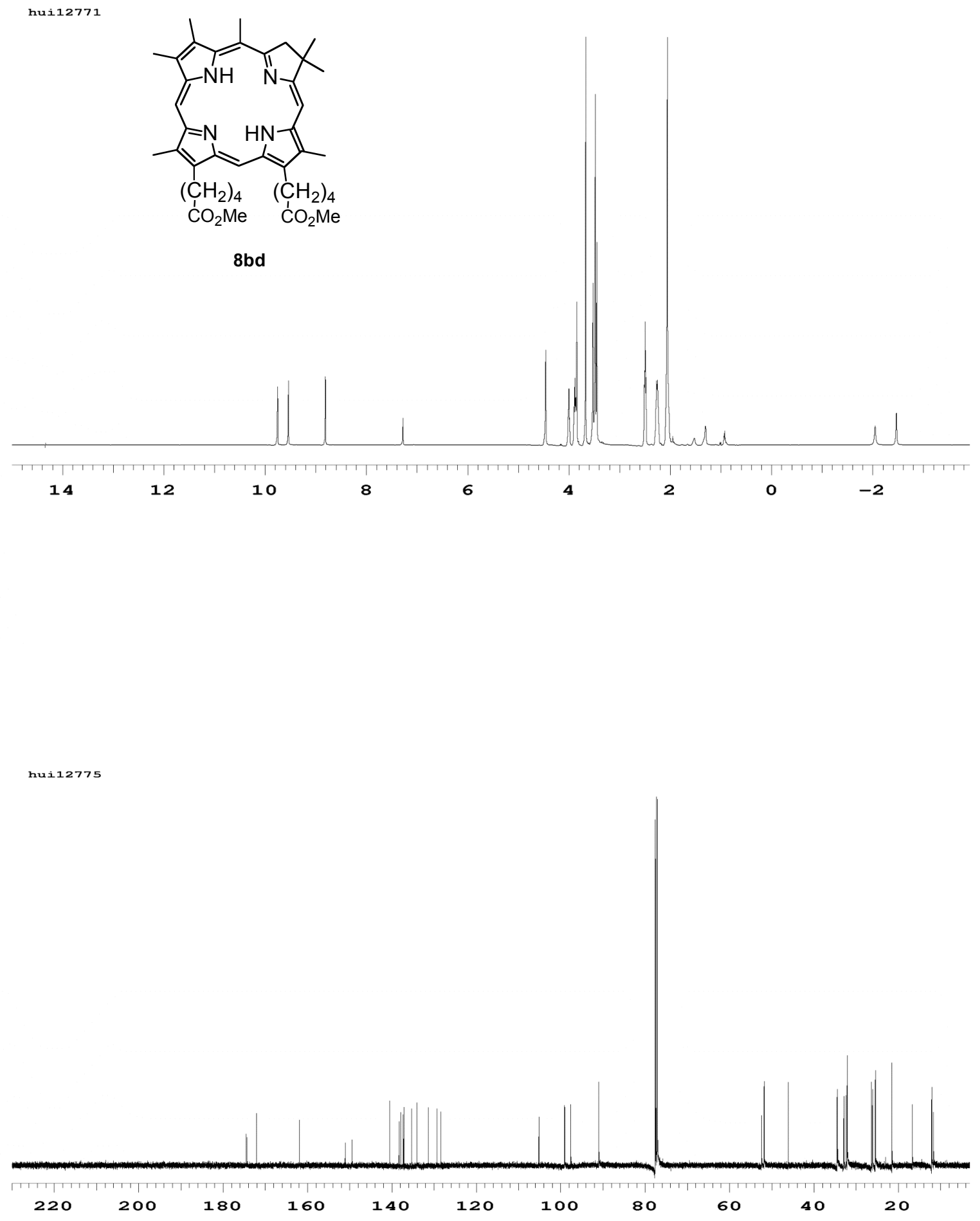

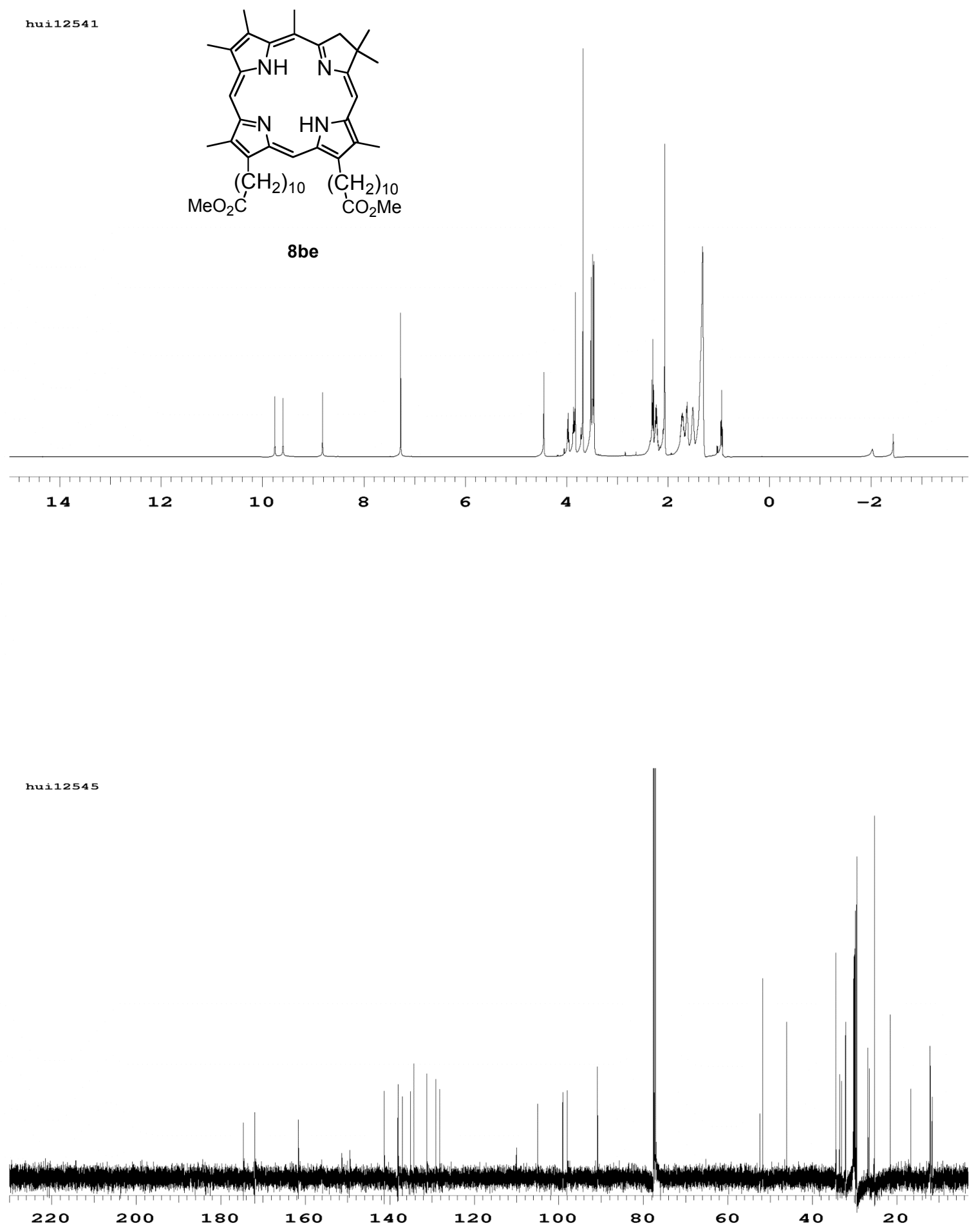


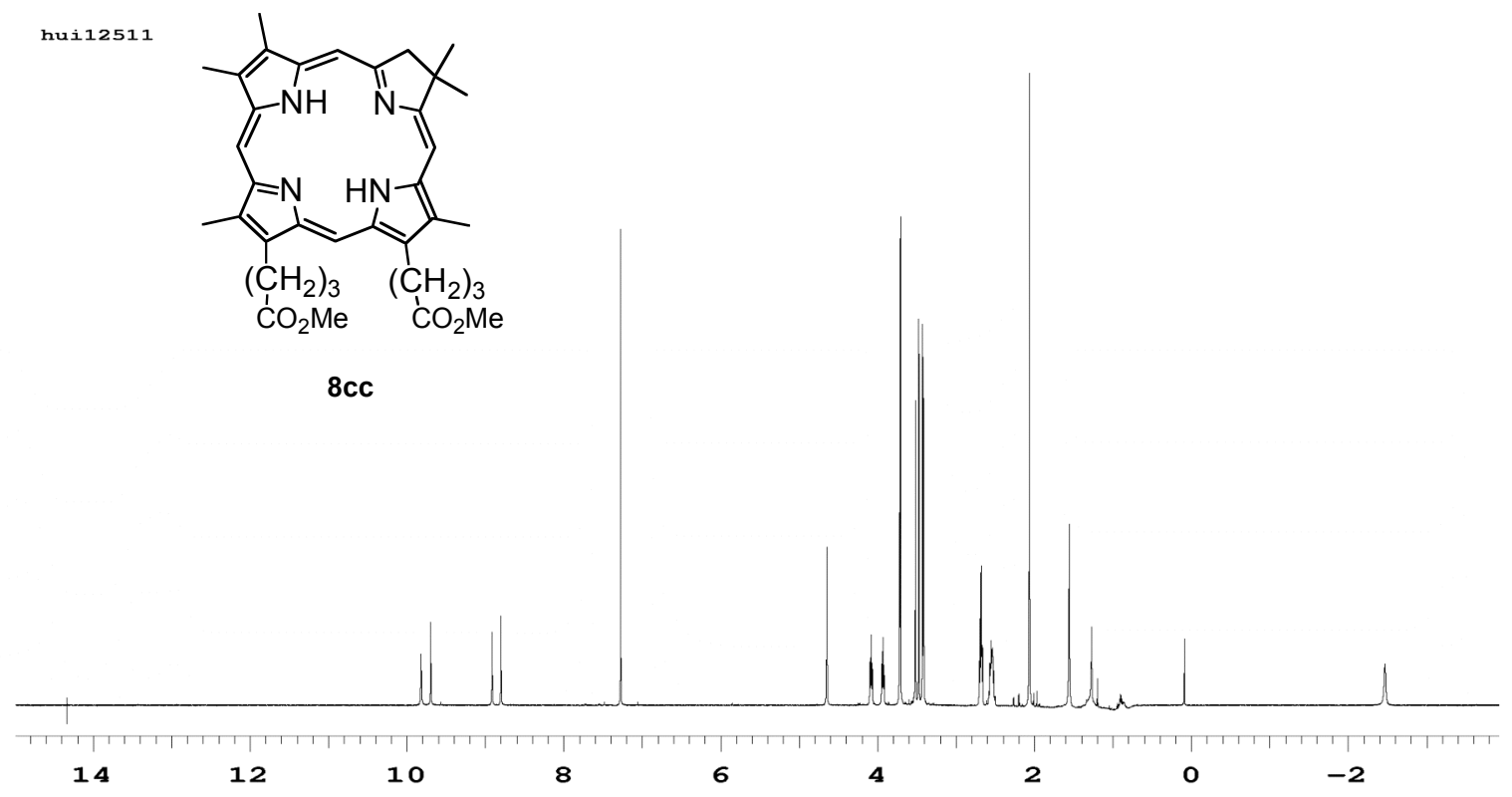

hui12915

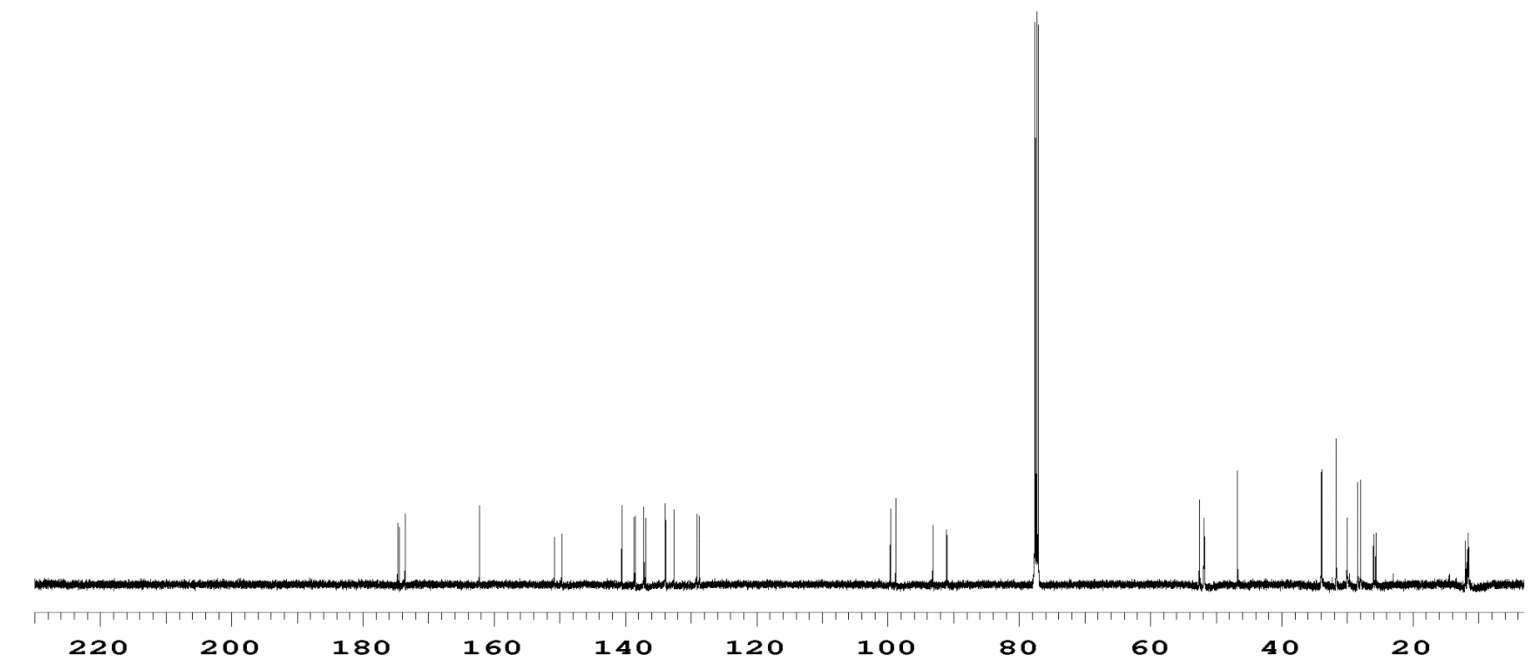



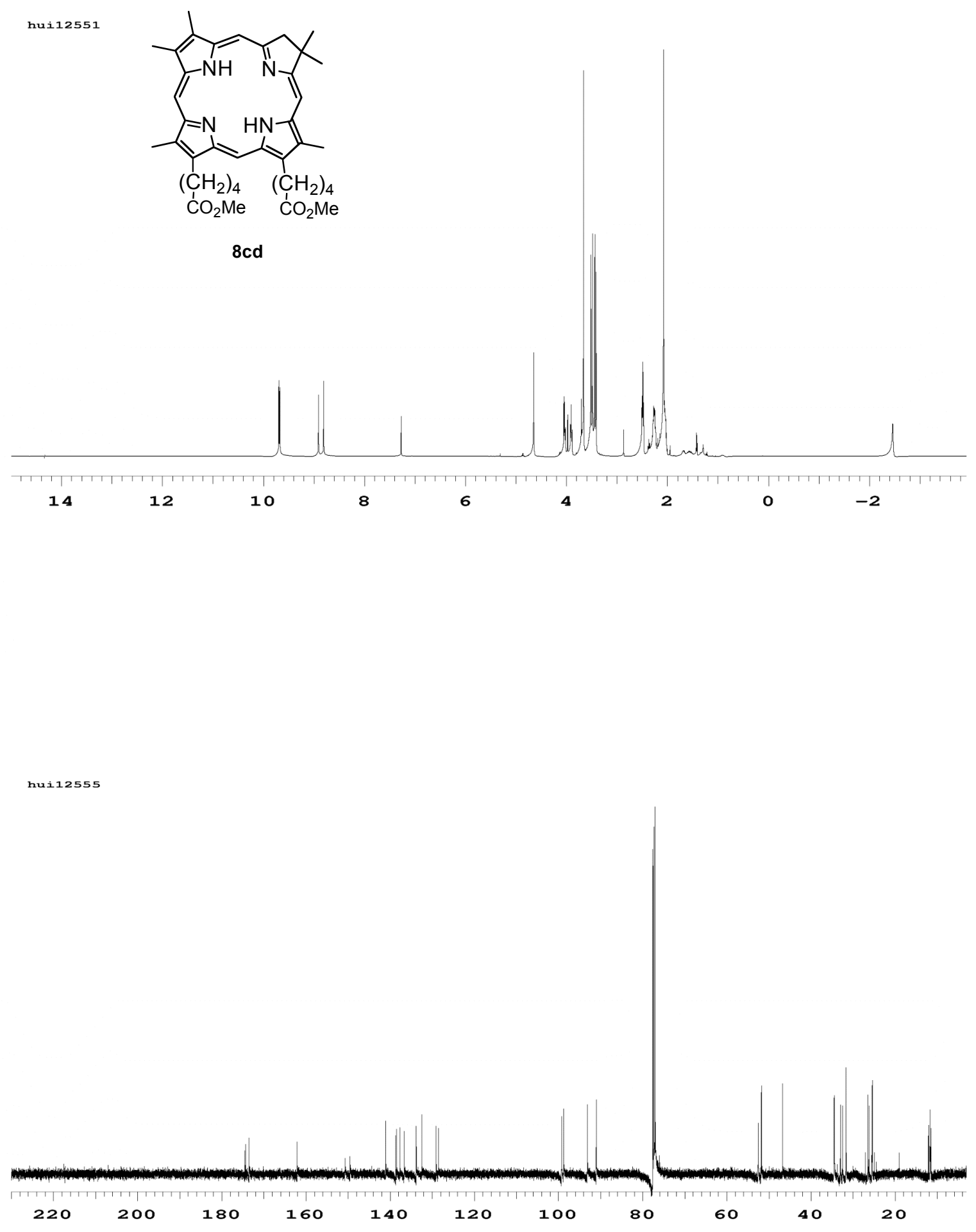


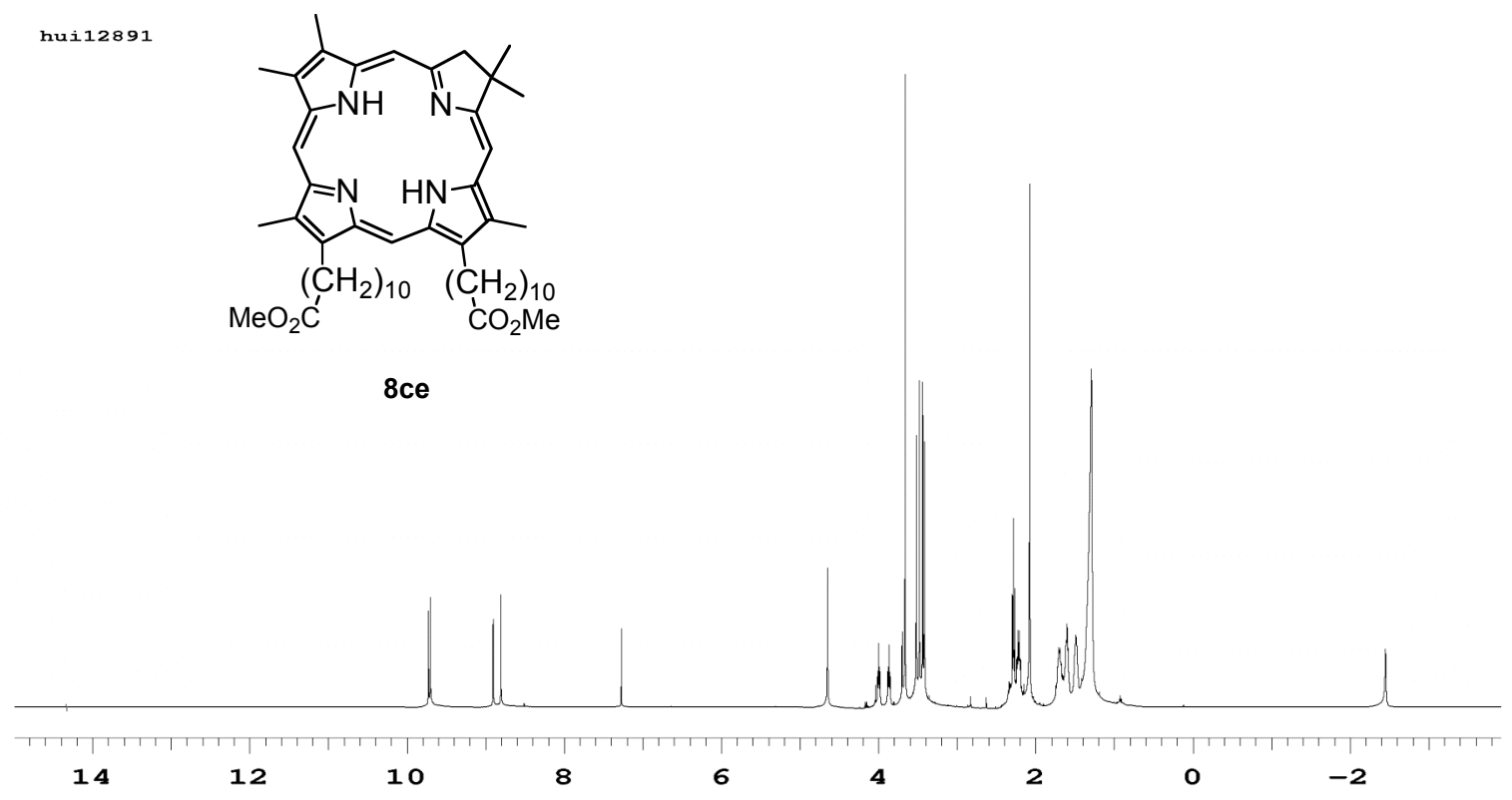

hui12895

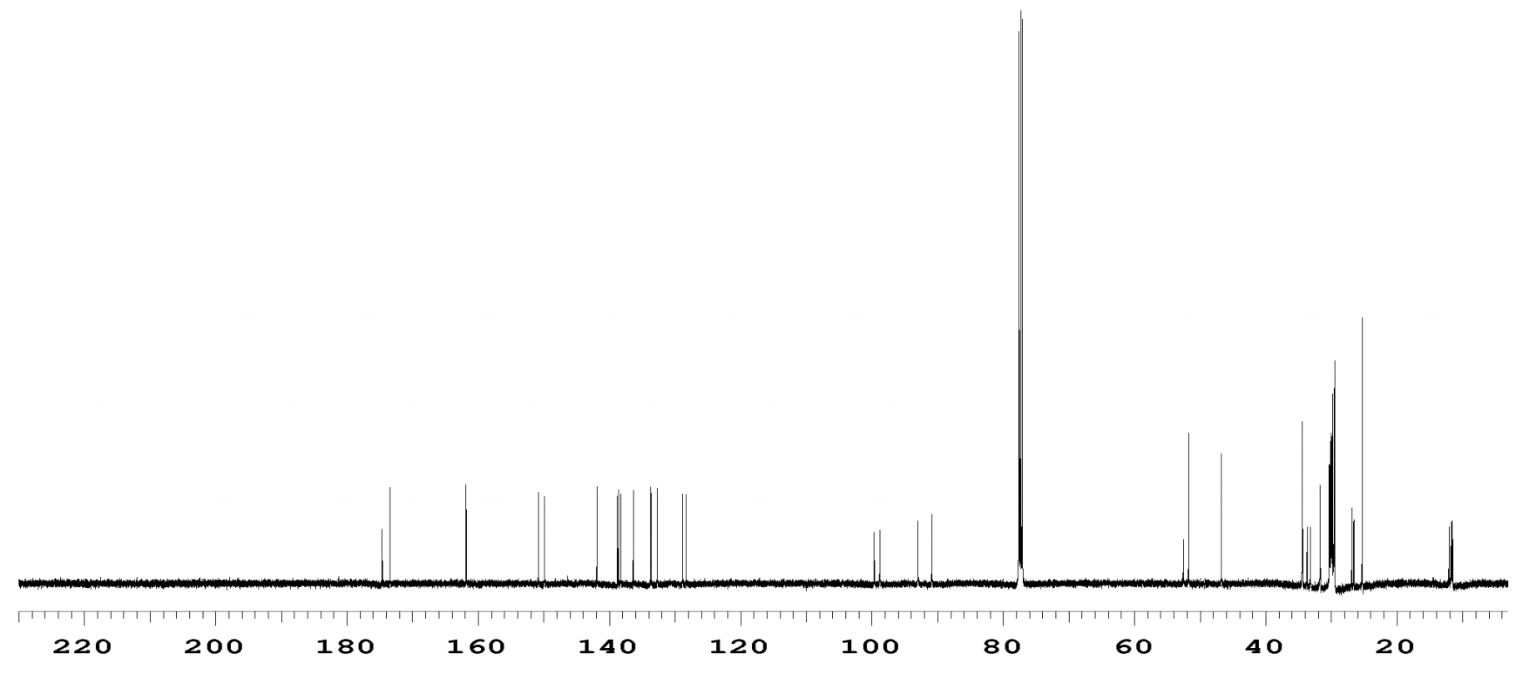


wr5p168

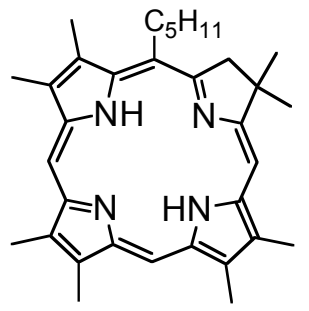

8da
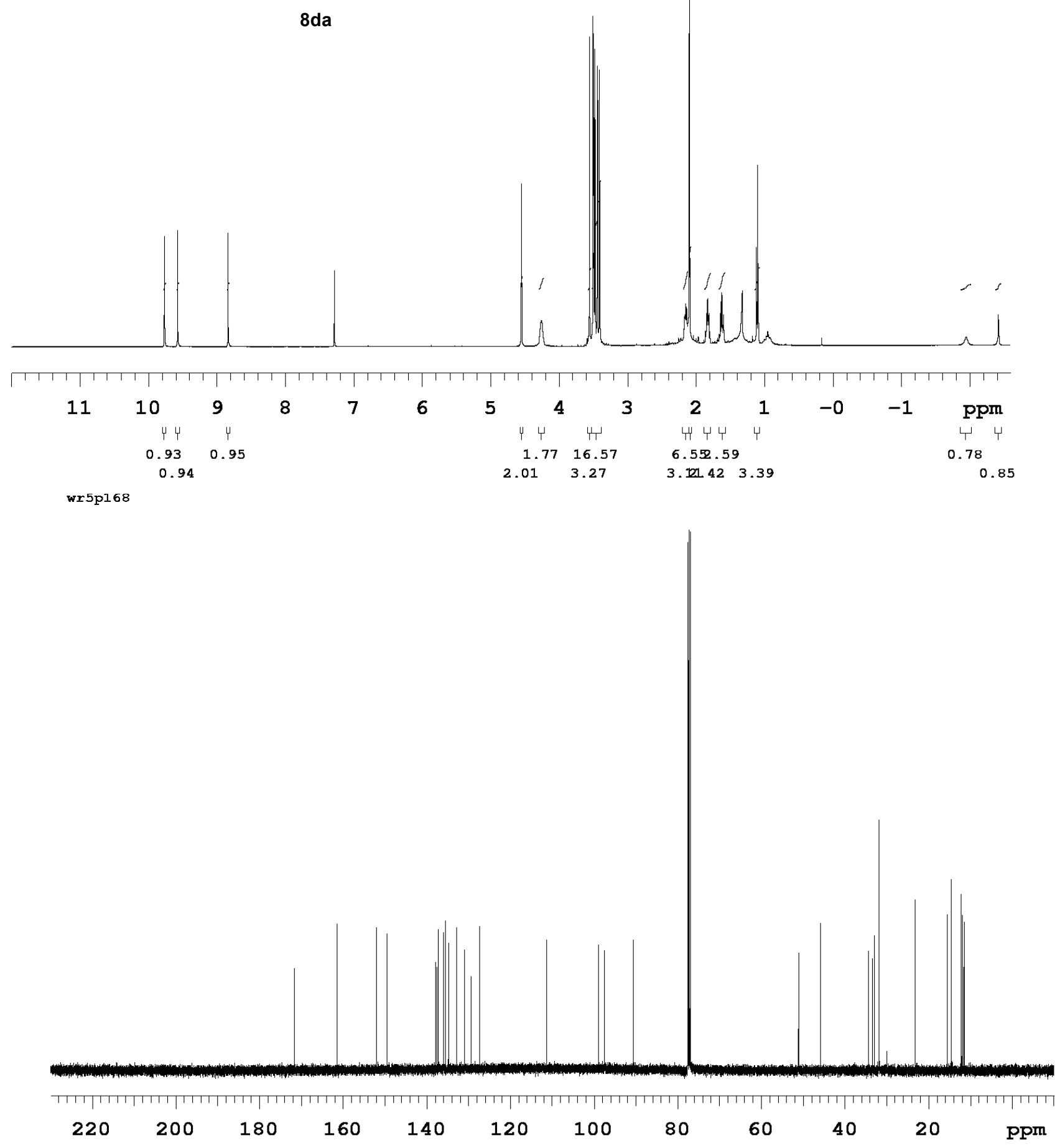
wr5p165
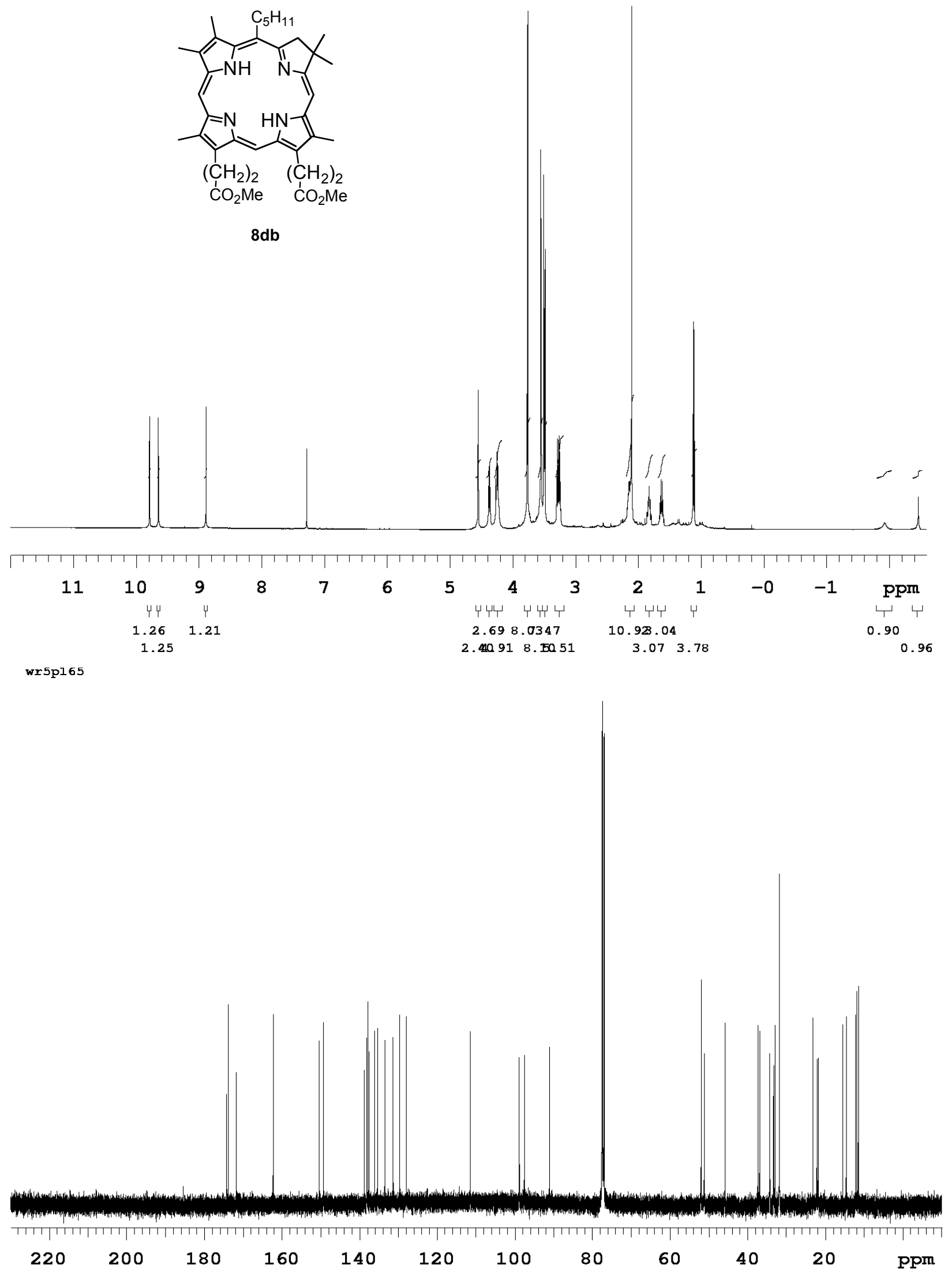
wgo_1H_IV144
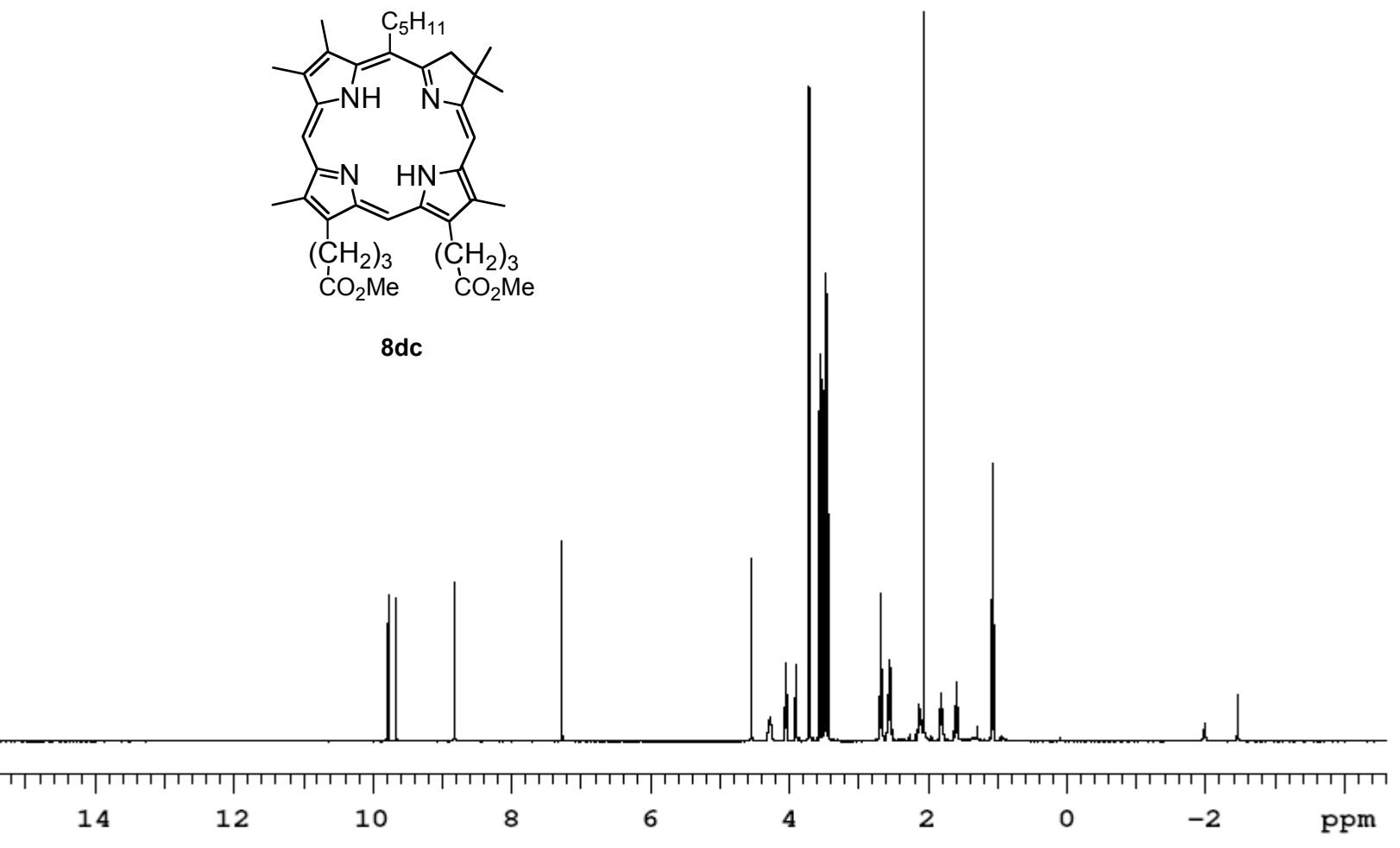

wgo_13C_IV144

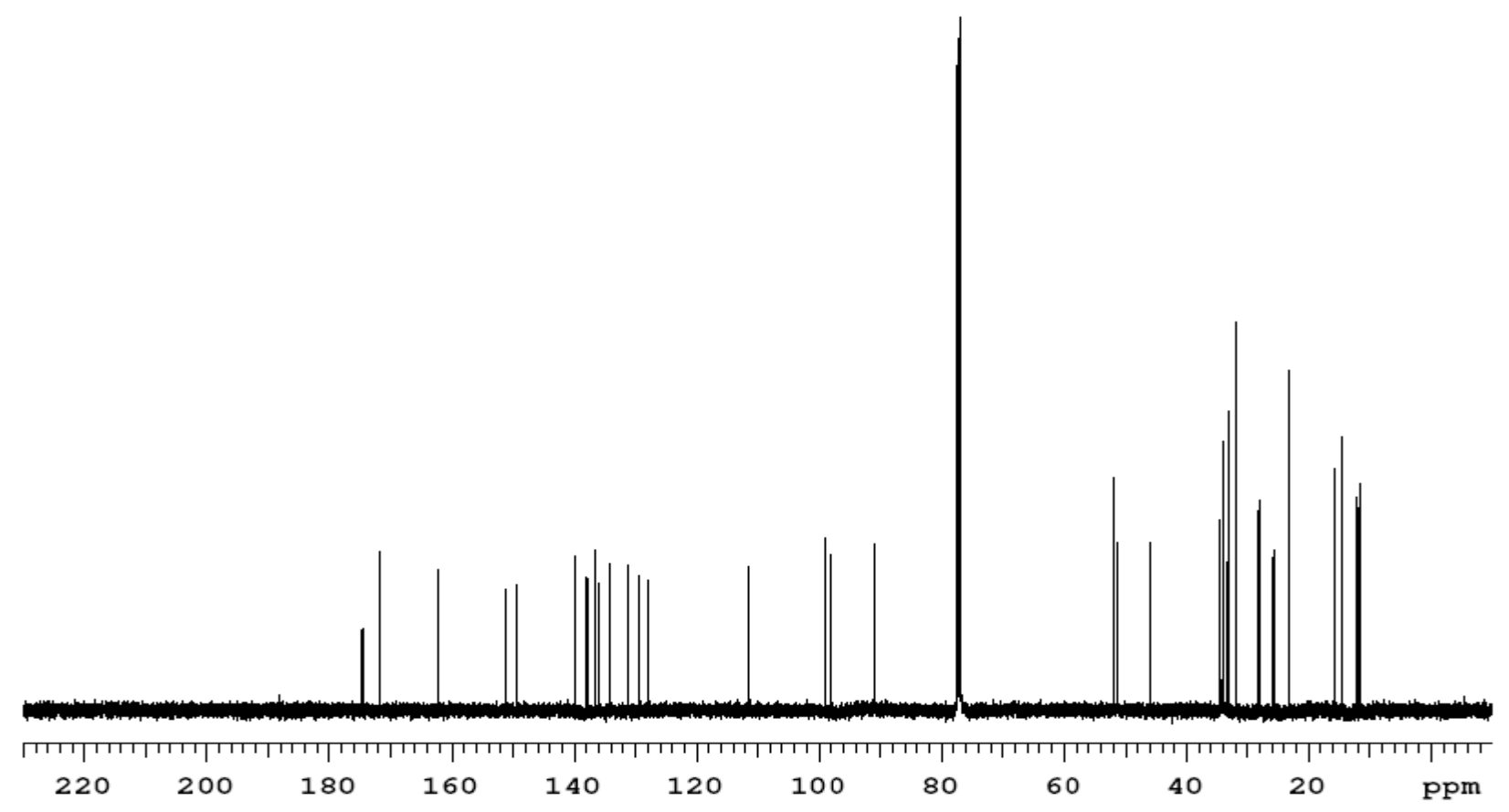

S59 
wgo_1H_IV145
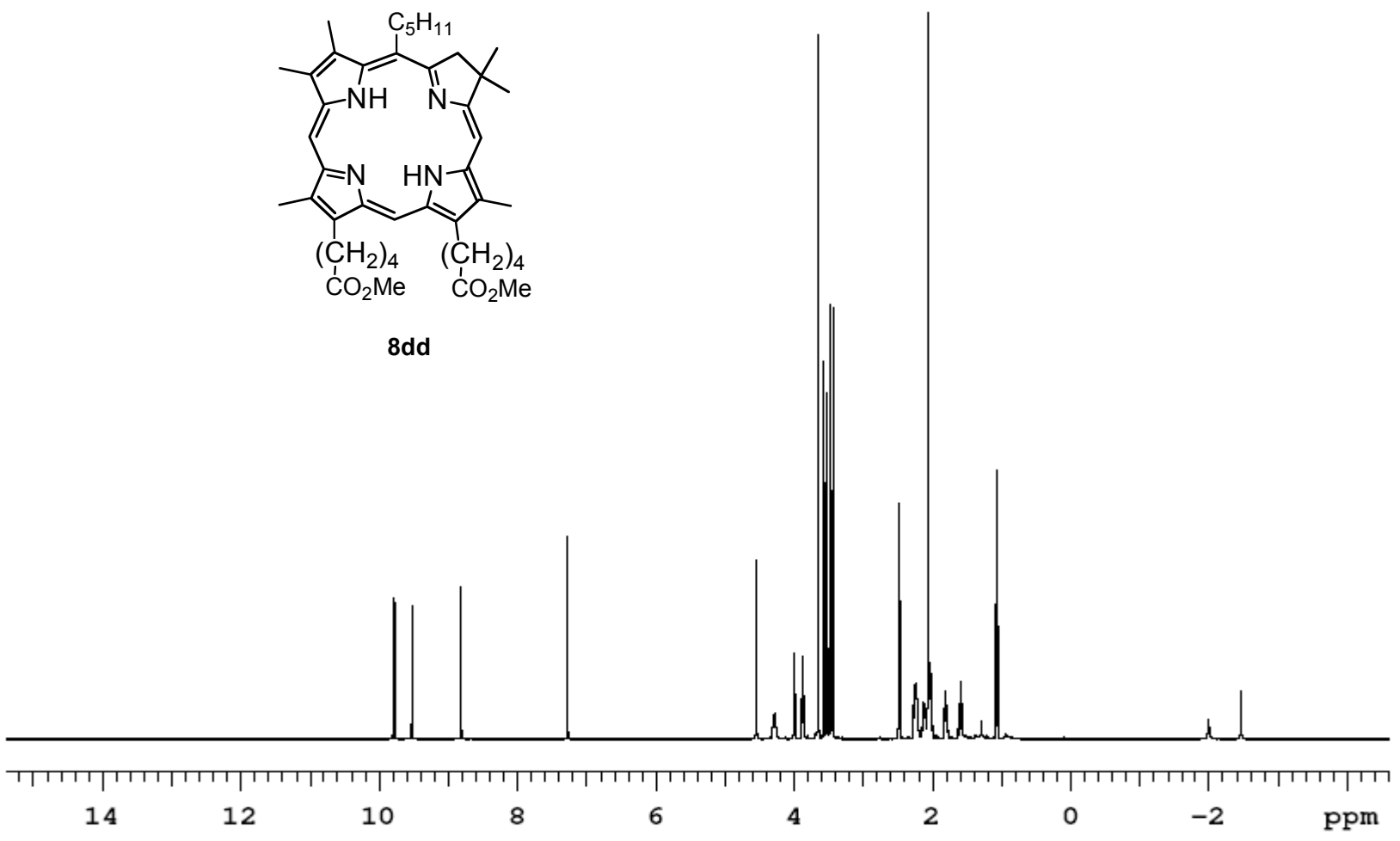

wgo_13C_IV145

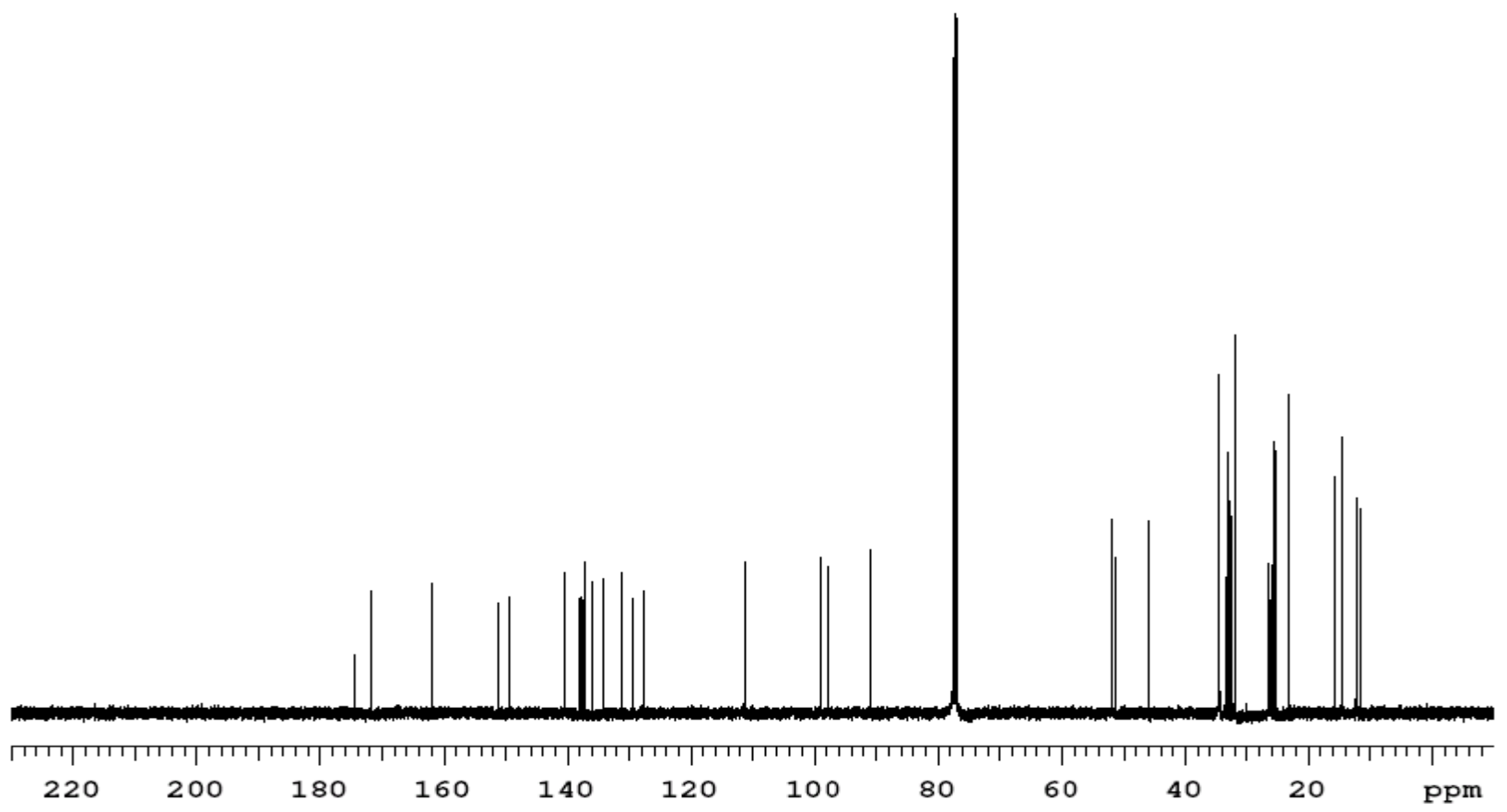

S60 
wgo_1H_IV146

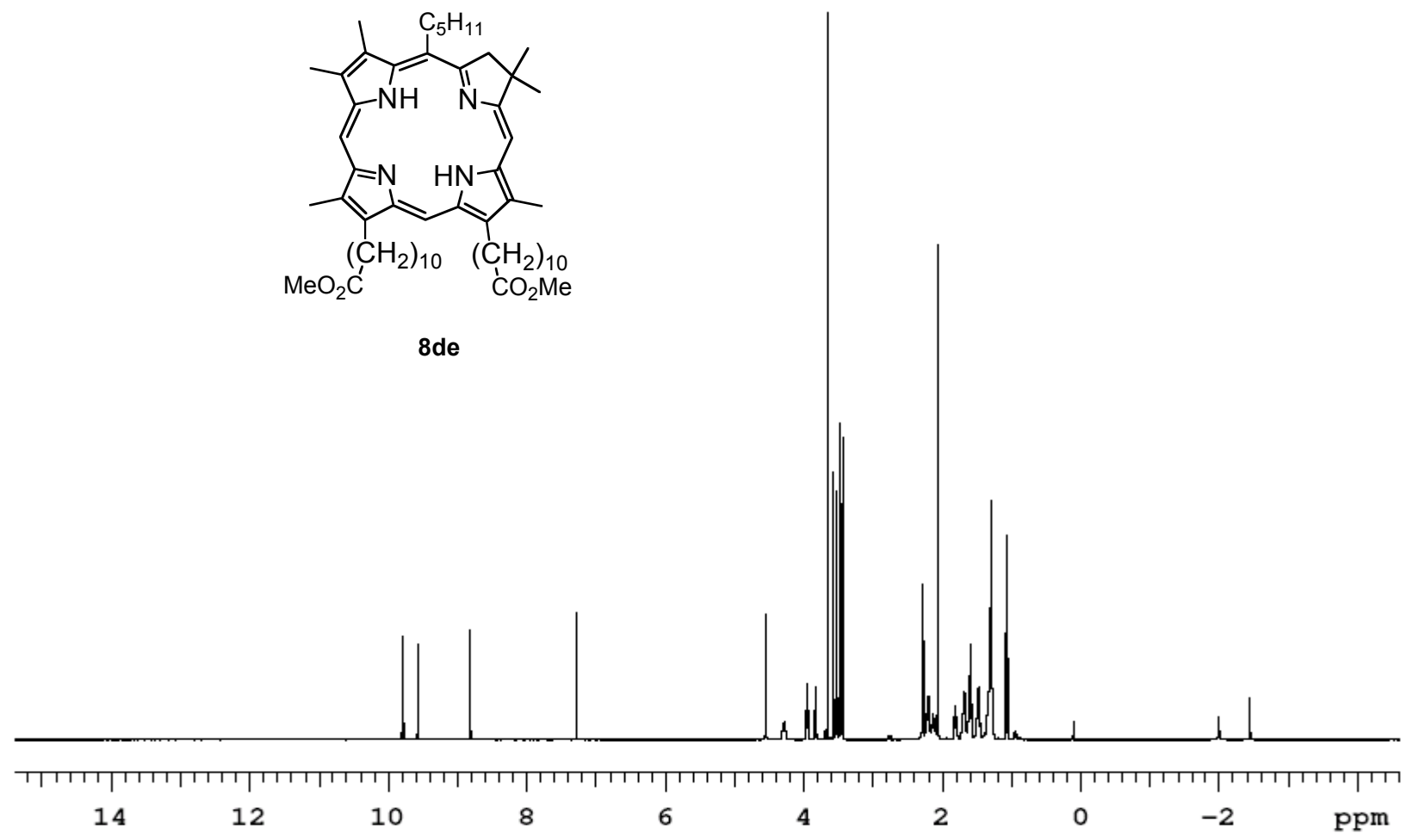

wgo_13C IV146

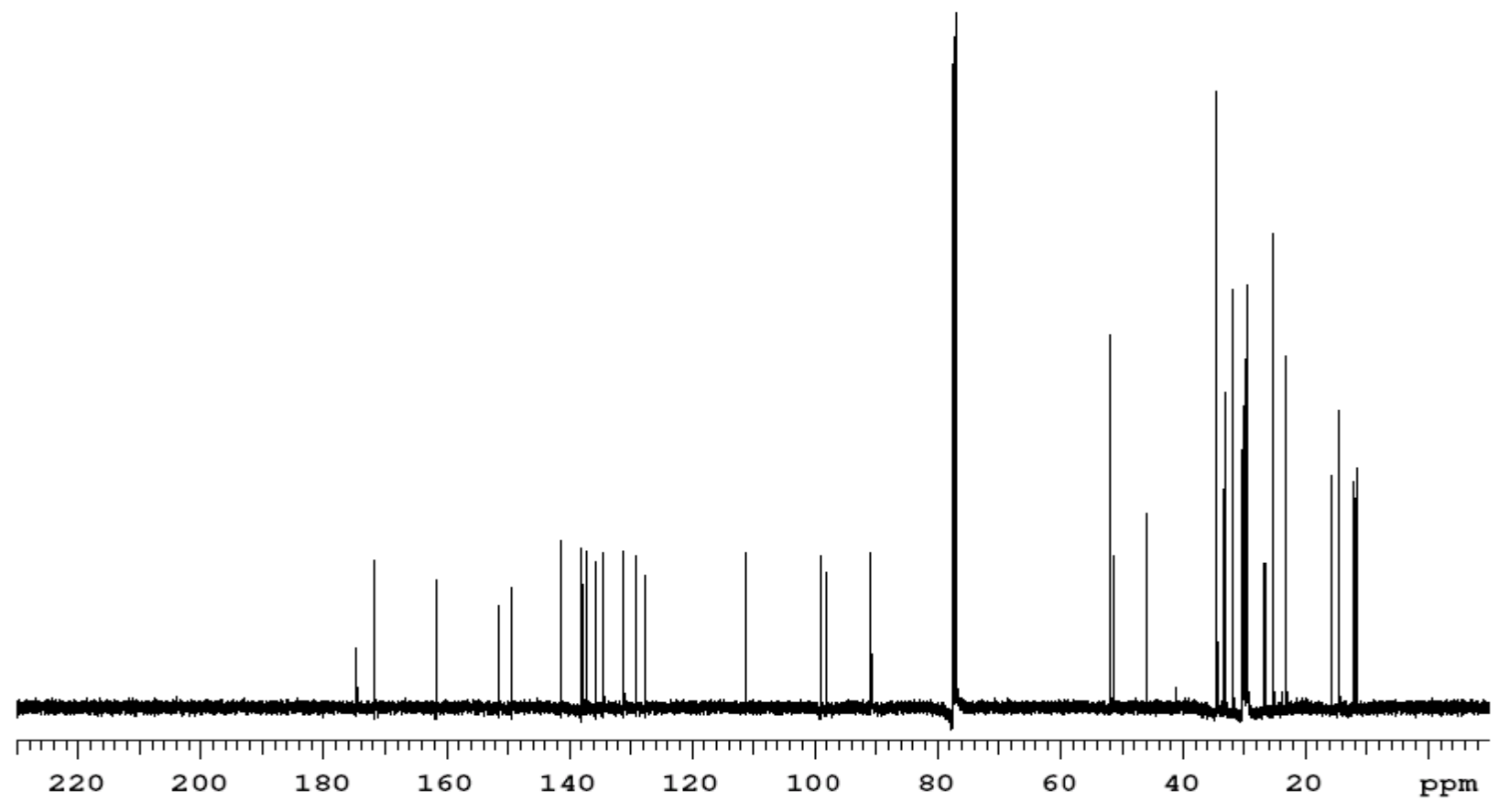


wr5p209

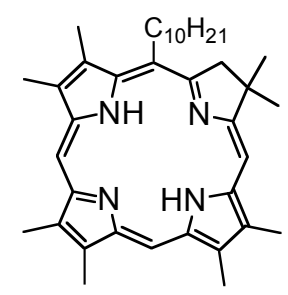

$8 e a$

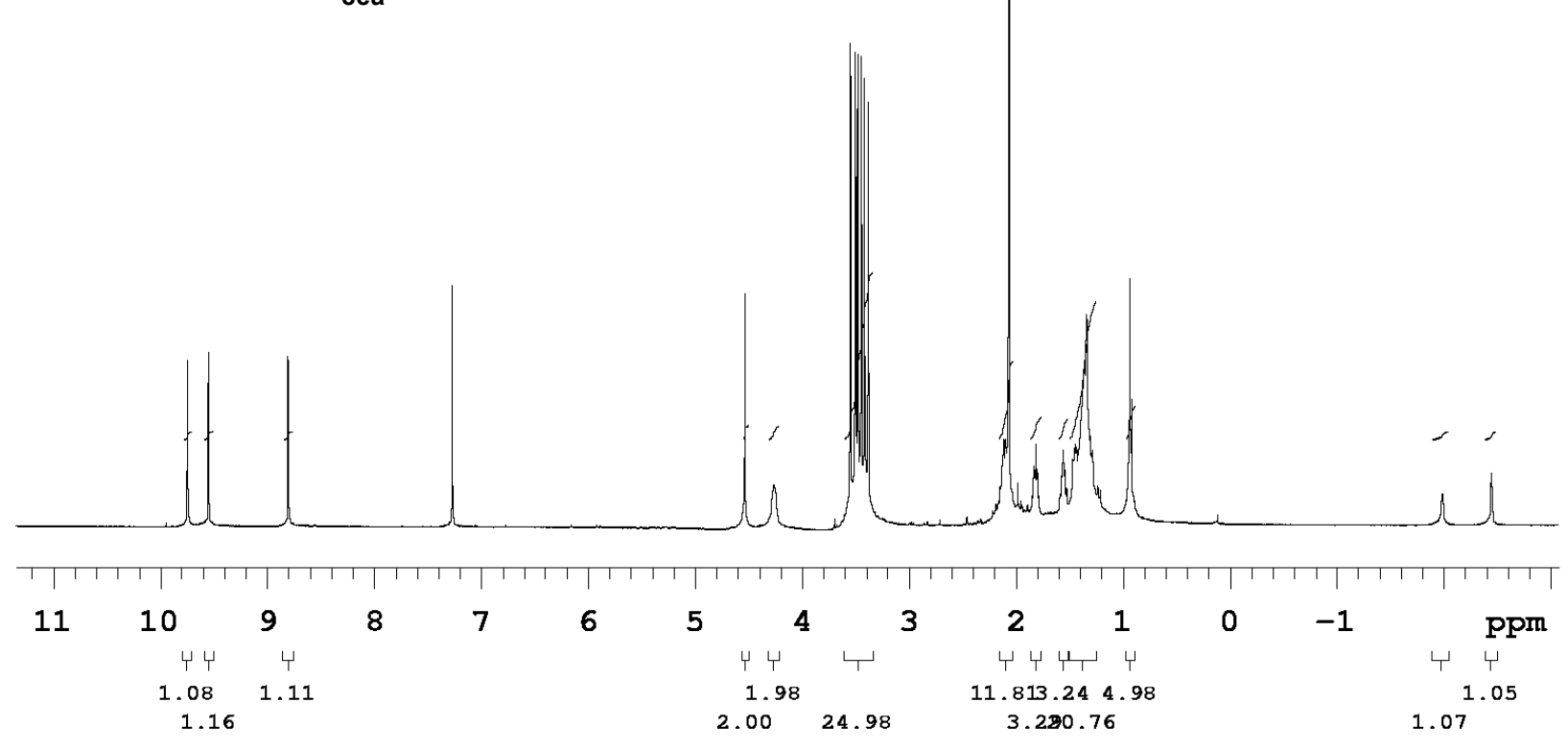

wr5p209C13

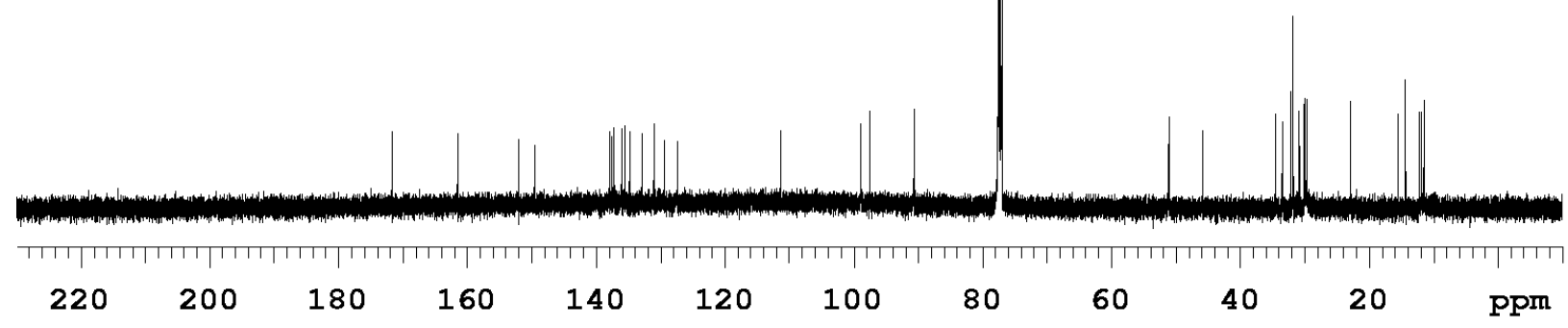


wr5p205
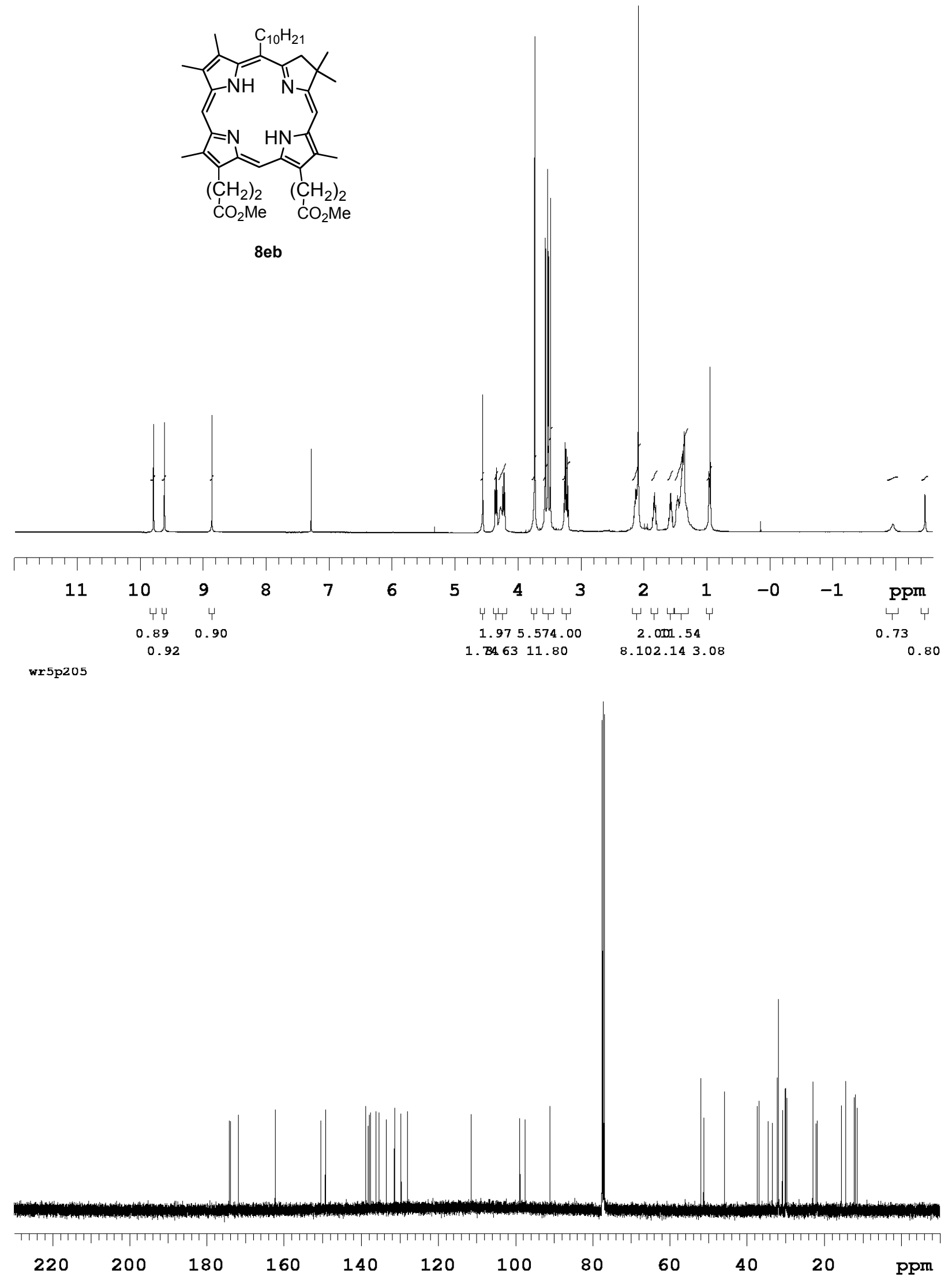
wgo_1H_IV149

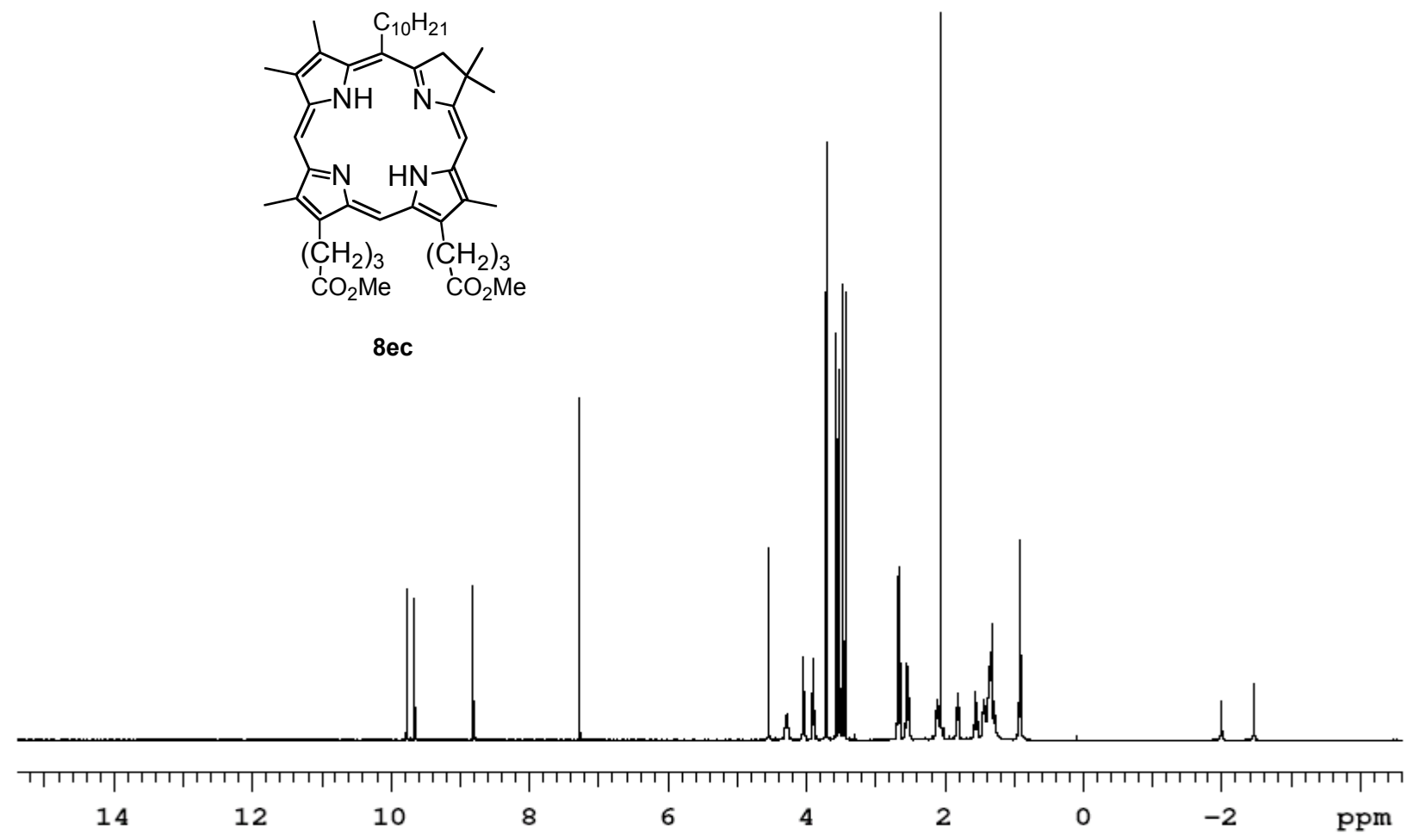

wgo $13 \mathrm{C} \quad$ IV149

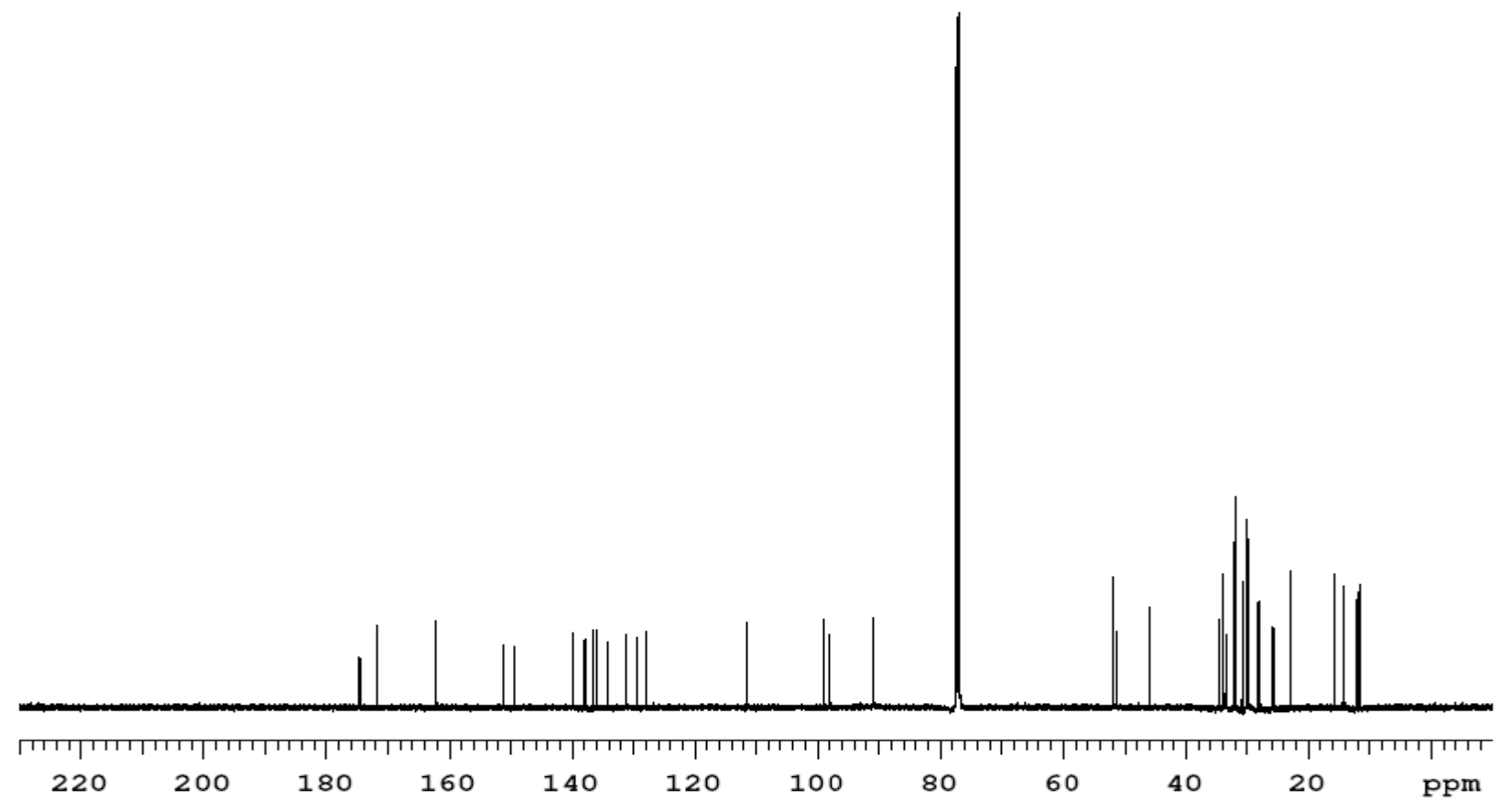


wgo_1H_IV150

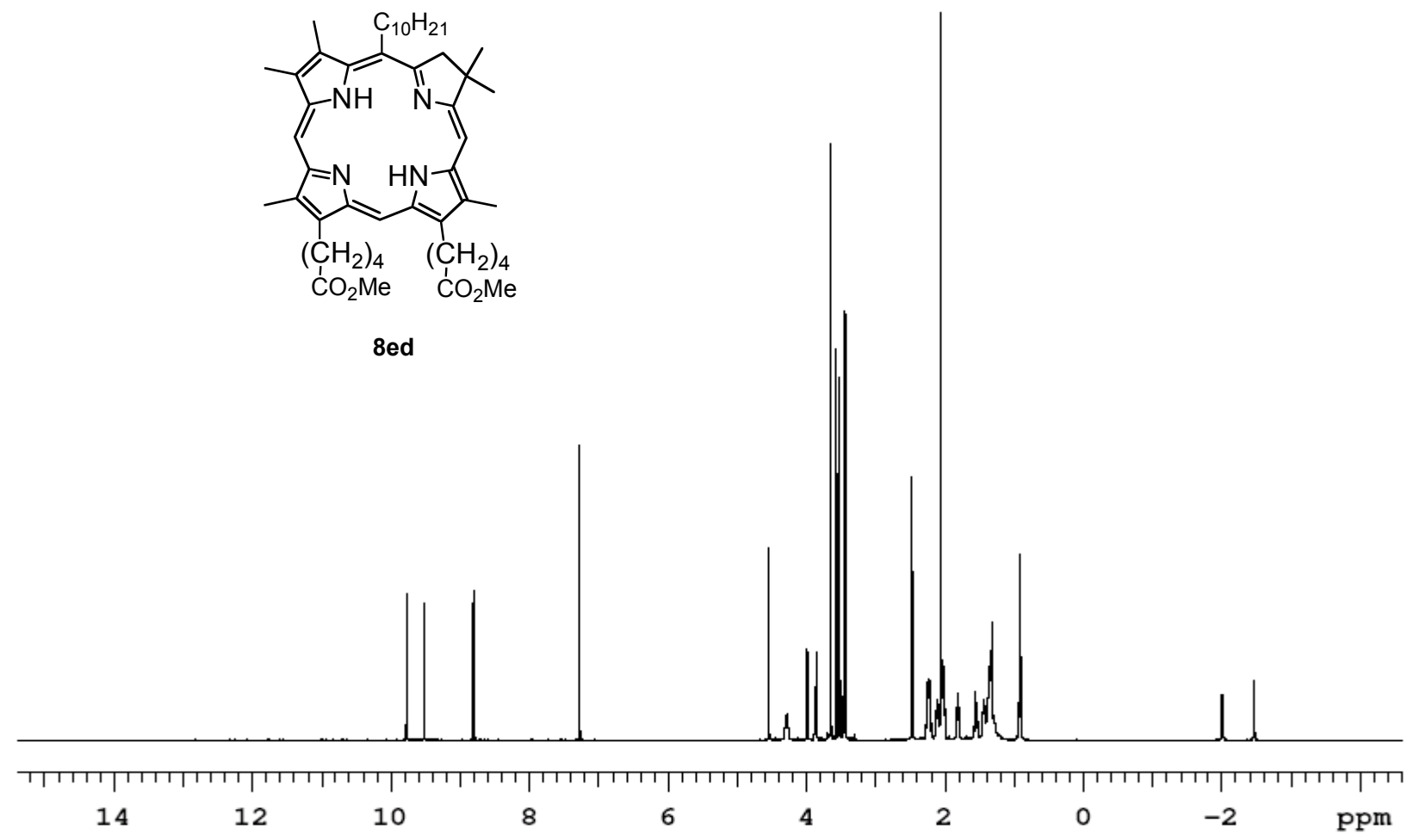

wgo 13C IV150

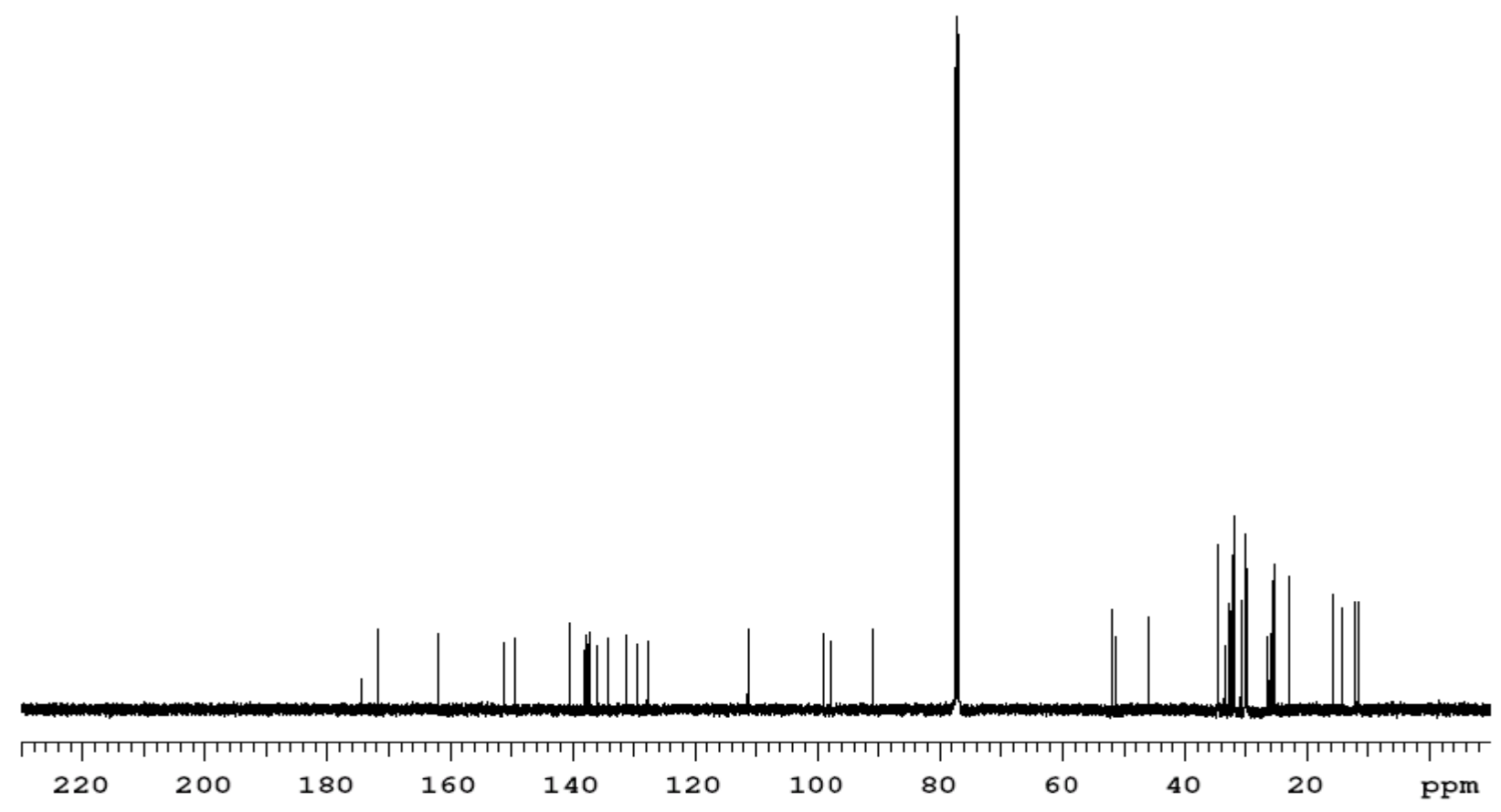


wgo_1H_IV151

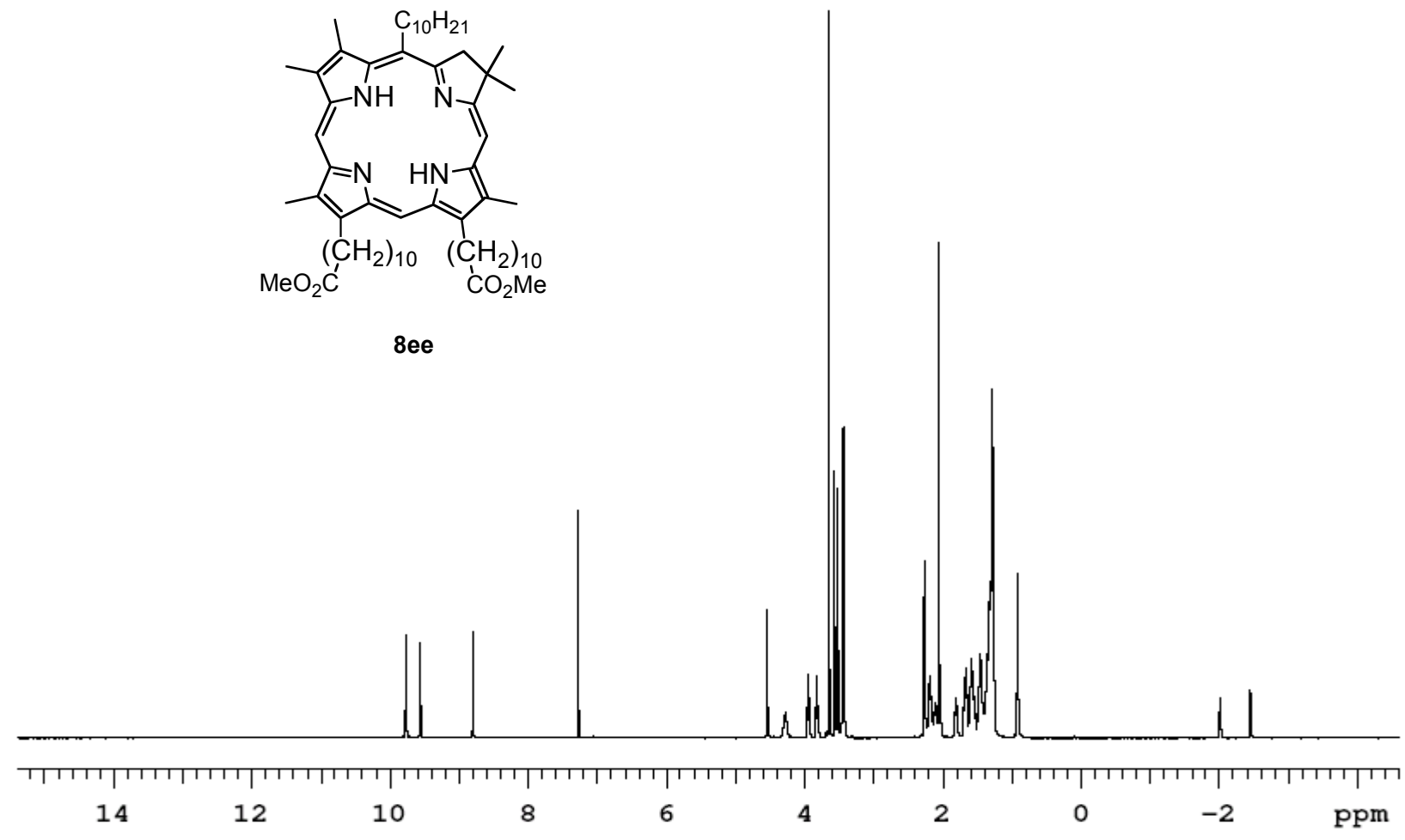

wgo_13C IV151

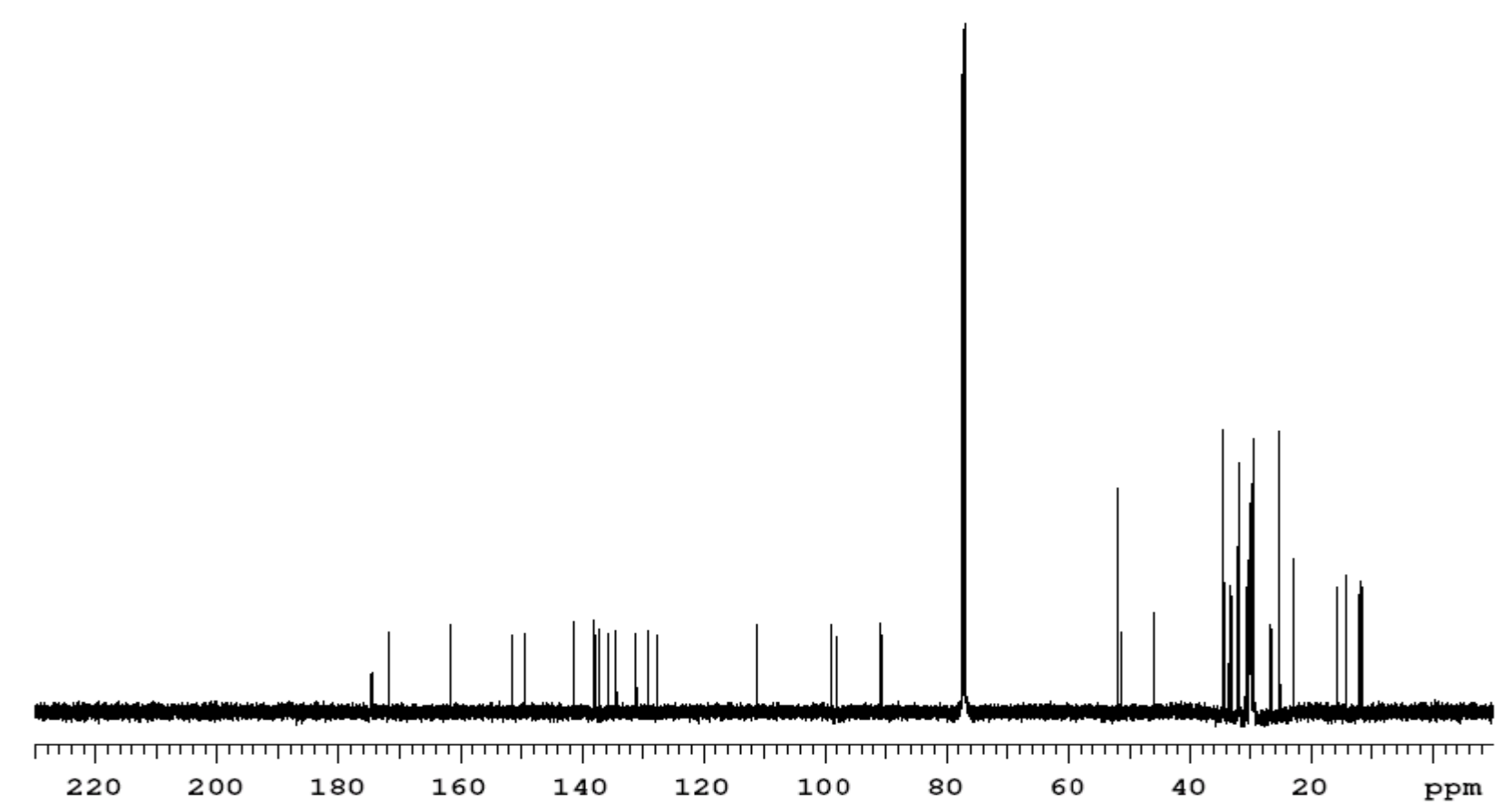




\section{Data collection}

A crystal (approximate dimensions $0.22 \times 0.20 \times 0.15 \mathrm{~mm}^{3}$ ) was placed onto the tip of a $0.1 \mathrm{~mm}$ diameter glass capillary and mounted on a CCD area detector diffractometer for a data collection at 173(2) K. A preliminary set of cell constants was calculated from reflections harvested from three sets of 20 frames. These initial sets of frames were oriented such that orthogonal wedges of reciprocal space were surveyed. This produced initial orientation matrices determined from 96 reflections. The data collection was carried out using MoKa radiation (graphite monochromator) with a frame time of 20 seconds and a detector distance of $4.9 \mathrm{~cm}$. A randomly oriented region of reciprocal space was surveyed to the extent of one sphere and to a resolution of $0.84 \AA$. Four major sections of frames were collected with $0.30^{\circ}$ steps in $\omega$ at four different $\phi$ settings and a detector position of $-28^{\circ}$ in $2 \theta$. The intensity data were corrected for absorption and decay (SADABS). ${ }^{1}$ Final cell constants were calculated from 3411 strong reflections from the actual data collection after integration (SAINT). ${ }^{2}$ Please refer to Table 1 for additional crystal and refinement information.

\section{Structure solution and refinement}

The structure was solved using Bruker SHELXTL ${ }^{3}$ and refined using Bruker SHELXTL. ${ }^{3}$ The space group C2/c was determined based on systematic absences and intensity statistics. A direct-methods solution was calculated which provided most non-hydrogen atoms from the E-map. Full-matrix least squares / difference Fourier cycles were performed which located the remaining non-hydrogen atoms. All non-hydrogen atoms were refined with anisotropic displacement parameters. All hydrogen atoms were placed in ideal positions and refined as riding atoms with relative isotropic displacement parameters. The final full matrix least squares refinement converged to $R 1=0.0536$ and $w R 2$ $=0.1441\left(F^{2}\right.$, all data $)$.

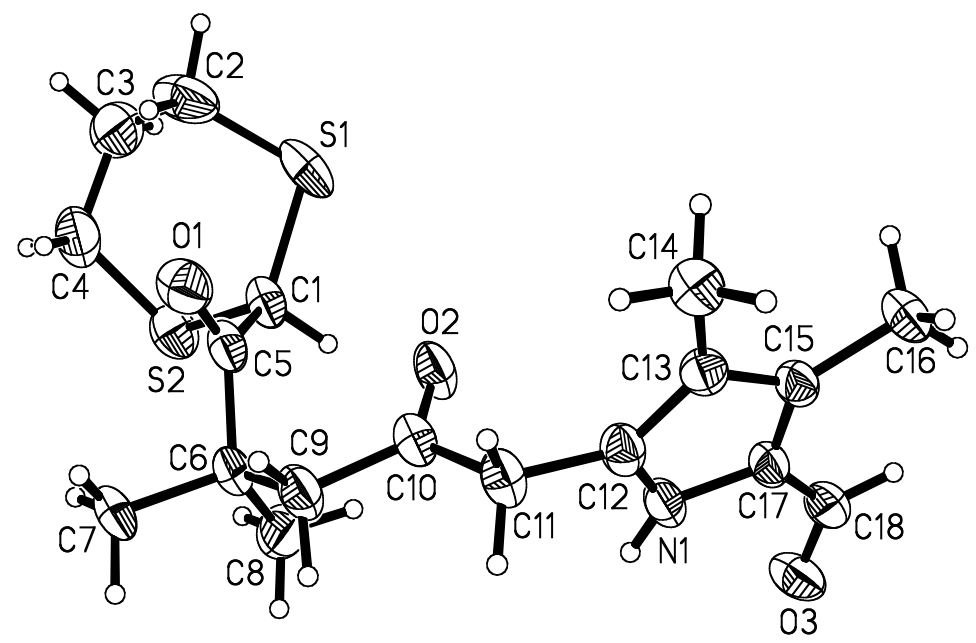

Figure 1. ORTEP representation of X-ray structure of $24 \mathrm{cB}$. 
Table 1. Crystal data and structure refinement for $\mathbf{2 4 c B}$.

\begin{tabular}{|c|c|c|}
\hline Identification code & \multicolumn{2}{|l|}{$04058 \mathrm{a}$} \\
\hline Empirical formula & \multicolumn{2}{|l|}{$\mathrm{C}_{18} \mathrm{H}_{25} \mathrm{~N} \mathrm{O}_{3} \mathrm{~S}_{2}$} \\
\hline Formula weight & \multicolumn{2}{|l|}{367.51} \\
\hline Temperature & \multicolumn{2}{|l|}{$173(2) \mathrm{K}$} \\
\hline Wavelength & \multicolumn{2}{|l|}{$0.71073 \AA$} \\
\hline Crystal system & \multicolumn{2}{|l|}{ Monoclinic } \\
\hline Space group & \multicolumn{2}{|l|}{$\mathrm{C} 2 / \mathrm{c}$} \\
\hline \multirow[t]{3}{*}{ Unit cell dimensions } & $a=35.397(3) \AA$ & $\alpha=90^{\circ}$ \\
\hline & $b=8.1002(7) \AA$ & $\beta=93.618(2)^{\circ}$ \\
\hline & $c=13.1578(12) \AA$ & $\gamma=90^{\circ}$ \\
\hline Volume & \multicolumn{2}{|l|}{$3765.1(6) \AA^{3}$} \\
\hline$Z$ & \multicolumn{2}{|l|}{8} \\
\hline Density (calculated) & \multicolumn{2}{|l|}{$1.297 \mathrm{Mg} / \mathrm{m}^{3}$} \\
\hline Absorption coefficient & \multicolumn{2}{|l|}{$0.298 \mathrm{~mm}^{-1}$} \\
\hline$F(000)$ & \multicolumn{2}{|l|}{1568} \\
\hline Crystal color, morphology & \multicolumn{2}{|l|}{ Yellow, Block } \\
\hline Crystal size & \multicolumn{2}{|c|}{$0.22 \times 0.20 \times 0.15 \mathrm{~mm}^{3}$} \\
\hline Theta range for data collection & \multicolumn{2}{|l|}{2.31 to $25.07^{\circ}$} \\
\hline Index ranges & \multicolumn{2}{|c|}{$-42 \leq h \leq 42,0 \leq k \leq 9,0 \leq l \leq 15$} \\
\hline Reflections collected & \multicolumn{2}{|l|}{18775} \\
\hline Independent reflections & \multicolumn{2}{|c|}{$3341[R(\mathrm{int})=0.0543]$} \\
\hline Observed reflections & \multicolumn{2}{|l|}{2646} \\
\hline Completeness to theta $=25.07^{\circ}$ & \multicolumn{2}{|l|}{$99.8 \%$} \\
\hline Absorption correction & \multicolumn{2}{|l|}{ Multi-scan } \\
\hline Max. and min. transmission & \multicolumn{2}{|l|}{0.9566 and 0.9373} \\
\hline Refinement method & \multicolumn{2}{|c|}{ Full-matrix least-squares on $F^{2}$} \\
\hline Data / restraints / parameters & \multicolumn{2}{|l|}{$3341 / 0 / 221$} \\
\hline Goodness-of-fit on $F^{2}$ & \multicolumn{2}{|l|}{1.110} \\
\hline Final $R$ indices $[I>2 \operatorname{sigma}(I)]$ & \multicolumn{2}{|c|}{$R 1=0.0536, w R 2=0.1351$} \\
\hline$R$ indices (all data) & \multicolumn{2}{|c|}{$R 1=0.0686, w R 2=0.1441$} \\
\hline Largest diff. peak and hole & \multicolumn{2}{|c|}{0.489 and -0.259 e. $\AA^{-3}$} \\
\hline
\end{tabular}


Notes:

1 An empirical correction for absorption anisotropy, R. Blessing, Acta Cryst. A51, 33-38 (1995).

2 SAINT V6.2, Bruker Analytical X-Ray Systems, Madison, WI (2001).

3 SHELXTL V6.10, Bruker Analytical X-Ray Systems, Madison, WI (2000).

4 A. Altomare, M. C. Burla, M. Camalli, G. Cascarano, C. Giacovazzo, A. Guagliardi, A. G. G. Moliterni, G. Polidori, R. Spagna. Sir97: a new tool for crystal structure determination and refinement. J. Appl. Cryst. 32, 115-119 (1998).

5 M. C. Burla, M. Camalli, B. Carrozzini, G. L. Cascarano, C. Giacovazzo, G. Polidori, R. Spagna. Sir2002: a new Direct Methods program for automatic solution and refinement of crystal structures. J. Appl. Cryst. (2003), in preparation.

6 A. L. Spek, Acta. Cryst. A46, C34 (1990). PLATON, A Multipurpose Crystallographic Tool, Utrecht University, Utrecht, The Netherlands, A. L. Spek (2000). 